INFRARED FACE RECOGNITION

A THESIS SUBMITTED TO

THE GRADUATE SCHOOL OF NATURAL AND APPLIED SCIENCES

OF

MIDDLE EAST TECHNICAL UNIVERSITY

BY

UĞUR KONUK

IN PARTIAL FULFILLMENT OF THE REQUIREMENTS

FOR

THE DEGREE OF MASTER OF SCIENCE

IN

ELECTRICAL AND ELECTRONICS ENGINEERING 

Approval of the thesis:

\section{INFRARED FACE RECOGNITION}

submitted by UĞUR KONUK in partial fulfillment of the requirements for the degree of Master of Science in Electrical and Electronics Engineering Department, Middle East Technical University by,

Prof. Dr. M. Gülbin Dural Ünver

Dean, Graduate School of Natural and Applied Sciences

Prof. Dr. Gönül Turhan Sayan

Head of Department, Electrical and Electronics Eng.

Prof. Dr. Gözde Bozdağı Akar

Supervisor, Electrical and Electronics Eng. Dept., METU

\section{Examining Committee Members:}

Prof. Dr. Uğur Halıcı

Electrical and Electronics Eng. Dept., METU

Prof. Dr. Gözde Bozdağı Akar

Electrical and Electronics Eng. Dept., METU

Assist. Prof. Dr. Sinan Kalkan

Computer Eng. Dept., METU

Assist. Prof. Dr. Sevinç Figen Öktem

Electrical and Electronics Eng. Dept., METU

Assist. Prof. Dr. Göksel Günlü

Electrical and Electronics Eng. Dept., TURGUT ÖZAL UNI.

Date: $\quad 07 / 09 / 2015$ 
I hereby declare that all information in this document has been obtained and presented in accordance with academic rules and ethical conduct. I also declare that, as required by these rules and conduct, I have fully cited and referenced all material and results that are not original to this work.

Name, Last name : Uğur KONUK

Signature 


\title{
ABSTRACT
}

INFRARED FACE RECOGNITION

\author{
Konuk, Uğur \\ M.S., Department of Electrical and Electronics Engineering \\ Supervisor: Prof. Dr. Gözde Bozdağı Akar
}

September 2015, 95 pages

Face recognition is a leading biometrics technique that fulfills the increasing need to identify a person in today's world. Face recognition also has broad range of utilization, such as commercial and law enforcement applications. That is the reason why it still gathers a lot of attention and is an active research topic. Nevertheless visible spectrum face recognition algorithms are not free of challenges. Illumination, pose, expression variances and existence of facial disguises still degrade the performance of recognition systems that utilize visible spectrum face images. That fact paved the way for infrared face recognition to emerge as an alternative solution to those limitations. In this thesis work, first a review of the infrared face recognition algorithms is presented. Four different methods, one of which is proposed for the first time in this thesis work, are implemented and compared in terms of recognition performance. The proposed method investigates the utilization of vascular network method with blood perfusion transform of the thermal data. Experiments are performed on Terravic and IRIS database.

Keywords: IR face recognition, Blood perfusion, vascular network, eigenface, Hausdorff distance, IRIS database, Terravic database 


\title{
$\ddot{0} \mathbf{z}$
}

\section{KIZILÖTESİ YÜZ TANIMA}

\author{
Konuk, Uğur \\ Yüksek Lisans, Elektrik Elektronik Mühendisliği Bölümü \\ Tez Yöneticisi: Prof. Dr. Gözde Bozdağı Akar \\ Eylül 2015, 95 sayfa
}

Yüz tanıma, günümüzde artan bir insanın kimliğini doğrulama ihtiyacını karşılayan öncü bir biometrik tekniktir. Yüz tanıma ticari ve güvenlik uygulamalarında geniş bir kullanım alanına da sahiptir. Bu sebeple yüz tanıma çok ilgi gören aktif bir araştırma konusudur; fakat görünür bantta yüz tanıma çalışmalarında da karşılaşılan problemler mevcuttur. Işık, poz ve ifade değişimleri ile yüz üzerinde kılık değiştirme mevcudiyeti görünür 1şıkta yüz tanıma sistemlerinin performansını düşürmektedir. Bu durum, yaşanan sorunlara alternatif bir çözüm olarak kızılötesi yüz tanımanın öne çıkmasına yol açmıştır. Bu tez çalışmasında ilk olarak kızılötesi yüz tanıma tekniklerinin genel bir incelemesi yapılmıştır. Biri ilk kez bu tez çalışması kapsamında önerilen dört farklı teknik gerçeklenmiş ve performansları karşılaştırılmıştır. Önerilen teknik, vasküler ağ metodunun kan perfüzyonu verisine uygulanmasını inceler. Denemeler Terravic ve IRIS veri tabanlarında gerçekleştirilmiştir.

Anahtar Kelimeler: Kızılötesi yüz tanıma, kan perfüzyonu, vasküler ağ, eigen yüz, Hausdorff mesafesi, IRIS veritabanı, Terravic veritabanı 
To my parents, my brother and Yağmur 


\section{ACKNOWLEDGMENTS}

I would like to thank Prof. Dr. Gözde Bozdağı Akar for her valuable supervision, support and tolerance throughout the development and improvement of this thesis.

I am grateful to Melis Eryılmaz for her support throughout the development and the improvement of this thesis. I am also grateful to Aselsan Electronics Industries Inc. for the resources and facilities that I use throughout thesis.

Thanks a lot to all my friends for their great encouragement and their valuable help to accomplish this work. Especially I would like to thank Cumhur Çakmak, Elif Sarıtaş and Murat Kumru for their help.

Finally, I would like to thank my beloved ones. I would like to thank my parents for bringing up and trusting in me and for all the love, which is mutual, and support that they showed throughout the years. I would like to thank my brother, whom I love in deep, for being the best brother and friend that one can imagine. Words may not describe his value to me. I would like to also thank his wife Irem for being the sister that I did not have before. Last but far from least I would like to thank my girlfriend Yağmur, whom I adore, for making my every single day more beautiful and for being the joy of my life. Her love and support is invaluable to me. 


\section{TABLE OF CONTENTS}

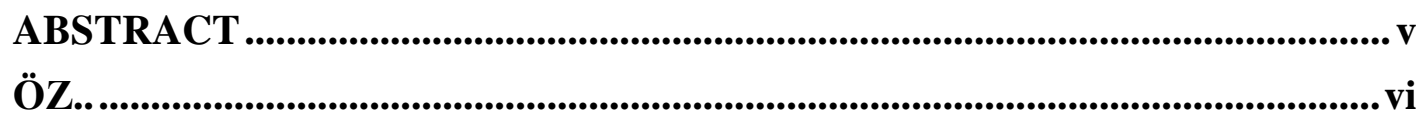

ACKNOWLEDGMENTS ..................................................................................iii

TABLE OF CONTENTS...................................................................................

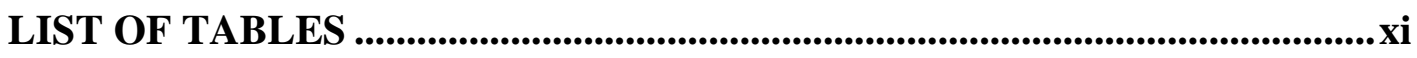

LIST OF FIGURES ...........................................................................................

LIST OF ABBREVIATIONS ........................................................................ CHAPTERS

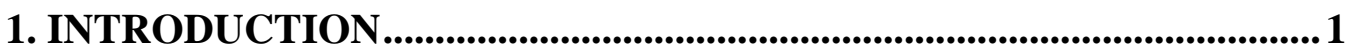

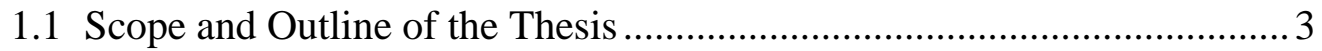

2. LITERATURE REVIEW ............................................................................5

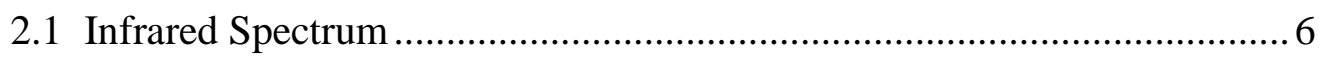

2.2 Advantages and Limitations of Infrared Face Recognition ...................... 7

2.2.1 Advantages of Infrared Face Recognition....................................... 7

2.2.2 Limitations of Infrared Face Recognition ....................................... 9

2.3 Infrared Face Recognition Techniques................................................ 11

2.3.1 Holistic Approaches ..................................................................... 11

2.3.2 Feature Based Approaches .............................................................. 14

2.3.3 Multimodal and Intermodal Techniques ........................................ 17

2.3.3.1 Multimodal Techniques .......................................................... 18

2.3.3.1.1 Decision Based Fusion........................................................... 18

2.3.3.1.2 Data Based Fusion ............................................................... 19

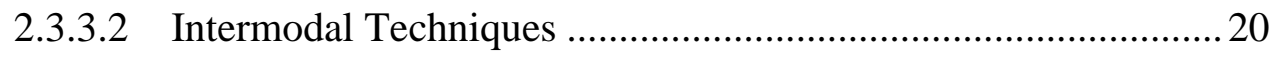

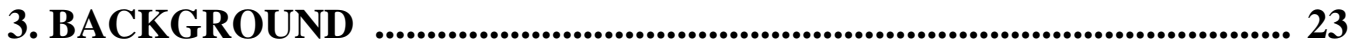

3.1 Face Segmentation Algorithm Employed in Implemented Recognition

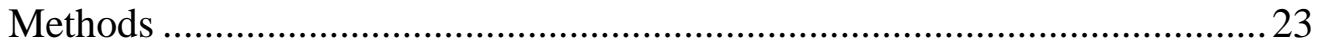

3.2 Background Information on Implemented Methods …......................... 29

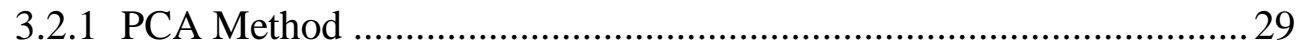

3.2.2 Blood Perfusion Method ................................................................. 33 
3.2.3 Vascular Network Method .............................................................40

4. ALGORITHMS FOR INFRARED FACE RECOGNITION ..................49

4.1 PCA Method Implementation .................................................................49

4.2 PCA Method Applied with Blood Perfusion Transform .........................51

4.3 Vascular Network Method Implementation .........................................52

4.4 Vascular Network Method Applied to Blood Perfusion Transform of the

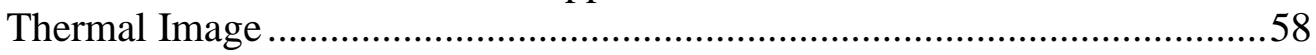

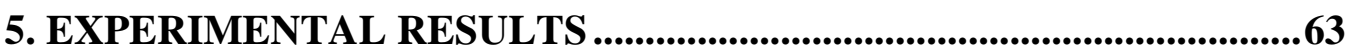

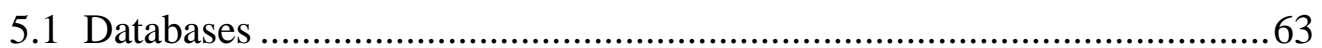

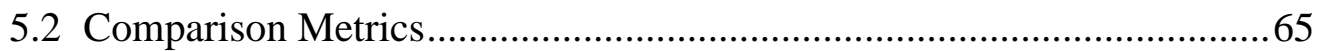

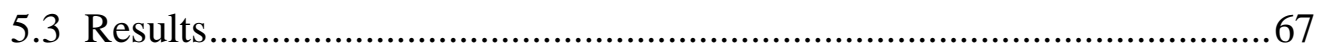

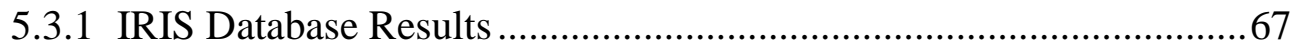

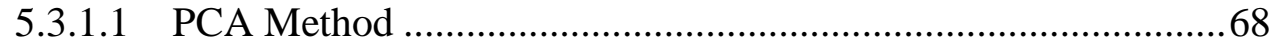

5.3.1.2 PCA Method with Blood Perfusion Transform .........................69

5.3.1.3 Vascular Network Method ......................................................... 72

5.3.1.4 Vascular Network Method Applied to Blood Perfusion Transform of the Thermal Data ............................................................................ 74

5.3.2 Terravic Database Results ..............................................................77

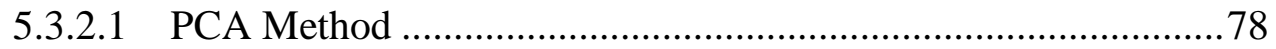

5.3.2.2 PCA Method with Blood Perfusion Transform ......................... 78

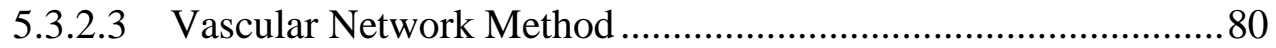

5.3.2.4 Vascular Network Method Applied to Blood Perfusion Transform

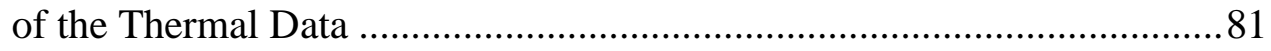

6. CONCLUSION AND FUTURE WORK..................................................85

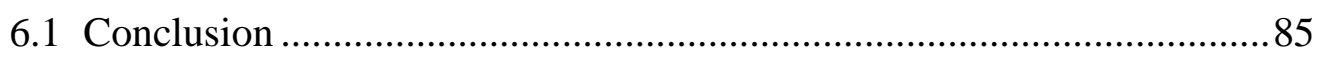

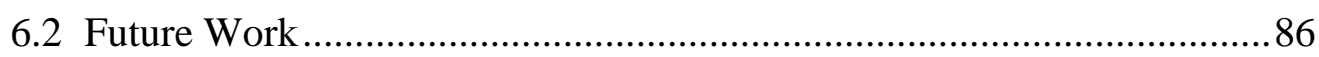

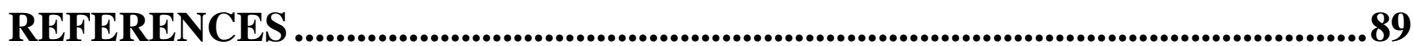




\section{LIST OF TABLES}

\section{TABLES}

Table 3-1 Terms in blood perfusion transform and their descriptions..................... 35

Table 5-1 Definitions of true positive, true negative, false positive and false negative 66

Table 5-2 : Results obtained for the implemented methods in IRIS Database ......... 68

Table 5-3 : Results obtained for the implemented methods in Terravic Database ... 77 


\section{LIST OF FIGURES}

\section{FIGURES}

Figure 2.1 Human body Planck distribution [10] ............................................

Figure 2.2 Histogram of the LWIR images under luminance variance...................... 8

Figure 2.3 Images taken under different ambient temperatures [21]. Ambient temperatures are, from left to right, first row 28.4 and $28.7^{\circ} \mathrm{C}$; second row 28.9 and $29.3{ }^{\circ} \mathrm{C}$.

Figure 2.4 Photographs taken at different time intervals after alcohol intake [21]...11

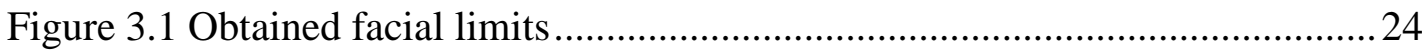

Figure 3.2 Curve fitting procedure applied to the cropped image ...........................26

Figure 3.3 Segmented face with the explained method........................................2

Figure 3.4 Original image from IRIS database and obtained mask with the utilization

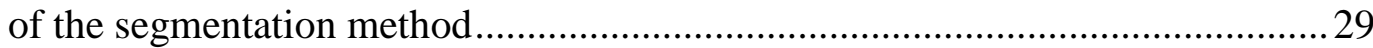

Figure 3.5 A simple flowchart for implementing PCA for face recognition ............ 30

Figure 3.6 Blood perfusion vs temperature graph ............................................... 37

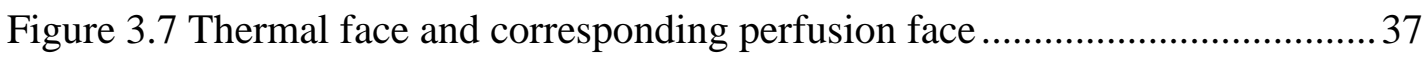

Figure 3.8 General flowchart of the methods that utilize perfusion transform ..........38

Figure 3.9 Different perfusion models are presented for visual comparison [77]. The image on the left is the original thermal image. The image in the middle is the original blood perfusion image. The image on the right is Pennes blood perfusion image. 40

Figure 3.10 The effect of anisotropic diffusion. The top left image is a thermal wrist image and top right image is the corresponding line profile shown as a black line. The bottom left image is the resulting image after anisotropic diffusion and the bottom right image is its corresponding line profile. [69] .42 
Figure 3.11 Original segmented image and extracted blood vessels with top hat operation.

Figure 3.12 Flowchart for the improved algorithm proposed by Buddharaju and Pavlidis [71] .45

Figure 4.1 Flowchart of the PCA method described in the thesis work 50

Figure 4.2 Flowchart of PCA method applied with blood perfusion transform .......51

Figure 4.3 Flowchart of the vascular network method feature vector approach A... 53

Figure 4.4 Flowchart of the vascular network method feature vector approach B ... 53

Figure 4.5 Flowchart of the vascular network method with proposed feature vector

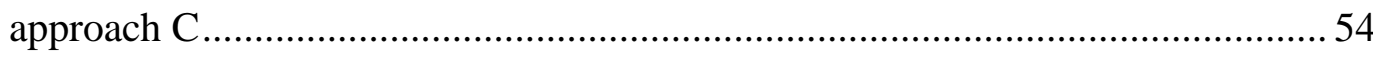

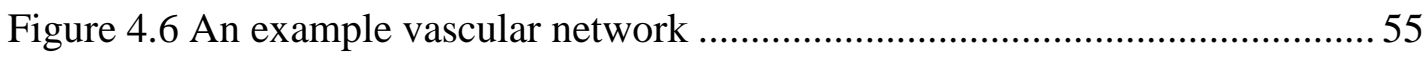

Figure 4.7 Flowchart of the proposed method feature vector approach A................58

Figure 4.8 Flowchart of the proposed method feature vector approach B...............59

Figure 4.9 Flowchart of the proposed method feature vector approach C...............59

Figure 4.10 Vascular networks obtained from the same person utilizing thermal image (left) and blood perfusion transform of the thermal image (right) ............ 61

Figure 5.1 Various challenging conditions for images in IRIS database ...................64 64

Figure 5.2 One image from each database are given side by side (Terravic image on

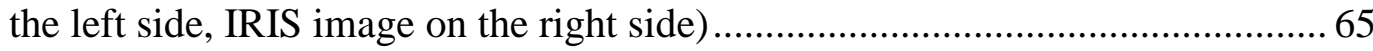

Figure 5.3 Original image and blood perfusion image ........................................ 70

Figure 5.4 Thermal image and perfusion image ................................................. 70

Figure 5.5 Thermal image and perfusion image .................................................. 70

Figure 5.6 Steps of the algorithm and obtained vascular network.......................... 74

Figure 5.7 Steps of the proposed method and obtained vascular network................ 76

Figure 5.8 Thermal image and corresponding perfusion image ............................. 79

Figure 5.9 Thermal image and corresponding perfusion image ............................. 79

Figure 5.10 Steps of the vascular network method and obtained vascular network. 80 Figure 5.11 An image from the Terravic database, steps of the proposed method and

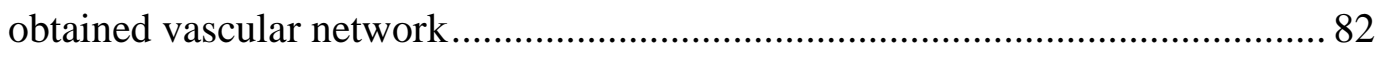




\title{
LIST OF ABBREVIATIONS
}

\author{
AAM : Active Appearance Model \\ ICA : Independent Component Analysis \\ FAR : False Acceptance Rate \\ FRR : False Rejection Rate \\ LDA : Linear Discriminant Analysis \\ LWIR : Long Wave Infrared \\ MHD : Modified Hausdorff Distance \\ MWIR : Medium Wave Infrared \\ NIR : Near Infrared \\ PCA : Principal Component Analysis \\ RROI : Rectangular Region of Interest \\ SWIR : Short Wave Infrared
}




\section{CHAPTER 1}

\section{INTRODUCTION}

The need to identify or authenticate a person is increasing. Whether it is an online financial transaction, a simple payment in a retail store or to enter a place where a clearance level is needed, there is a need for reliable recognition or authentication mechanism. In order to recognize people, there exist different techniques. One of the most frequently and traditionally utilized technique is based on knowledge. This corresponds to passwords, PINs or private information like your mother's maiden name. Although this is a quite simple method, it has its own deficiencies. Longer and more complex passwords offer more security. However, people tend to choose short and simple passwords, as it will be easier for them to remember. This jeopardizes the security since those passwords are easier to be seen, guessed or cracked. Strong passwords are harder to remember so there is a risk involved there as well, that they could be forgotten. Another commonly utilized technique is based on possession, which corresponds to cards, tokens, and keys. In this method, the downside is that possessions can be stolen, corrupted or duplicated. The third method and the most promising one is the Biometrics. Biometrics is defined as the technologies utilized to identify or authenticate a person based on physiological or behavioral traits [1]. Biometrics is a more reliable method than the other two methods since traits assessed in Biometrics cannot be forgotten, stolen or duplicated.

Due to the above mentioned factors, the interest in biometrics has increased rapidly in the last ten years. The revenue for biometrics was \$20 million in 1996 [2] and \$7 
billion in 2012. It is projected to grow to $\$ 15$ billion by the year 2015 according to the Biometrics Research Group's report [3]. According to "Global Biometric Systems Market Forecast \& Opportunities, 2018” by TechSci Research the global biometrics revenue is projected to reach $\$ 20$ billion by 2018 [4].

Authentication is utilized to verify one's identity; that is, the biometric system tests whether the person that enrolls for authentication has the same biometric data of the claimed identity. As it is obvious from the definition, authentication process can have two outcomes; the person can be verified or rejected. Other than authentication, biometrics can also be utilized for identification. Identification aims to identify a person with the biometric data. The biometric data presented is compared with the previously enrolled biometric data belonging to all subjects to identify that person. This thesis work focuses on identification.

There exist many biometrics techniques in the literature. One of the most popular of those techniques is face recognition [5]. Unlike some other biometric techniques, such as iris, DNA, finger print or retina analysis, face recognition does not need cooperation with the subject. The advantages and disadvantages of popular biometrics techniques are investigated in the work of Philipp et al. [5], which is not the scope of this thesis work. Face recognition has discriminative power and face recognition algorithms achieve high recognition rates. It has been first tested for visible spectrum since the cost of visible spectrum cameras is inexpensive compared to other modalities such as infrared. Also the fact that humans see in the visible spectrum and is able to easily recognize other people may have contributed to favoring visible spectrum. Nevertheless visible spectrum face recognition algorithms are not free of limitations. One of the major challenges encountered in visible spectrum face recognition systems is illumination variance [6]. This paved the way for infrared face recognition to emerge as an alternative solution. Face recognition in infrared spectrum also has some other advantages compared to visible spectrum such as superior performance in presence of disguise, pose and expression variance. These advantages will be investigated in depth in the latter chapter. 


\subsection{Scope and Outline of the Thesis}

The scope of this thesis is to understand and evaluate algorithms used in infrared face recognition. Three methods which are representing different class of approaches are implemented. In addition a new method, which utilizes vascular network method with blood perfusion transform of the thermal data, is proposed. Experimental results obtained in IRIS database and Terravic database are presented. Obtained experimental results for each algorithm are compared with each other and with the existing works in literature in terms of recognition performance.

The outline of the thesis is as follows. In Chapter 2, infrared spectrum and its spectral bands are discussed first. Also in this chapter, the advantages and limitations of utilizing infrared images compared to visible spectrum images for face recognition are discussed. This chapter is concluded with the presentation of the state of the art infrared face recognition techniques.

In Chapter 3, four infrared face recognition algorithms and the literature regarding those algorithms are discussed in detail. In the beginning of the chapter, segmentation method that is commonly used in the investigated face recognition algorithms is discussed. Then the algorithms are presented in the following manner: First the significant works related to the algorithm are presented. Then the algorithm implemented in this thesis is explained.

The experimental results of the implemented algorithms are presented and compared in Chapter 4. First the experimental results obtained in IRIS database are presented. Then the results obtained in Terravic database are presented and compared to the results in IRIS database.

Finally in Chapter 5, a summary of the thesis work and possible future work is presented. 


\section{CHAPTER 2}

\section{LITERATURE REVIEW}

Face recognition, as mentioned in the former section, gathered a lot of interest from the scientific community as a biometrics technique.

Earlier approaches in face recognition concentrated in visible spectrum images. For face recognition purpose visible spectrum images are subject to a decrease in performance under illumination variance [7]. A possible solution to this problem is the use of 3D data and infrared spectrum images. 3D face images are inherently thought to be invariant under illumination. However, Bowyer et al. [8] stated that 3D images are also affected by variation in illumination and called the inherent idea that the $3 \mathrm{D}$ images are invariant to illumination variance a "myth". Also the cost, the processing speed are disadvantages of 3D data [9]. Infrared spectrum is another modality that can be employed to be secured from the performance degradation under luminance variance [10]. Infrared images can also be utilized when there is no light at all, unlike its visible counterpart.

In this chapter infrared spectrum, its advantages and limitations compared to visible spectrum and infrared face recognition techniques are discussed. First, the infrared spectrum and spectral bands of infrared spectrum are discussed. It is followed by the advantages and disadvantages of the infrared face recognition compared to visible spectrum face recognition. In the last part of this chapter an overview of the existing methods in infrared face recognition is represented. 


\subsection{Infrared Spectrum}

Infrared spectrum consists 4 main spectral bands. These spectral bands are near infrared (NIR, 0.7 to $1.4 \mu \mathrm{m}$ ), short wave infrared (SWIR, 1.4 to $3 \mu \mathrm{m}$ ), medium wave infrared (MWIR, 3 to $5 \mu \mathrm{m}$ ) and long wave infrared (LWIR, 8 to $15 \mu \mathrm{m}$ ) [10]. This categorization is based on the dramatic characteristic differences observed between these bands, such as contrast, background signal, aperture constraints, scene properties and atmospheric transmission under varying weather conditions. NIR and SWIR are called reflected infrared [11]. NIR band is "active" in nature [12]. Illuminators are needed for NIR which is a limitation for face recognition. Those illuminators are invisible to human eye so they can still remain unobtrusive [13]. MWIR and LWIR are naturally emitted by the human body. MWIR have higher contrast, better clear weather performance, higher transmissivity in high humidity and also higher resolution compared to LWIR. LWIR have higher signal to noise ratio, reduced background clutter, higher immunity to atmospheric turbulence and better performance in fog, dust and wind compared to MWIR. These differences are a result of optical equipment used in these bands. MWIR has smaller optical diffraction compared to LWIR.

The human body has a maximum Planck distribution at around $9 \mu \mathrm{m}$ which is in the range of LWIR [10]. This can be observed in Figure 2.1. Also the fact that LWIR is naturally emitted by the human body favors the use of LWIR for face recognition. LWIR systems can function even in complete darkness. In this thesis, all four of the implemented works were tested with LWIR images. 


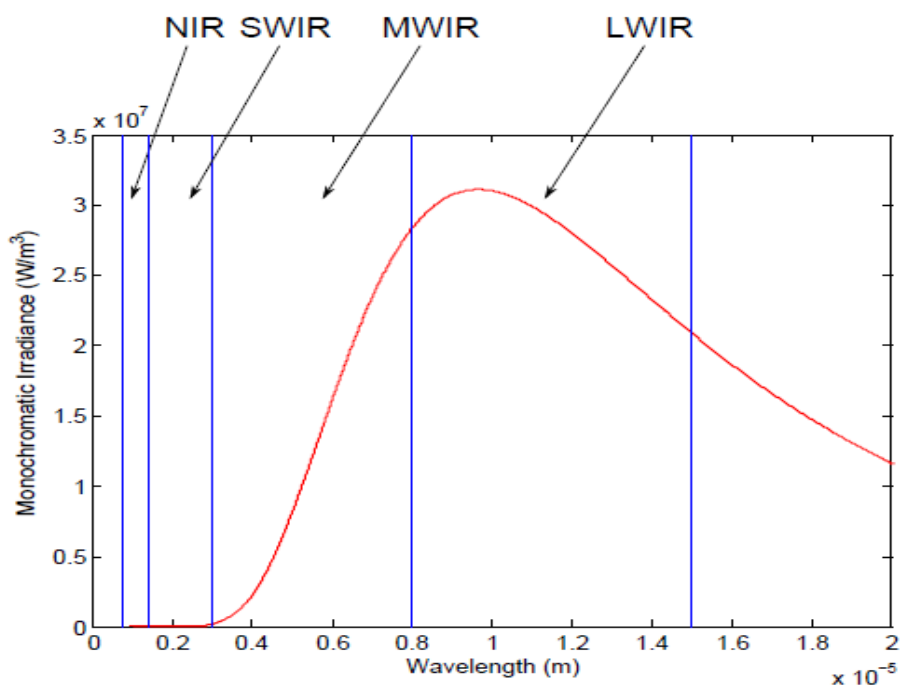

Figure 2.1 Human body Planck distribution [10]

\subsection{Advantages and Limitations of Infrared Face Recognition}

In this section advantages and limitations of infrared face recognition compared to visible spectrum face recognition are investigated.

\subsubsection{Advantages of Infrared Face Recognition}

Infrared images have some advantages over its visible counterpart for the purpose of face recognition. Some of the significant advantages are listed below.

- Infrared face recognition is robust under luminance variance. Figure 2.2 illustrates that the histogram of the infrared images changes insignificantly, unlike visible images, under luminance variance. Notice that under different illumination conditions the histograms are nearly identical. 


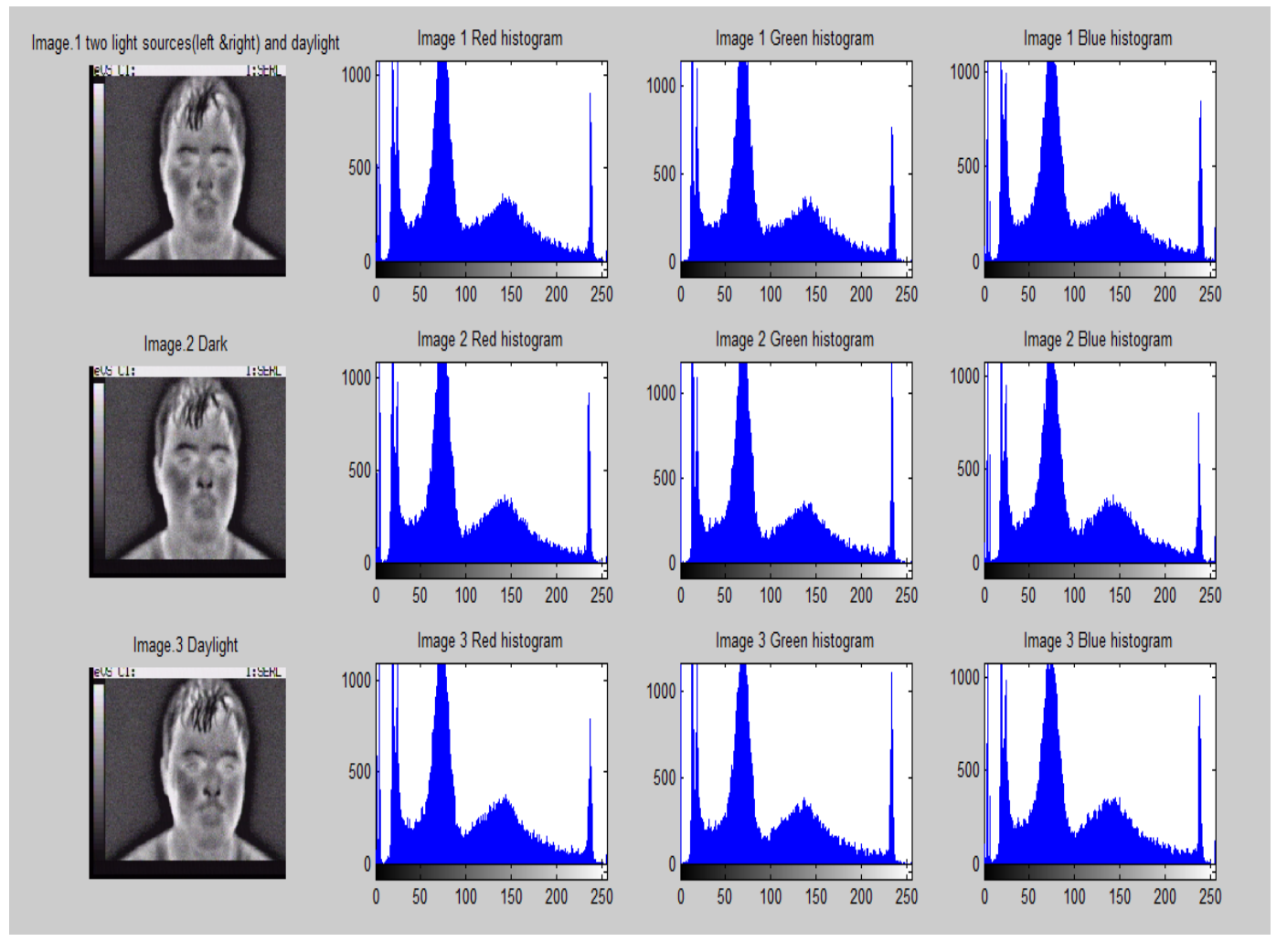

Figure 2.2 Histogram of the LWIR images under luminance variance

- Infrared images are more robust to pose and expression changes for the purpose of face recognition than its visible counterpart [15].

- Infrared face images can be utilized to obtain information about underlying vessel structure. This information is unique and it is immune to aging since the growth can be predicted [16]. The method is called vascular network and will be investigated thoroughly in this thesis work.

- Infrared face recognition systems are better at detecting disguises [17]. This is a result of the fact that, human face has lower reflectivity than the facial disguise materials. Also the human hair and artificial hair has different reflectivity characteristics in NIR band. 


\subsubsection{Limitations of Infrared Face Recognition}

There are also conditions where the visible face recognition systems perform better than the infrared face recognition systems.

- Skin temperature can change according to the ambient temperature. Thermal face images are affected by ambient temperature and that leads to a degradation of performance. This is stated in many works such as Prokoski \& Riedel [18], Socolinski \& Selinger [19], and Wu et al. [20]. The variance in the thermal image caused by ambient temperature variation is a problem for the same person's images taken under different ambient temperatures. To see the aforementioned phenomenon refer to images given in the work of $\mathrm{Wu}$ et al. [21] in Figure 2.3. It should also be noted here that there are algorithms that are developed to prevent this performance degradation such as blood perfusion.

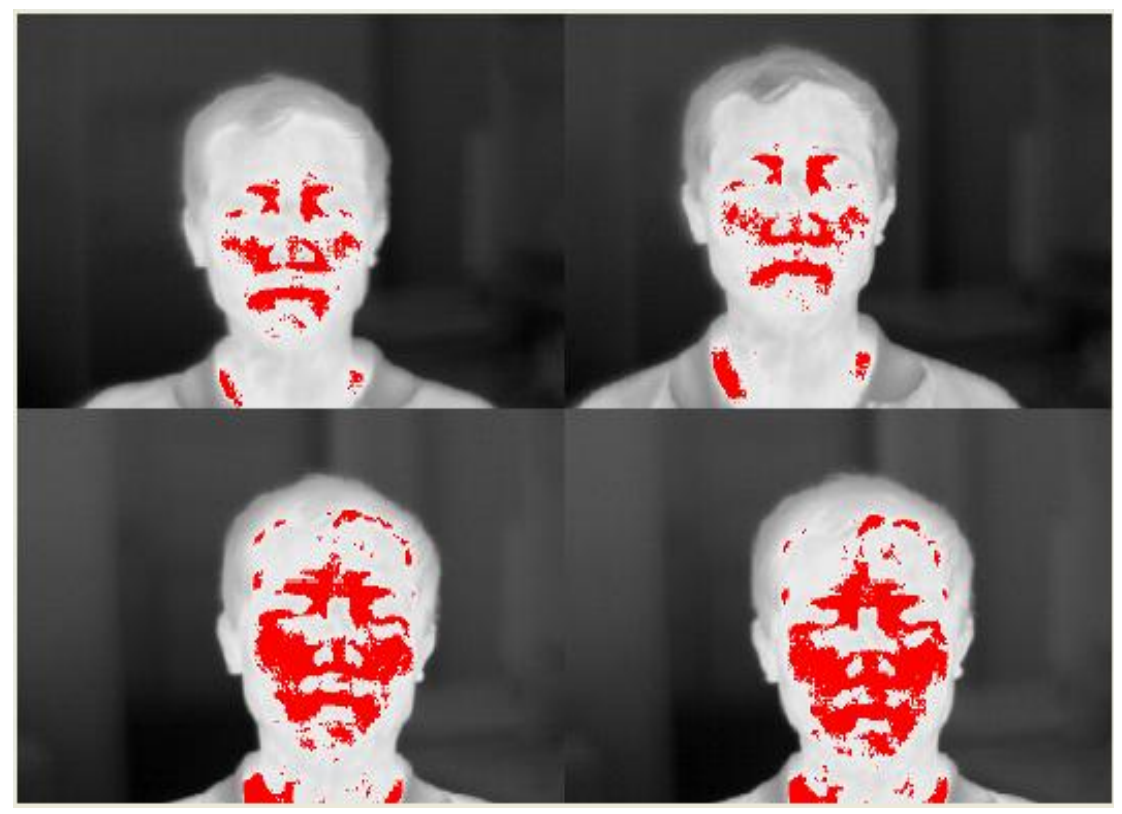

Figure 2.3 Images taken under different ambient temperatures [21]. Ambient temperatures are, from left to right, first row 28.4 and $28.7^{\circ} \mathrm{C}$; second row 28.9 and $29.3^{\circ} \mathrm{C}$. 
- In thermal images eyeglasses are opaque. This leads to some information loss in the areas of the face covered by the eyeglasses.

- Illumination variance also poses a problem for SWIR and NIR images as they are also affected by the sunlight like visible spectrum images [22]. In this thesis work, LWIR images are utilized. In LWIR images this problem does not occur.

- Thermal images are affected by metabolism, physical activity and emotional status [23]. Physical activity increases the human body temperature. Also excitement is known to increase the body temperature slightly. The utilized databases in this thesis work do not contain images that test this phenomenon.

- Thermal images are also affected by alcohol consumption since it affects thermal distribution [21]. This phenomenon can be seen in images taken at different times after alcohol consumption. Such work has been done in work of Wu et al. [21]. The highlighted sections of facial images have pixel values between 248 and 255. The photographs are taken at different times after alcohol consumption, to be specific after 0 (top left), 15 (top right), 35 (bottom left) and 100 minutes (bottom right) after alcohol intake. In the resulting images the highlighted area grew as the time passes and effect of alcohol kicks in. This is illustrated in Figure 2.4. The databases utilized in this thesis work do not contain images that test this condition. 


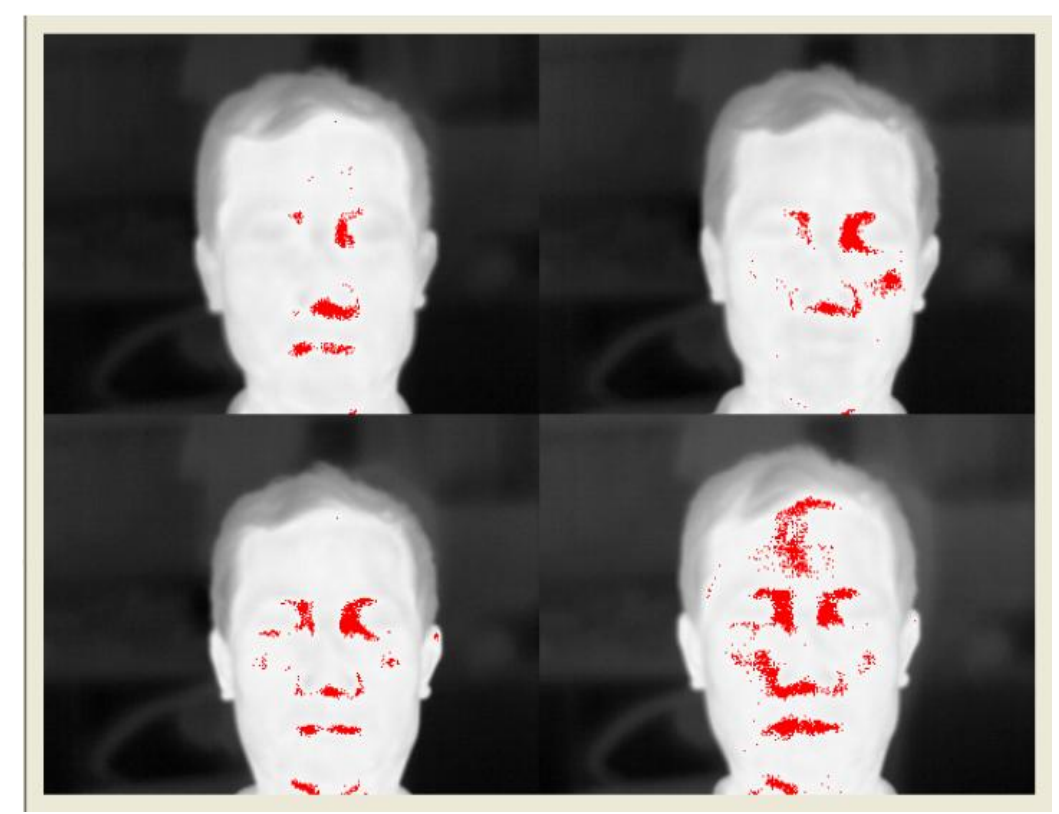

Figure 2.4 Photographs taken at different time intervals after alcohol intake [21]

\subsection{Infrared Face Recognition Techniques}

In this thesis work, infrared face recognition techniques are investigated in three groups; namely holistic approaches, feature based approaches and multimodal and intermodal approaches.

\subsubsection{Holistic Approaches}

In holistic approaches, the whole facial region is the input of the recognition system. Global representations are utilized to identify faces. Global representations are based on the entire facial region rather than local facial features. In that sense, holistic approaches regard the face as a whole. Every pixel belonging to face will contribute to the representation of the face, therefore all of such pixels are valuable.

The earliest works on infrared face recognition were holistic ones. The algorithms implemented in infrared spectrum closely followed the algorithms that were 
developed in visible spectrum. Most of the early works in holistic approaches are just the implementation of visible spectrum holistic algorithms with infrared images.

One of the most widely utilized holistic approaches in face recognition is Principal Component Analysis (PCA). PCA works as follows. An average face is calculated from the images that are presented in the training set. Difference from the average face is calculated for each image and covariance matrix is obtained. The eigenvectors are calculated from the covariance matrix. Those vectors represent the face space. Any face presented to the system can be described as linear combinations of those eigenvectors. Cutler [24] published one of the first works in infrared face recognition that utilizes PCA or eigenfaces. PCA technique, proposed by Turk et al. [25], relies on dimensionality reduction. Cutler worked on MWIR images and reached high rank-1 recognition rates. It was $96 \%$ for frontal and 45 degree orientation images and $100 \%$ for profile views. Wilder et al. utilized PCA technique with LWIR images. Socolinsky et al. [26], [27], [28] and Selinger et al. [29] implemented PCA with LWIR and visible images and obtained significantly better performance with LWIR images. On weighted average, the recognition rate for visible images was $73 \%$, whereas the recognition rate for infrared images was $95 \%$. It is argued that the reason for the dramatic performance difference between two modalities is the fact that the utilized data is not as testing for infrared spectrum as it is for visible spectrum [7]. Socolinsky et al. [27] also tested the performance of thermal images with time lapse data. Although it is seen as one of the limitations of thermal images for the purpose of face recognition, it is concluded that the degradation of performance with time lapse data is not as severe as thought but rather statistically insignificant compared to visible images. PCA was also implemented with SWIR images by Kang et al. [30]. Promising results are presented in this paper that supports the use of infrared images for face recognition. PCA is a viable technique that can be utilized for infrared face recognition. However, the later works reported significantly lower recognition rates. A common critic for early works is that the datasets used in those works were not challenging. 
LDA, also referred as Fisherfaces, is another algorithm that has been implemented with infrared images. LDA, like PCA, is an algorithm that is utilized for dimensionality reduction. In PCA the information is represented in an orthogonal linear space. In LDA the discriminative information is represented in a linearly separable space. The within and between scatter matrices are computed. The projections of the faces are utilized with a measure to create discriminative power. LDA works better than PCA for recognition task, as the aim of the LDA is to project the same class data to a point and project different classes' data to other points as far apart as possible. Socolinsky et al. [26] [27] [28] and Selinger et al. [29] worked on LWIR images and implemented LDA. In their comparative work, LDA was the best performing algorithm in both visible and thermal modalities. Another important thing to note was the superior performance of thermal images over visible spectrum images for all of the methods. Kang et al. [30] illustrated that LDA is also applicable to SWIR images with good results. In NIR band Zou et al. [31] tested LDA with four different classification algorithms; namely radial basis function neural networks, Adaboost, nearest neighbor and Support Vector Machine. NIR images performed better than the visible images in all four methods.

ICA is a statistical approach that assumes non Gaussian and statistically independent subcomponents whose linear combinations represent the data variables. ICA is also capable of finding hidden factors. ICA is a generalization of the PCA approach. PCA is a representation that only depends on pair wise relationship between pixels. In ICA high order relationships among pixels are also considered. That is the reason why ICA mostly outperforms PCA methods. Socolinsky et al. [26] [27] [28] and Selinger et al. [29] implemented ICA with LWIR and visible images. In their comparative work thermal images performed better than visible images, with respective recognition rates of $94 \%$ and $88 \%$. For visible images ICA performed much better than PCA.

Discrete Cosine Transform is also employed for face recognition in infrared images by Zhoa et al. [32]. Discrete Cosine Transform coefficients are utilized as the global 
features of the face. Lowest DCT frequencies are utilized for representation and support vector machine is utilized for classification. It is illustrated in this work that the method is applicable to NIR images with good experimental results.

Recent techniques applied to infrared images are more sophisticated statistical techniques that are developed for visible spectrum images. One of those techniques is implemented by Elguebaly and Bouguila [33]. Bayesian method is used to determine the parameters from training data sets of a Gaussian mixture model. Rank 1 recognition rate of $95 \%$ is achieved. Lin et al. [34] investigated the utilization of compressive sensing with infrared face images. Compressive sensing is a signal processing technique that is based on the idea of exploiting the scarcity of the signal, so that it can be obtained with fewer samples. Reported results were better compared to wavelet based decomposition approaches.

The use of multispectral images with holistic methods is also investigated by Pan et al. [35]. 31 narrow sub bands of NIR band are utilized and eigenfaces are calculated in those sub bands. The method showed discriminative power. "Spectral face", which is the sequential interlacing of the sub band images, is also proposed. When used as input to PCA "spectral faces" performed better than thermal faces.

\subsubsection{Feature Based Approaches}

Feature based approaches, first process images to extract local features. Those local features contain the distinctive information that is utilized for face recognition. The local features are extracted from the eyes, nose, mouth and other fiducial marks. The positions and the geometry of those features and the geometric relations between different features are fed to a structural classifier. This way the input to the recognition system is reduced to a vector of geometric features rather than the whole face. This means that not every pixel belonging to facial region is utilized for 
recognition task. In that sense, feature based approaches are different than holistic approaches.

One of the early works in infrared face recognition literature that utilized a feature based approach is proposed by Yoshitomi et al. [36]. Proposed method relies on detecting the temperature distribution of the face. Locally averaged temperature, recorded temperature distribution and shape factors are utilized for classification. Temperature distribution and locally averaged temperature are fed to neural network classifiers as separate inputs. Shape factors are utilized for supervised classification. The data from neural networks and supervised classification is combined to identify the face. High recognition rates as much as $92 \%$ are achieved. It is stated in this work that luminance related limitations of visible spectrum images were nonexistent in thermal images. Infrared images are also tested under different temperatures varying from $302 \mathrm{~K}$ to $285 \mathrm{~K}$. The performance of the proposed system greatly suffered and dropped to $60 \%$ percent.

Friedrich et al. [37] utilized template matching with LWIR images. The method is based on finding small features of the image that matches with the template image. Euclidian distance is employed for classification. It is concluded that the infrared images perform better than visible spectrum images under pose and expression variance.

Heo et al. [38] also made a comparative work on LWIR and visible images. Commercially available FaceIT software and Equinox database's co registered infrared and visible images are utilized in this work. The FaceIT software relies on local features extracted from face. It is observed that in the absence of eyeglasses thermal images outperformed the visible spectrum images.

Another feature based method is local binary patterns. Li et al. [22], [39], [40], [41] worked on local binary patterns with NIR images. Local binary patterns are texture descriptors. These texture descriptions are utilized as local features. In this method 
first the neighborhood of a pixel is investigated and the neighboring pixels are represented with a binary number according to their value compared to the center pixel. The histogram of these local representations is calculated. In this way a feature vector is obtained. Active NIR illumination is utilized, in order to locate the eye positions easily with flashing pupils caused by illumination. This also removed the need to compensate for illumination variance since the illumination was designed to be as homogenous as possible. Although high recognition rates are achieved, it is stated that the system required cooperative users and also active illumination limits the outdoor usage. Goswami et al. [42] made a comparative work on linear binary patterns with many linear and kernel methods.

Wavelet transform is a popular technique in visible spectrum face images. They contain information on both spatial and frequency aspects. Sirivastava et al. [43], [44] investigated the utilization of Gabor filter based wavelet transform to extract facial features with LWIR images. Bessel $\mathrm{K}$ forms were utilized for modeling the marginal density functions of those features. $\mathrm{L}_{2}$ norm is employed for matching phase. High recognition rates are reported and the described algorithm outperformed PCA and ICA. This illustrated that the method is applicable to infrared images as well. Nicolo and Schmid [45] also worked on Gabor wavelets. Weber local descriptors and linear binary patterns are employed for encoding the responses. Curvelet transform, which is based on wavelet transform, is also implemented with IR images by Xie et al. [46]. The scale of the curvelet determines the degree of oriental localization. Nearest neighbor classifier is utilized. Significant computation and storage improvement is achieved with the utilization of this method.

Vascular network is another popular technique in infrared face recognition. This approach is first proposed by Buddharaju et al. [47] and relies on physiological information of a person. The approach utilizes morphological operations to obtain information about underlying vein structure. The obtained vascular network is a contour shape that is unique for each person. The branching points of the vascular network, called Thermal Minutia points, are extracted from the image. Then those 
minutia points are compared to the training image gallery in order to match the person. Vascular network technique is one of the investigated techniques in this thesis work. Vascular network approach and significant works on vascular networks will be investigated in following chapters. Here vascular network approach is only stated for the sake of completion.

Blood perfusion, like vascular networks, is another algorithm that is specifically developed for infrared images. Blood perfusion, proposed by Wu et al. [20], is a technique utilized to overcome limitations of infrared face recognition. It is a point wise transform that is designed to eliminate ambient effects, which degrades the performance of the infrared face recognition systems. Better results are achieved with the utilization of blood perfusion images compared to the utilization of thermal images. Blood perfusion, like vascular network technique, is investigated in this thesis work in detail in following chapters. It is just mentioned here for the sake of completion.

\subsubsection{Multimodal and Intermodal Techniques}

Multimodal techniques utilize both visible and infrared spectrum images and some sort of a fusion scheme. The aim is to overcome the limitations experienced by those modalities individually and merge their strong suits. Fusion scheme can be data driven, which means the pixel values are fused in some combination; or decision based, which means the results of individual modalities are fused according to obtained scores from each modality.

Intermodal techniques on the other hand, deal with the matching of images that belong to different modalities. In those techniques it is investigated whether the data in one modality is sufficient to decide the identity of the person in another modality. 


\subsubsection{Multimodal Techniques}

Multimodal techniques are fusion based techniques. Multimodal techniques can be investigated in two groups, namely decision based fusion and data based fusion.

\subsection{Decision Based Fusion}

Chen et al. [48], [49] proposed a rank based fusion and a score based fusion. Rank based fusion is based on deciding with the sum of ranks obtained from individual modalities and score based fusion is based on the sum of the scores obtained in individual modalities. The match score here is obtained through a distance algorithm. Euclidian and Mahalanobis distances are utilized. PCA is utilized for representation. Higher recognition rates are achieved with fusion compared to utilization of individual modalities. Score based fusion was the best performing algorithm in this work. Socolinsky et al. [19], [27] also proposed a very similar score based fusion that utilized PCA and Mahalanobis angle distance. Fusion scheme led to better results compared to individual modalities. In all of these works LWIR and visible images were utilized.

Chen et al. [50] proposed the use of fuzzy integral fusion. LWIR and visible images are utilized. Eigenface method was implemented to each space. The components of eigenfaces constitute a feature vector. A modified histogram based technique is applied to obtained vectors. Increased recognition rates are reported in this work.

Buddharaju et al. [51] proposed a fusion method which employs PCA for visible images and vascular networks for thermal images. The scores obtained in each modality are combined to obtain superior performance compared to the use of an individual modality.

There are algorithms in the literature that utilizes more than 2 modalities. Infrared, visible and 3D imagery is fused in some of the works such as Bowyer et al. [52], 
Kakariadis et al. [53], [54]. In all of these works, the fusion of all three modalities is the best performing method.

\subsection{Data Based Fusion}

Data based fusion is another fusion scheme that is employed in literature. Early works were proposed by Gyaourova et al. [55] and Singh et al. [56] to overcome the occlusions that the eyeglasses cause in infrared images. They used Haar wavelets coefficients for LWIR and visible images. Genetic algorithms method was utilized in order to fuse those coefficients. Better results are reported in presence of eyeglasses.

Singh et al. [57] proposed a hierarchical approach. With the utilization of 2D $\log$ Gabor wavelets, amplitude and phase features are obtained. Those features were fused with an adaptive support vector machine. The same procedure is applied to different wavelengths. Another step of support vector machine is utilized as higher hierarchy fusion. In this work, the fusion of SWIR and visible images performed the best.

Bebis et al. [58] proposed a data fusion technique based on PCA. Eigenfaces were obtained for visible and thermal infrared images. The appropriate eigenfeatures were selected by genetic algorithms method. Proposed fusion method performed better than PCA applied to individual modalities.

Hariharan et al. [59] proposed a method based on empirical mode decomposition. With the utilization of empirical mode decomposition intrinsic mode functions are obtained for LWIR and visible images. Those functions are fused according to weights that are decided empirically. For the matching part FaceIt software is utilized. The described fusion scheme performed better than PCA fusion, averaging and wavelet fusion. 
There are also fusion methods that utilize both decision based and data based fusion methods. Methods proposed by Heo et al. [60] and Arandjelovic et al. [61] are examples of such work that employ both fusion schemes. Heo et al. [60] employed data fusion step first. The fused data, visible and thermal data are classified with FaceIt software. In the last step, a decision fusion step is employed which merges individual results. Arandjelovic et al. [61] utilized a similar fusion scheme with feature based approaches.

\subsubsection{Intermodal Techniques}

Intermodal techniques aim to match images that belong to infrared spectrum with their corresponding visible spectrum images. Bourlai et al. [62] investigated this phenomenon with SWIR and visible images. For detection Viola Jones method is utilized and in the classification step $\mathrm{K}$ nearest neighbor, Verilook's commercial system and Identity tool's G8 commercial system are utilized. The results illustrated that interspectral recognition between SWIR and visible images is feasible.

NIR and visible image interspectral recognition is investigated by Klare and Jain [63]. Histogram of oriented gradients and local binary patterns are utilized in the work. This method also led to promising results.

Bourlai et al. [64] investigated interspectral recognition between MWIR and visible images. A three step algorithm is utilized with all three of the steps having several alternatives. Preprocessing step alternatives are self quotient image and difference of Gaussian filtering; feature extraction step alternatives are local binary patterns, oriented gradients, scale invariant feature transform; classification step alternatives are Euclidian and city block distance, chi-squared. None of the combinations of the given alternatives gave an acceptable result with the highest recognition rate $40 \%$. It is argued that the more the difference between spectrums is, the harder the task of 
interspectral recognition is [7]. This makes sense as the difference between images tends to increase when the difference between two modalities are higher. 


\section{CHAPTER 3}

\section{BACKGROUND}

In this chapter the utilized face segmentation method and background on implemented methods is discussed. First the segmentation method is explained. In the second part of this chapter general descriptions of the implemented methods and significant works in literature regarding each method are presented.

\subsection{Face Segmentation Algorithm Employed in Implemented Recognition Methods}

Face segmentation is a step common to all of the face recognition algorithms. Face segmentation, as the name suggests, aims to differentiate the facial regions from other regions. The performance of this step is important since segmentation determines the regions of interest. Face recognition utilizes facial data to discriminate persons and the determination of the regions that belong to face, in other words the data to be utilized for recognition task, is critical. A poorly performing face segmentation algorithm degrades the performance of the overall face recognition algorithm.

In this section, the utilized segmentation method will be described. The method is based on the approach by Filipe [65]. The method starts with the application of "Rectangular region of interest" (RROI) operator. The operator is designed to eliminate neck and cloths in the resulting segmented image. The operator first defines two vectors called horizontal and vertical signature vectors. For an NxM 
image there are $\mathrm{N}$ vertical and $\mathrm{M}$ horizontal values to be calculated that constitute the respective vectors. The horizontal and vertical signature vectors can be expressed mathematically as in Equation (3.1).

$$
\begin{aligned}
& H(n)=\sum_{n=1}^{N} I(n, m), \\
& V(n)=\sum_{m=1}^{M} I(n, m) .
\end{aligned}
$$

After obtaining the vertical signature vector a Gaussian filter is utilized to smoothen the vector. This filtering removes high frequency components in the vertical vector. Desired information is the extrema points of the first derivative of the smoothened vector. First derivative is taken and extrema points are decided. This procedure decides the left and right side limits of the face.

A similar procedure is followed to obtain the line that corresponds to top limit of the head. For this purpose, horizontal signature vector and a filtering operation is employed. First derivative is taken to find the extrema point. Resulting top, left and right limits are given in Figure 3.1.

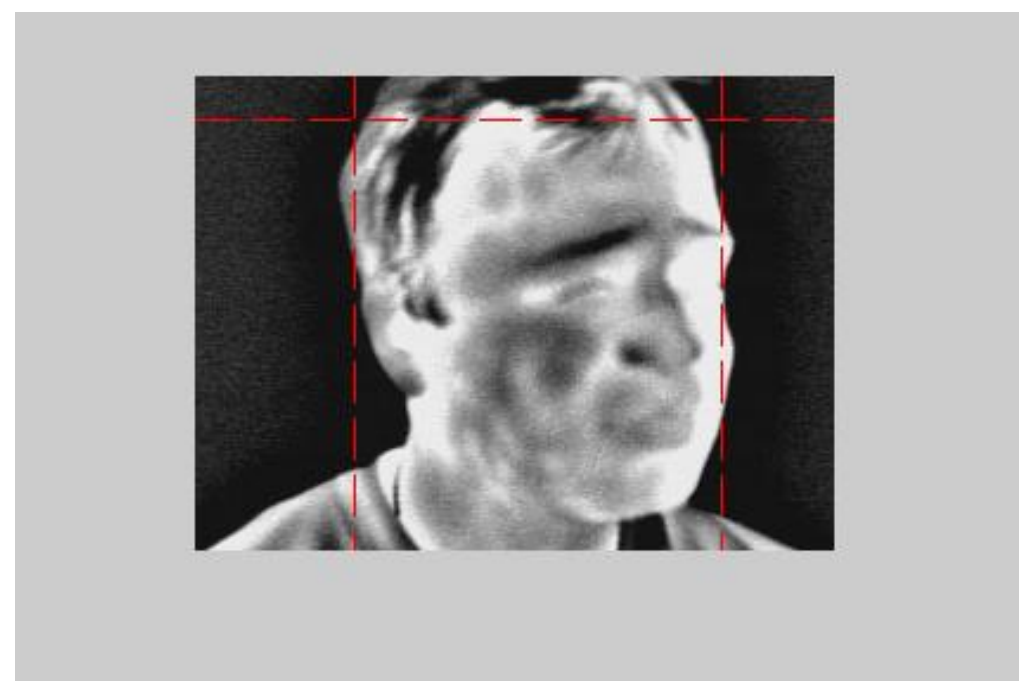

Figure 3.1 Obtained facial limits 
In order to find the lower limit of the face, a curve fitting algorithm is employed. Given test image is cropped, so that the remaining part of the image is the bottom one third of the rows. The bottom limit is assumed to be in this region. Before obtaining the edges with canny edge detection algorithm; linear reduction in the number of colors and Gaussian blur are employed as preprocessing steps. The edges obtained from the canny edge detection algorithm are utilized in Least squares estimation curve fitting method. A second order function in the form $f(x)=a x^{2}+$ $b x+c$ is assumed to describe the set of edges where $\mathrm{x}$ and $\mathrm{y}$ are spatial coordinates. Least square error can be found as in Equation (3.2).

$$
S=\sum_{i=1}^{M}\left(y_{i}-\left(a x_{i}^{2}+b x_{i}+c\right)\right)^{2}
$$

The smaller the error is, the better the curve fits. The gradients with respect to parameters $a, \mathrm{~b}$ and $\mathrm{c}$ are equalized to zero to find the minimum cumulative error. The gradients and the obtained equations with gradient term equalized to zero are given in Equation (3.5), Equation (3.4) and Equation (3.5).

$$
\begin{gathered}
\frac{\partial S}{\partial a}=2 \sum_{i=1}^{M}\left(y_{i}-\left(a x_{i}^{2}+b x_{i}+c\right)\right)\left(-x_{i}^{2}\right) \\
a \sum_{i=1}^{M}\left(x_{i}^{4}\right)+b \sum_{i=1}^{M}\left(x_{i}^{3}\right)+c \sum_{i=1}^{M}\left(x_{i}^{2}\right)=\sum_{i=1}^{M}\left(y_{i} x_{i}^{2}\right) . \\
\frac{\partial S}{\partial b}=2 \sum_{i=1}^{M}\left(y_{i}-\left(a x_{i}^{2}+b x_{i}+c\right)\right)\left(-x_{i}\right) \\
a \sum_{i=1}^{M}\left(x_{i}{ }^{3}\right)+b \sum_{i=1}^{M}\left(x_{i}{ }^{2}\right)+c \sum_{i=1}^{M}\left(x_{i}\right)=\sum_{i=1}^{M}\left(y_{i} x_{i}\right) .
\end{gathered}
$$




$$
\begin{aligned}
& \frac{\partial S}{\partial c}=2 \sum_{i=1}^{M}\left(y_{i}-\left(a x_{i}{ }^{2}+b x_{i}+c\right)\right)(1) \\
& a \sum_{i=1}^{M}\left(x_{i}{ }^{2}\right)+b \sum_{i=1}^{M}\left(x_{i}\right)+c M=\sum_{i=1}^{M}\left(y_{i}\right) .
\end{aligned}
$$

From these three equations $a, b$ and $\mathrm{c}$ parameters can be calculated. With the obtained parameters, the lower limit of the face could be found. Parameter ' $a$ ' contains information about the curvature of the limit. If $a<0$ then the curve is fitted to chin, if $a>0$ the curve is fitted to shoulders. Figure 3.2 illustrates the curve fitting procedure.

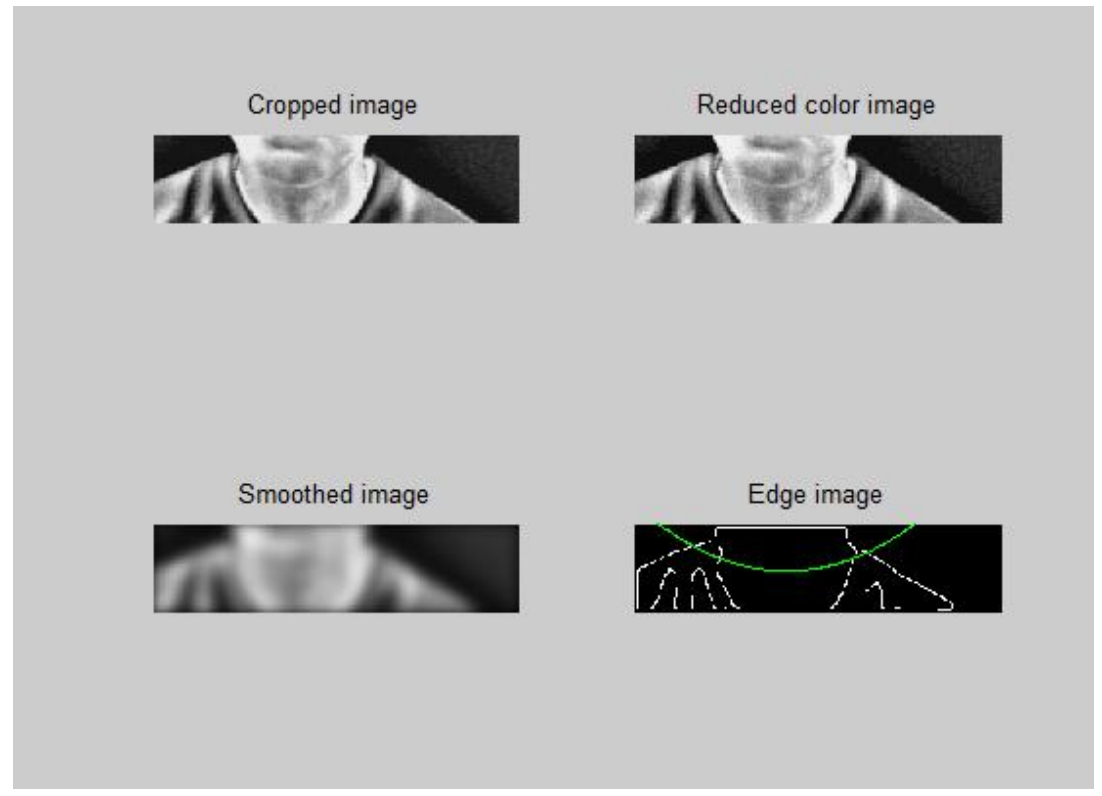

Figure 3.2 Curve fitting procedure applied to the cropped image

The next step in the algorithm is to utilize Chan-Vese [66] active contour method in order to segment the face.

The method aims to minimize the energy function. A closed shape in an image $u_{0}$ is considered and the region inside the image is represented with C. $c_{1}$ is the mean value of the region inside the closed shape and $c_{2}$ is the mean of the region outside 
of the closed region. There are two functions with $F_{1}(C)$ representing the force to shrink the shape and $F_{2}(C)$ representing the force that expands the shape. The mathematical representation can be seen in Equation (3.6).

$$
\begin{aligned}
F_{1}(C)+F_{2}(C) & =\int_{\text {inside }(C)}\left|u_{0}(x, y)-c_{1}\right|^{2} d x d y \\
& +\int_{\text {outside }(C)}\left|u_{0}(x, y)-c_{2}\right|^{2} d x d y
\end{aligned}
$$

When two forces become zero the closed shape is considered fitted.

In the active contour method the energy function is stated as in Equation (3.7).

$$
\begin{array}{r}
F\left(c_{1}, c_{2}, C\right)=\mu . \text { Length }(C)+v \cdot \text { Area }(\text { inside }(C)) \\
+\lambda_{1} \int_{\text {inside }(C)}\left|u_{0}(x, y)-c_{1}\right|^{2} d x d y \\
+\lambda_{2} \int_{\text {outside }(C)}\left|u_{0}(x, y)-c_{2}\right|^{2} d x d y .
\end{array}
$$

The derivative of above expression is taken in order to obtain the minimum point. $\mu$ is taken 0.2. The result after 200 iterations can be seen in Figure 3.3.

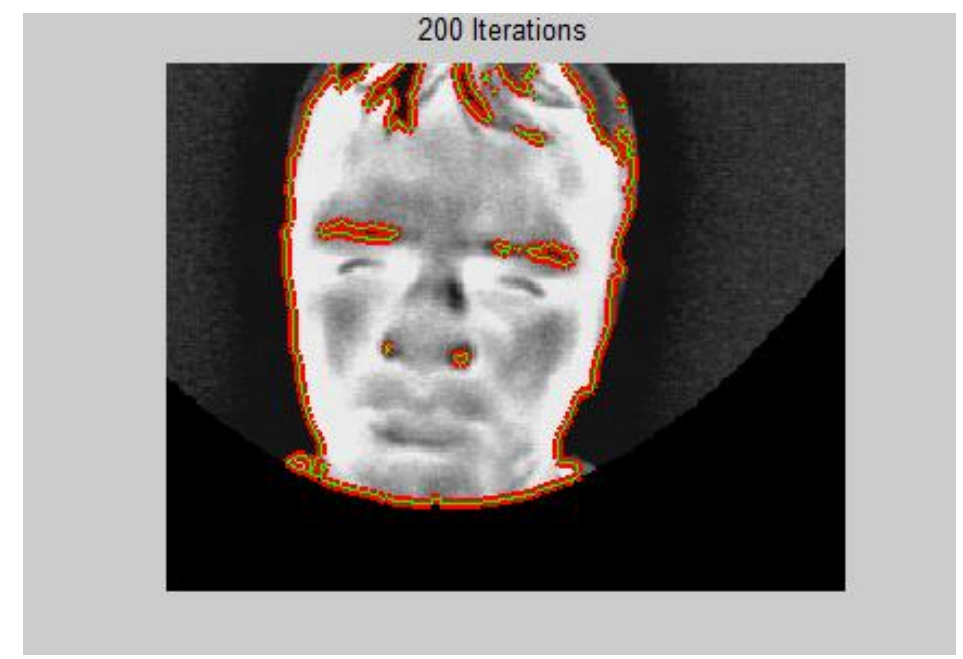

Figure 3.3 Segmented face with the explained method 
Post processing procedure follows the active contour method. The center of the face is found from the limits obtained before. Canny edge detection and dilation operations are employed to find and enhance edges. The largest edge that contains the center point is considered the facial region. This region is compared with the active contour method's result and the small segments are removed. Eye glasses are opaque in infrared spectrum so the pixels in those regions are occluded.

The implementation of the algorithm explained above is taken from the thesis work of Melis Eryılmaz [67]. In the work done by Eryılmaz [67] this algorithm is compared with several algorithms and it is the best performing algorithm. The performance of the algorithm is significantly better than the compared algorithms. Two error rates are defined in her work with $E_{1}$ as the segmentation error rate and $E_{2}$ as the error rate defined by the mean of false positive rate and false negative rate. One of the algorithms, that the above explained method is compared with, is the segmentation algorithm that is utilized by Cho et al. [75]. The significance of this work is that it is also one of the recognition schemes utilized in this thesis work. It can be observed that the segmentation algorithm utilized in this thesis work performs significantly better compared with Cho et al.'s [75] work with nearly one third of the respective error rates. The algorithms are compared in IRIS and Terravic databases. In this thesis work those databases are the ones that are utilized as well. For the employed segmentation algorithm, IRIS database error rates are $E_{1} 0.111$ and $E_{2} 0.139$, and for the Terravic database error rates are $E_{1} 0.066, E_{2}$ is 0.068 . The differences in the error rates for these two databases do not cause a significant problem. The reason is that the above mentioned difference is not significant when the segmented face is utilized in a recognition scheme, but it is merely important for the segmentation task. For the recognition scheme it is not critical whether a segmentation algorithm follow the hairline exactly. Whereas, for the segmentation task every pixel classified as face or non face matters, as the performance is determined by the number of pixels classified right and wrong. Every point is important for a segmentation scheme. Those points may not contain meaningful 
information for recognition schemes, though. The discrepancy in the results is not structural pixels. Also the images to be utilized for recognition task are chosen, so that the images in which the segmentation scheme performs very poorly are eliminated. The above segmentation results are given for images from Terravic database. In Figure 3.4 original image from IRIS database and the mask obtained from that image are illustrated.

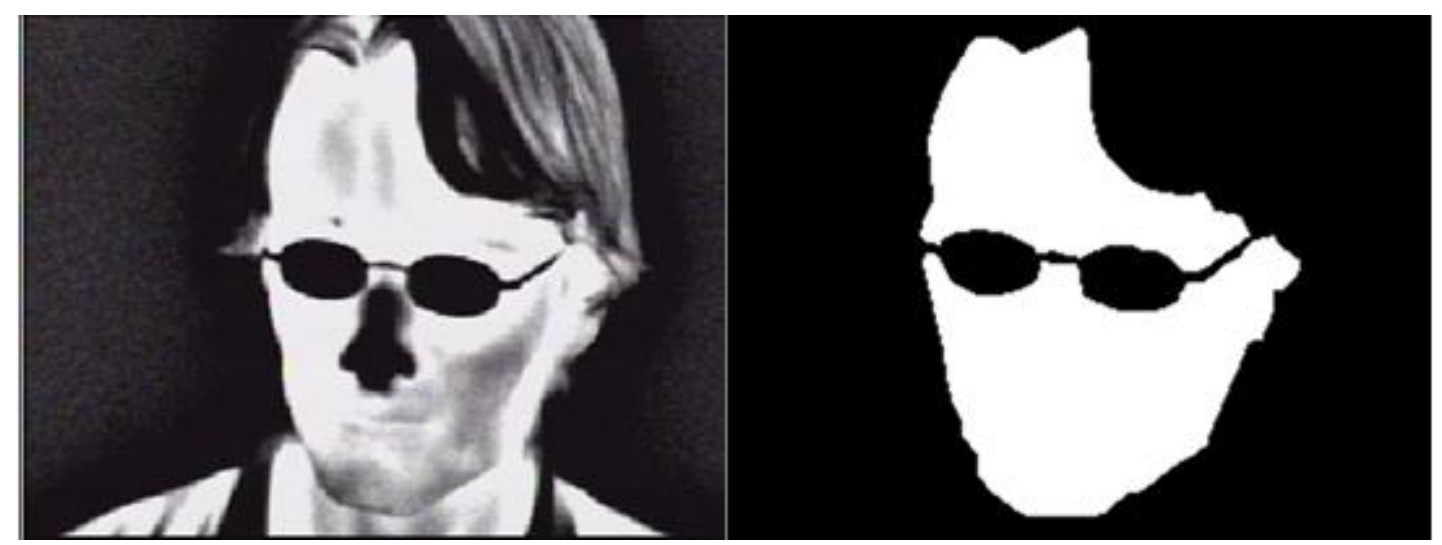

Figure 3.4 Original image from IRIS database and obtained mask with the utilization of the segmentation method

\subsection{Background Information on Implemented Methods}

Three state of the art methods are investigated in this thesis work. General description of these methods and significant works in literature are explained in this section.

\subsubsection{PCA Method}

The first method that is implemented in this thesis work is Principal Component Analysis (PCA), eigenfaces. PCA is a popular statistical procedure that has a broad area of utilization. One of them is face recognition in visible spectrum. The idea was

first introduced by Turk and Pentland [25]. This was one of the first works regarding 
face recognition of visible images. As mentioned earlier, many face recognition algorithms that is implemented in the visible spectrum, was adapted to infrared spectrum images and PCA is no exception. PCA is implemented in infrared spectrum and it was one of the earliest works presented in the literature. One of the first works that relied on PCA for the purpose of face recognition in infrared spectrum was made by Cutler [25]. Cutler introduced the technique with MWIR images. Later Socolinsky et al. [26], [27], [28] and Selinger et al. [29] implemented PCA with LWIR images. These works illustrated that eigenface method is suitable to be utilized with thermal images for the purpose face recognition, as high recognition rates are achieved. The details and more work were investigated in chapter 2 of this thesis work.

The general description of the PCA method can be made as follows: First, the covariance matrix and its eigenvectors are obtained from the given training images. Eigenvectors encode the variances between the faces and they form a basis. Any image that is presented can be represented by linear combinations of the basis set. Classification is made by comparing this representation.

A simple flowchart for implementing PCA for face recognition is given in Figure 3.5 .

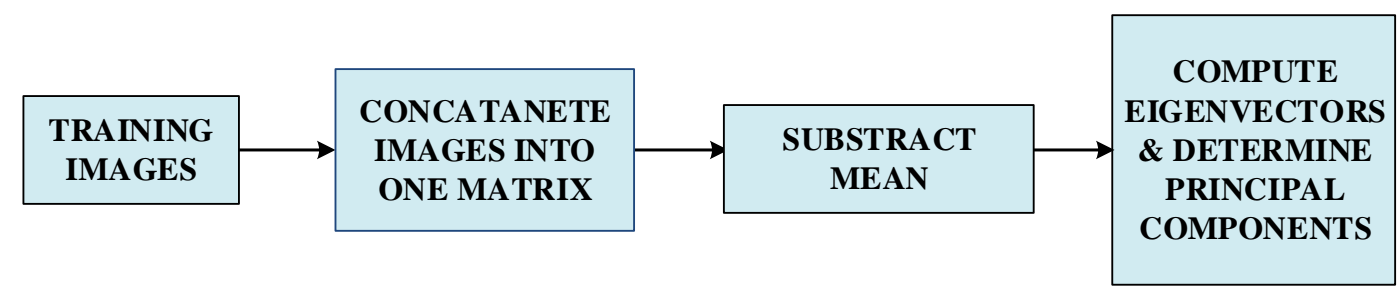

Figure 3.5 A simple flowchart for implementing PCA for face recognition

Images can be thought of vectors. An $\mathrm{N}$ by $\mathrm{N}$ image is a vector of dimension $\mathrm{N}^{2}$. This corresponds to a point in $\mathrm{N}^{2}$ space. So a set of images can be thought as points 
in $\mathrm{N}^{2}$ space. Those points in the space are not randomly distributed since the faces have a structural resemblance. That leads to the idea that there is a significant statistical redundancy in representation of the images and they can be represented by a lower dimensional subspace. The aim of utilizing principal component analysis is to obtain the basis vectors of this aforementioned subspace. These basis vectors are the eigenvectors of the covariance matrix that encodes the variances between the faces.

Consider $\mathrm{K}$ training images that are represented as $\mathrm{I}_{1}, \mathrm{I}_{2} \ldots \mathrm{I}_{\mathrm{K}}$. An average image can be expressed as in Equation (3.8).

$$
\varphi=\frac{1}{K} \sum_{J=1}^{K} \mathrm{I}_{\mathrm{j}} .
$$

Each image is different from the mean by a vector $\varphi$. This is presented in Equation (3.9).

$$
\delta_{i}=I_{i}-\varphi
$$

The covariance matrix can be written as in Equation (3.10).

$$
\mathrm{C}=\sum_{j=0}^{K} \delta_{\mathrm{j}} \delta_{\mathrm{j}}^{\mathrm{T}} .
$$

Note that it is a tedious work to calculate the eigenvectors of the above covariance matrix. For an $\mathrm{NxN}$ image the covariance matrix stated above is $\mathrm{N}^{2}$ by $\mathrm{N}^{2}$. Considering widely used image sizes calculating the eigenvectors of the covariance matrix in this form is not computationally feasible. There is a way to work around this, though. The eigenvectors of the matrix given in Equation (3.11) is computationally feasible and it can be shown that they are linearly related to the eigenvectors of the original covariance matrix. 


$$
\mathrm{D}=\sum_{j=0}^{K} \delta_{\mathrm{j}}^{\mathrm{T}} \delta_{\mathrm{j}} .
$$

For classification, a test image is projected to the face space as given in Equation (3.12). $\mathrm{T}$ corresponds to test image where $\mathrm{u}_{\mathrm{i}}$ 's are eigenvectors of the covariance matrix.

$$
w_{i}=u_{i}^{T}(T-\varphi)
$$

Weights are calculated in this manner to constitute a vector as in Equation (3.13). Note that $\mathrm{L}$ is the number of most significant eigenvectors that is sufficient to describe the set of training images.

$$
\rho=\left(w_{1}, w_{2}, \ldots, w_{L}\right)
$$

In order to decide the class of the given test image, simple Euclidian distance is employed as shown in Equation (3.14).

$$
\varepsilon_{i}=\left|\rho-\rho_{i}\right|
$$

A user defined threshold can be employed to decide whether the face belongs to that face class or not.

Robust PCA is a modified version of the widely used PCA algorithm. Robust PCA is proposed by Candes et al. [68]. The data matrix is assumed to be the superposition of a low rank matrix and a sparse matrix. The representation of the data matrix can be seen in Equation (3.15). The low rank matrix is represented with $\mathrm{L}$, sparse matrix is represented with $\mathrm{S}$ and the data matrix is represented with $\mathrm{M}$. 


$$
M=L+S \text {. }
$$

The aim of the Robust PCA method is to recover the individual components. This is done via solving an optimization problem, namely Principal Component Pursuit. This optimization problem is presented in Equation (3.16).

$$
\begin{gathered}
\text { minimize }\|L\|_{*}+\lambda\|S\|_{1}, \\
\text { where }\|L\|_{*}=\sum_{i} \sigma_{i}(L),\|S\|_{1}=\sum_{i}\left|S_{i j}\right|, \\
\text { subject to } M=L+S .
\end{gathered}
$$

Robust PCA algorithm is used in video surveillance and face recognition. The main idea of PCA in face recognition is to find a low dimensional space representation of the facial images. The robust PCA algorithm is able to determine this representation more accurately compared to PCA. The robust PCA algorithm can also be used with grossly corrupted data as it is able to retrieve the low rank matrix from the data matrix.

\subsubsection{Blood Perfusion Method}

Blood perfusion is a method that gathered a lot of attention in the infrared face recognition researches. It is the second investigated method in this thesis work. In this section general knowledge about the method and the important works related to this method are given.

Blood perfusion is developed for the infrared spectrum to reduce the effects of ambient temperature variations. The method also provides better results with time lapse data and reduces within class difference.

When a thermal image of a face is captured, what is obtained is the thermal radiance from the face skin. This also includes the effect of the ambient temperature. The 
ambient temperature is assumed to be colder than the temperature of the body. Blood perfusion is based on the static thermal physiology; constant, stable flux of heat, in other words thermal equilibrium. This idea is first presented in the work of Wu et al. [20]. Some assumptions exist in the blood perfusion model. Such as:

- Testing subjects are in steady state, in other words the deep body temperature is constant and no thermal regulation is considered such as sweating.

- The ambient temperature is lower than the body temperature.

- Pathological and psychological conditions are not considered. (The temperature of the face is observed to change under some pathological (like headache, fever, inflammation) and psychological (like blush, anger, stress) conditions.)

Under thermal equilibrium and with heat exchange only at the skin level, the Equation (3.17) can be written.

$$
\mathrm{H}_{\mathrm{r}}+\mathrm{H}_{\mathrm{e}}+\mathrm{H}_{\mathrm{f}}=\mathrm{H}_{\mathrm{c}}+\mathrm{H}_{\mathrm{m}}+\mathrm{H}_{\mathrm{b}}
$$

In the given equation $\mathrm{H}$ represents heat flux per unit area, subscripts $\mathrm{r}$, e and $\mathrm{f}$ refer to radiation, evaporation, convection respectively. The subscripts $\mathrm{c}, \mathrm{m}$, and $\mathrm{b}$ refer to conduction, metabolism and blood flow convection. Radiation, evaporation, convection are terms that represents the heat flow that outflows from the skin surface to the environment, whereas the terms conduction, metabolism and blood flow convection represent the influx heat flow, which is the heat flow from the body to skin surface. One can notice that those terms are assumed equal in other words thermal equilibrium is considered. 
From the heat equilibrium, extending the $\mathrm{H}$ terms $\mathrm{Wu}$ et al. [20] obtained a transform; which is the blood perfusion transform given in Equation (3.18).

$$
w=\frac{\varepsilon \sigma\left(\mathrm{T}_{\mathrm{s}}^{4}-\mathrm{T}_{\mathrm{e}}^{4}\right)+A \mu \mathrm{d}^{3 \mathrm{M}-1}\left(\mathrm{Pg} \beta / \mathrm{v}^{2}\right)^{\mathrm{M}}\left(\mathrm{T}_{\mathrm{s}}-\mathrm{T}_{\mathrm{e}}\right)^{\mathrm{M}+1}-\mathrm{k}\left(\mathrm{T}_{\mathrm{c}}-\mathrm{T}_{\mathrm{s}}\right) / \mathrm{D}-\mathrm{H}_{\mathrm{m}}}{\alpha c_{b}\left(T_{a}-T_{s}\right)} .
$$

The terms in Equation (3.18) and what they stand for are given in the Table 3-1.

Table 3-1 Terms in blood perfusion transform and their descriptions

\begin{tabular}{|c|c|c|}
\hline Symbol & Description & Value \\
\hline$w$ & Blood Perfusion & - \\
\hline$\sigma$ & Stefan-Boltzman Constant & $5.67 \times 10^{-8} \mathrm{Wm}^{-2} \mathrm{~K}^{-4}$ \\
\hline$\varepsilon$ & Tissue/ Skin Thermal Emissivity & 0.98 \\
\hline $\mathrm{T}_{\mathrm{s}}$ & Skin Temperature & - \\
\hline $\mathrm{T}_{\mathrm{e}}$ & Ambient Temperature & - \\
\hline $\mathrm{T}_{\mathrm{a}}$ & Artery Temperature & $312.15 \mathrm{~K}$ \\
\hline $\mathrm{T}_{\mathrm{c}}$ & Core Temperature & $312.15 \mathrm{~K}$ \\
\hline$k$ & Tissue/ Skin Conductivity & $0.2 \mathrm{~W} \mathrm{~m}^{-1} \mathrm{~K}^{-1}$ \\
\hline $\mathrm{M}$ & Air Thermal Conductivity & $0.024 \mathrm{~W} \mathrm{~m}^{-1} \mathrm{~K}^{-1}$ \\
\hline$c_{b}$ & Blood Specific Heat & $3.78 \times 10^{3} \mathrm{~J} \mathrm{~kg}^{-1} \mathrm{~K}^{-1}$ \\
\hline $\mathrm{H}_{\mathrm{m}}$ & Metabolic Heat Flux per Unit Area & $4.186 \mathrm{~W} \mathrm{~m}^{-2}$ \\
\hline$\alpha$ & Tissue / Skin Countercurrent Exchange Ratio & 0.8 \\
\hline $\mathrm{P}$ & Prandtl Constant & 0.72 \\
\hline$V$ & Kinematic Viscosity of Air & $1.6 \times 10^{-5} \mathrm{~m} / \mathrm{s}^{2}$ \\
\hline B & Air Thermal Expansion Coefficient & $3.345 \times 10^{-3} \mathrm{~K}^{-1}$ \\
\hline$g$ & Local Gravity Acceleration & $9.8 \mathrm{~m} / \mathrm{s}^{2}$ \\
\hline$A$ & Constant & 0.27 \\
\hline$M$ & Constant & 0.25 \\
\hline$d$ & Characteristic Length of Face & 0.095 \\
\hline$D$ & Distance from Body Core to Skin Surface & 0.095 \\
\hline
\end{tabular}


The obtained transform is point wise. This leads to two important advantages for thermal face recognition. The first one is the transform does not distort the location of the facial features. This means that if an algorithm which uses facial features is to be applied after applying blood perfusion, it can be employed without losing or infecting any data. The second advantage and may be the more important one is that the transform is nonlinear and emphasizes high temperature regions. The method achieves that with increasing the contrast for parts of the image that correspond to high temperature regions and suppress the contrast for parts of the image that correspond the low temperature regions. Those high temperature regions are the ones that contain the important information for thermal face recognition. In other words, the transform highlights between-class difference and decreases the withinclass difference. This can be shown with a simple differentiation with respect to temperature shown in Equation (3.19). The term $\mathrm{S}$ in Equation (3.19) is given in Equation (3.20).

$$
\frac{d w}{d T}=\frac{\left(4 \varepsilon \sigma \mathrm{T}^{3}+A \mu \mathrm{d}^{3 \mathrm{M}-1}\left(\mathrm{Pg} \frac{\beta}{\mathrm{v}^{2}}\right)^{\mathrm{M}}\left(\mathrm{T}-\mathrm{T}_{\mathrm{e}}\right)^{\mathrm{M}}+\frac{\mathrm{kT}}{\mathrm{D}}\right)\left(\mathrm{T}_{\mathrm{c}}-\mathrm{T}\right)+\mathrm{S}}{\alpha c_{b}\left(T_{a}-T\right)^{2}}
$$

$$
S=\varepsilon \sigma\left(\mathrm{T}^{4}-\mathrm{T}_{\mathrm{e}}^{4}\right)+A \mu \mathrm{d}^{3 \mathrm{M}-1}\left(\mathrm{Pg} \beta / \mathrm{v}^{2}\right)^{\mathrm{M}}\left(\mathrm{T}-\mathrm{T}_{\mathrm{e}}\right)^{\mathrm{M}+1}-\frac{\mathrm{k}\left(\mathrm{T}_{\mathrm{c}}-\mathrm{T}\right)}{\mathrm{D}}-\mathrm{H}_{\mathrm{m}}
$$

Considering the aforementioned assumption, the ambient temperature is lower than skin temperature $\mathrm{T}_{\mathrm{a}}>\mathrm{T}, \mathrm{S}$ term is positive. Also the blood perfusion is positive; so the differentiation with respect to temperature is positive. The transform is monotonous and nonlinear as it can be seen in Figure 3.6 for different ambient temperatures. Nonlinearity favors the high temperature parts which contain the valuable information about face recognition. 


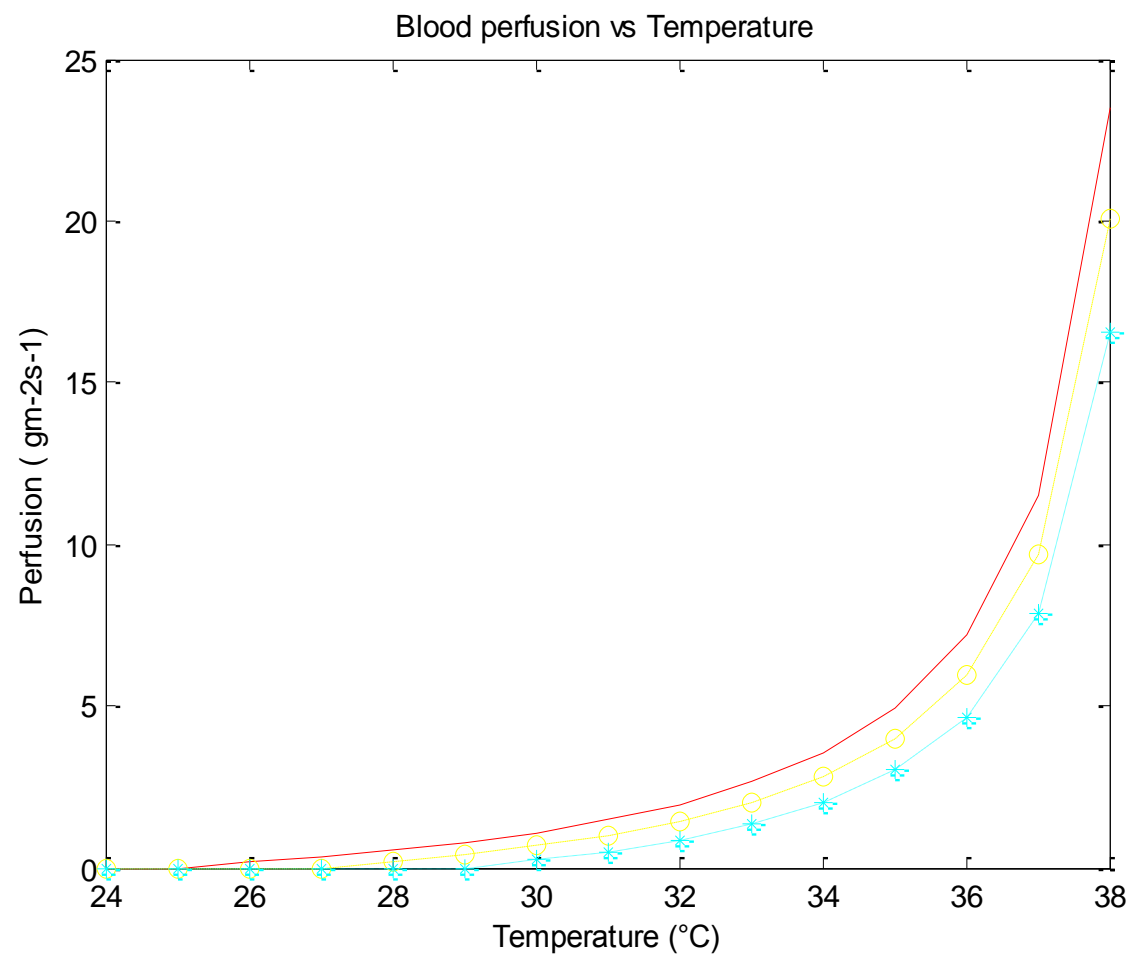

Figure 3.6 Blood perfusion vs temperature graph

A thermal image and its corresponding blood perfusion can be seen in Figure 3.7.

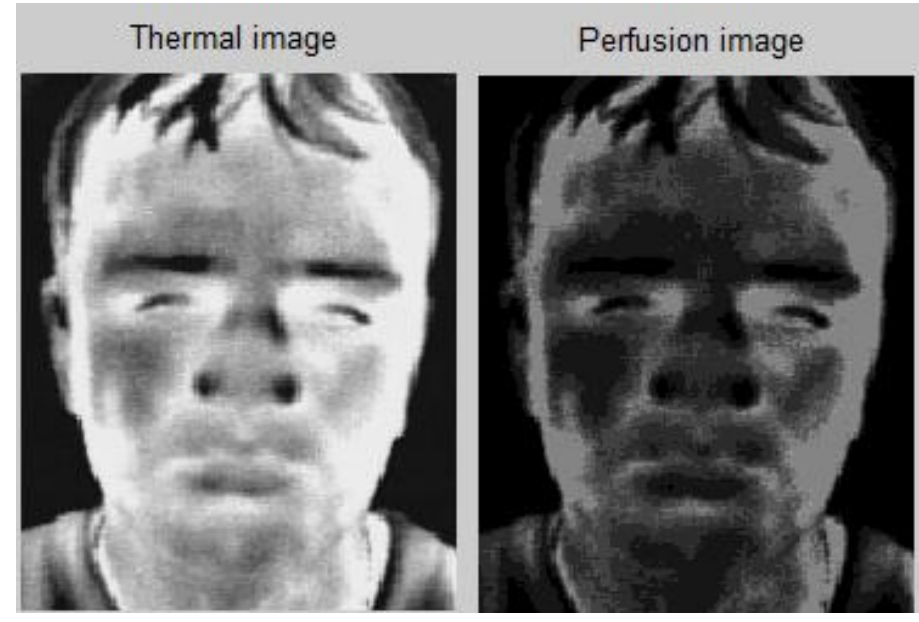

Figure 3.7 Thermal face and corresponding perfusion face 
The transform that is utilized to obtain blood perfusion data can be thought as a preprocessing algorithm, which is followed by an algorithm that is used for classification. In light of this, general flowchart for utilizing blood perfusion method may be expressed as in Figure 3.8.

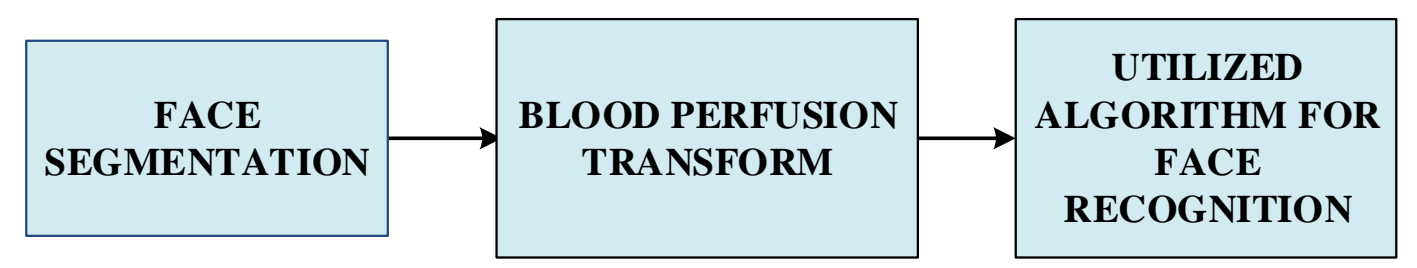

Figure 3.8 General flowchart of the methods that utilize perfusion transform

As stated earlier, the transform utilized to obtain the blood perfusion data does not deteriorate the data for algorithms that utilizes facial features. So both holistic and feature based algorithms may be employed for face recognition. In literature many holistic and feature based algorithms are employed with blood perfusion.

A modified model is proposed to replace the traditional blood perfusion model. $\mathrm{Wu}$ et al. [21] suggests that the terms $\mathrm{H}_{e}, \mathrm{H}_{\mathrm{f}}, \mathrm{H}_{\mathrm{c}}, \mathrm{H}_{\mathrm{m}}$ in the heat influx-outflow equilibrium equation are less significant compared to others. This leads to a quite remarkable simplification of the original transform equation, which is given in Equation (3.21).

$$
w=\frac{\varepsilon \sigma\left(\mathrm{T}^{4}-\mathrm{T}_{\mathrm{e}}^{4}\right)}{\alpha \mathrm{c}_{\mathrm{b}}\left(\mathrm{T}_{\mathrm{a}}-\mathrm{T}\right)}
$$

This modified model achieves very similar results with the original model. In addition, it is seen that this modified approach yields better results for time lapse data which is one of the main obstacles faced in thermal face recognition.

Another new model for blood perfusion is proposed by Xie et al. [77], which is called Penn Equation based perfusion model. It is based on the classical bio-heat 
transfer equation, also known as Pennes Equation. The Pennes equation is given in Equation (3.22).

$$
\rho c \frac{\partial T}{\partial t}=\nabla \cdot(K \nabla T)+W_{b} C_{b}\left(T_{a}-T\right)+q_{m}+q_{r} .
$$

Where $\rho$ is the tissue density, $\mathrm{c}$ is specific heat of the tissue, $\mathrm{T}$ the tissue temperature, $\mathrm{K}$ is the tissue thermal conductivity; $\mathrm{W}_{\mathrm{b}}$ is the blood perfusion rate, $\mathrm{C}_{\mathrm{b}}$ is the blood specific heat, $\mathrm{T}_{\mathrm{a}}$ is the arterial temperature, and $\mathrm{q}_{\mathrm{m}}$ is the tissue metabolic heat generation rate, $\mathrm{q}_{\mathrm{r}}$ is the heat generation rate from ambient environment.

Pennes equation is three dimensional, whereas facial temperature field of thermal images under steady state are two dimensional. Considering two dimensional thermal field of infrared face, $\rho_{c} \frac{\partial T}{\partial t}$ time derivative is zero. There is no heat source so $\mathrm{q}_{\mathrm{r}}$ term is also zero and facial tissues are isotropic. These are stated for steady state conditions. So the Pennes Equation reduces to two dimensions. The resulting equation for $\mathrm{Wb}$ is continuous, whereas facial thermograms are discrete. The equation for $\mathrm{Wb}$ also needs to be discrete. The classic Laplace operator is utilized for this purpose. With further simplification Equation (3.23) is obtained, which is the perfusion transform.

$$
\begin{aligned}
& W_{b}(\mathrm{i}, \mathrm{j}) \\
& =\frac{-K \times \lambda \times\left(\mathrm{T}(\mathrm{i}-1, \mathrm{j})+\left(\mathrm{T}(\mathrm{i}+1, \mathrm{j})+\left(\mathrm{T}(\mathrm{i}, \mathrm{j}-1)+(\mathrm{T}(, \mathrm{j}+1)-4 \mathrm{~T}(\mathrm{i}, \mathrm{j}))-\mathrm{q}_{\mathrm{m}}\right.\right.\right.}{\mathrm{C}_{\mathrm{b}}\left(\mathrm{T}_{\mathrm{a}}-\mathrm{T}\right)} .
\end{aligned}
$$

In this model, every pixel represents a blood perfusion rate. Figure 3.9 illustrates resulting perfusion image obtained from original blood perfusion and Pennes equation based blood perfusion. 

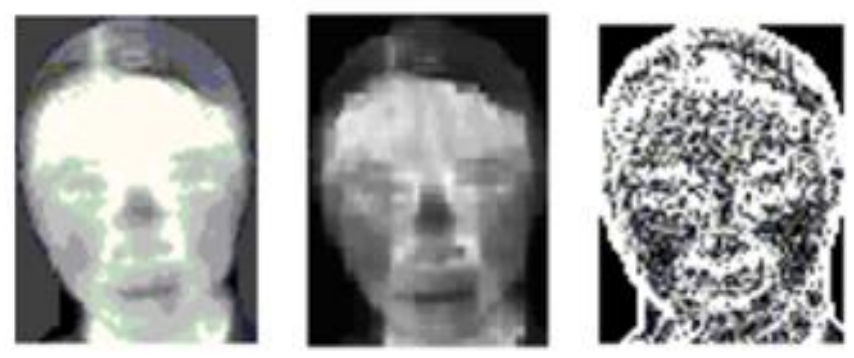

Figure 3.9 Different perfusion models are presented for visual comparison [77]. The image on the left is the original thermal image. The image in the middle is the original blood perfusion image. The image on the right is Pennes blood perfusion image.

When Figure 3.9 is investigated, it is easily notable that Pennes based blood perfusion seem to alter the image. The transform in Pennes based blood perfusion in not a point wise transform. Neighboring pixels affect the result of the transform for a given point. With that information in mind, Pennes blood perfusion model is not chosen in this thesis work.

\subsubsection{Vascular Network Method}

The third method implemented in this thesis work is vascular network method. In this section general principles of the method and significant works are investigated.

Physiology based face recognition is one of the methods that is specifically developed for infrared images. The method is developed to take advantage of the underlying physiological information below the skin. The method first proposed by Buddharaju et al. [47], [69]. It makes use of the fact that the characteristics under the face skin are permanent and the vessels are hard wired. It is noted in this work that the IR cameras have the capability to indirectly image the superficial blood vessels under the skin. The work of P1nar and Govsa [70], which investigated the Superficial Temporal artery and branches, is referred in this paper. P1nar and Govsa 
concluded that the position and the branching points of the superficial temporal artery vary from person to person. In the work of Buddharaju et al. [69] the assumption is made that the same could be told for other vessels. Other than this observed characteristic for superficial temporal artery, it is also noted that the skin complexion and fat composition will also contribute to the uniqueness of vascular network of the face. This idea is based upon the fact that thermal images vary because the radiance is absorbed in different proportions under different skin types and different fat percentages. So it is suggested that even if the two underlying vascular networks are the same, this phenomenon will give the algorithm a distinctive power. It is also stated that this method can be used to discriminate identical twins which the visible spectrum recognition methods fail to do so. In visible spectrum the identical twins look very much alike and it is very hard to discriminate them by their appearances.

The first step in their work is face segmentation. After segmenting the face preprocessing techniques are applied to reduce the noise and highlight edges. This is required because of the weak sigmoid edges that the blood vessels have with the surrounding tissue. This phenomenon is caused by heat diffusion between tissues in contact. This process, called anisotropic diffusion, handles this problem and highlights the edges between different regions. It does so by smoothing in intraregions as opposed to inter-region smoothening. Anisotropic diffusion also helps to reduce the noise caused by hair over the skin. Anisotropic diffusion is expressed mathematically as in Equation (3.24).

$$
\frac{\partial I(\bar{x} \cdot t)}{\partial t}=\nabla(c(\bar{x}, t) \nabla I(\bar{x}, t))
$$

Here $\mathrm{x}$ is spatial dimensions and $\mathrm{t}$ is time. The anisotropic diffusion in discrete form is given in Equation (3.25). 


$$
\begin{aligned}
I_{t+1}(x, y)=I_{t} & +\frac{1}{4}\left(c_{N, t}(x, y) \nabla I_{N, t}(x, y)\right. \\
& +c_{S, t}(x, y) \nabla I_{S, t}(x, y)+c_{E, t}(x, y) \nabla I_{E, t}(x, y) \\
& \left.+c_{W, t}(x, y) \nabla I_{W, t}(x, y)\right) .
\end{aligned}
$$

As one can see there are four coefficients and they are calculated in the same manner that Equation (3.26) illustrates for the north direction coefficient. $I_{N, t}$ term in Equation (3.26) is given in Equation (3.27).

$$
\begin{gathered}
c_{N, t}(\mathrm{x}, \mathrm{y})=\exp \left(\frac{-\nabla I_{N, t}^{2}(x, y)}{\mathrm{k}^{2}}\right), \\
I_{N, t}=I_{t}(x, y+1)-I_{t}(x, y) .
\end{gathered}
$$

The effect of this filtering can be seen in Figure 3.10. As one can note the noise is removed significantly and the boundaries are enhanced.
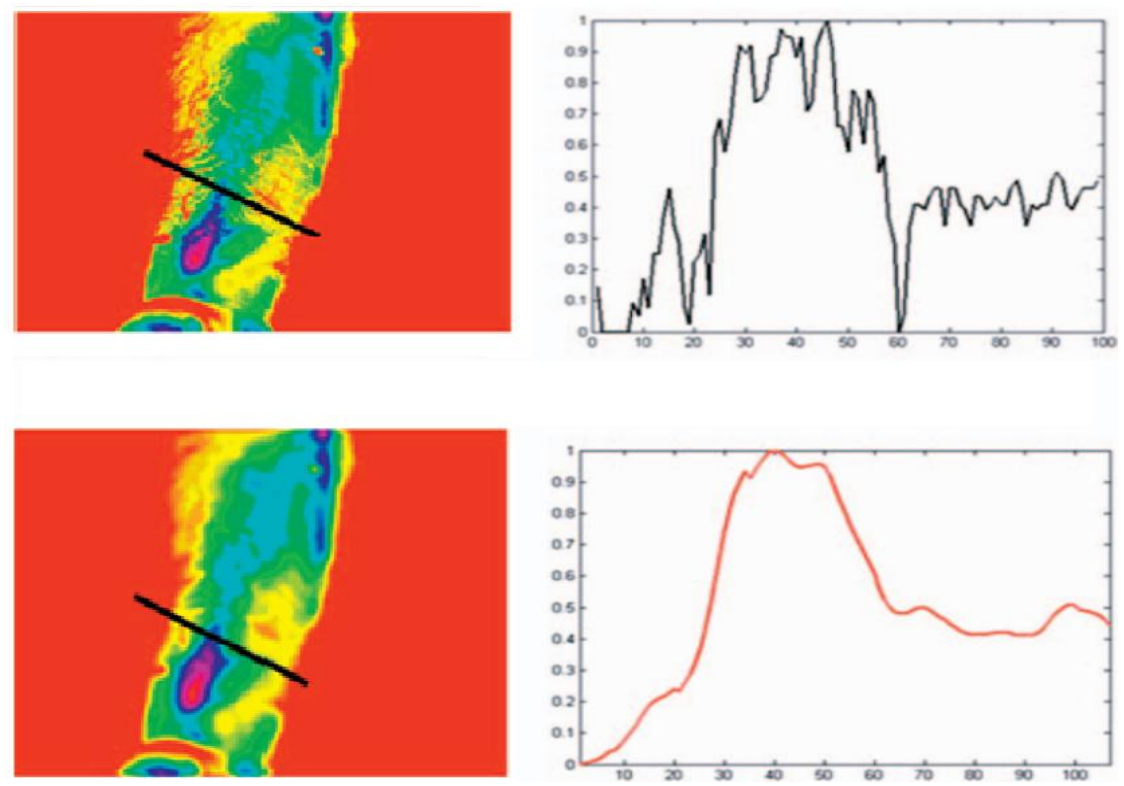

Figure 3.10 The effect of anisotropic diffusion. The top left image is a thermal wrist image and top right image is the corresponding line profile shown as a black line. The bottom left image is the resulting image after anisotropic diffusion and the bottom right image is its corresponding line profile. [69] 
The next step in the algorithm is to segment the vascular network. This is done by a series of morphological operations. The operation is called white top hat operation which is a series of dilation and erosion operations that is employed to enhance brighter objects, in this case superficial vessels. First the image is "opened" and it is subtracted from the original image. The resulting image is the segmented vascular network. The mathematical representation of top hat segmentation is given in Equation (3.28). The visual results of the white top hat segmentation can be seen in Figure 3.11. This figure is not taken from Buddharaju' work, it is the implementation of top hat transformation in this thesis.

$$
\begin{gathered}
I_{\text {open }}=(I \ominus S) \oplus S, \\
I_{\text {top }}=I-I_{\text {open }} .
\end{gathered}
$$
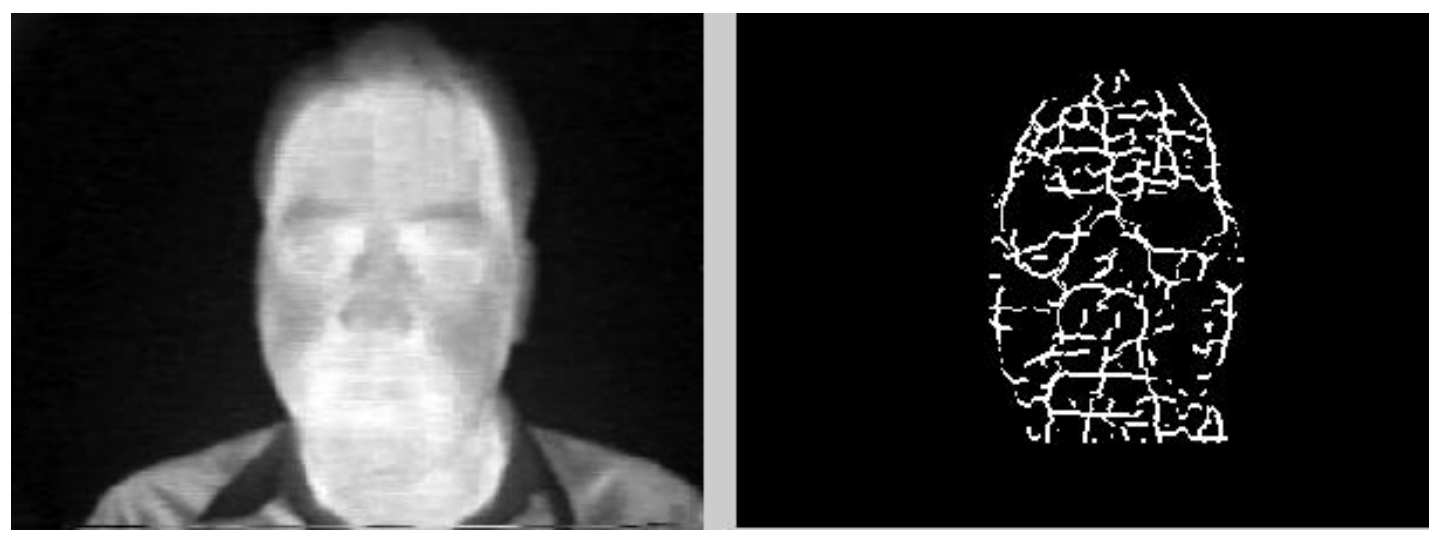

Figure 3.11 Original segmented image and extracted blood vessels with top hat operation

After the segmentation of the vascular network, thermal minutia points are extracted. First the orientation function is decided, which is the angle between the blood vessel contour and the horizontal axis. Following that the vascular network is thinned to one pixel thickness. If the pixel is in the thinned vascular network, it is represented with a one otherwise with a zero. If the point is a branching point it is called a thermal minutia point. The last step is the removal of the thermal minutia points that belong to trivially short branches and ones that belong to a cluster. Thermal minutia 
points are obtained for every image in gallery and they are saved. Given an image the thermal minutia points extracted from that image is compared with the ones that are saved. The classification is made through a feature vector that is of the form given in Equation (3.29). Equations (3.30), (3.31) and (3.32) describe how each parameter is calculated.

$$
\begin{gathered}
L_{M}=\left(\left(d_{1}, \varphi_{1}, \vartheta_{1}\right),\left(d_{2}, \varphi_{2}, \vartheta_{2}\right), \ldots . .\left(d_{N}, \varphi_{N}, \vartheta_{N}\right), \Psi\right) \\
d_{n}=\sqrt{\left(x_{n}-x\right)^{2}+\left(y_{n}-y\right)^{2}} \\
\varphi_{n}=\operatorname{diff}\left(\Psi_{n}, \Psi\right) \\
\vartheta_{n}=\operatorname{diff}\left(\arctan \left(\frac{y_{n}-y}{x_{n}-x}\right), \Psi\right)
\end{gathered}
$$

Extracted minutia points are in the form of $\mathrm{M}(\mathrm{x}, \mathrm{y}, \Psi)$, where $\Psi$ is orientation. "diff" operation here corresponds to difference between angles scaled to $(0,2 \Pi)$. If the obtained feature vector and orientation difference for a thermal minutia point is below some threshold; thermal minute points are matched. Final decision made on the image depends on the score that is calculated in the manner given in Equation (3.33) where NUM represents the number of minutia points.

$$
\text { Score }=\frac{N U M_{\text {match }}}{\max \left(N U M_{\text {test }}, N U M_{\text {database }}\right)} .
$$

This work showed that this approach has discriminative power and in fact it is a promising method. Promising results are obtained and the method outperformed 
PCA. In the Houston University Database a maximum first rank recognition rate of $84 \%$ is achieved for frontal images and $86 \%$ for arbitrary pose.

In their later work Buddharaju and Pavlidis [71] improved this algorithm. A post processing step and a new matching algorithm is proposed in order to overcome some of the weaknesses that the earlier version encountered. Post processing step tackles with fake vascular networks that the white top hat produces. The new matching algorithm tackles with the problems that the old one had in existence of deformations caused by pose and expression variance. After thinning the vascular network, in the new version a parallelogram is considered for each pixel belonging to vascular network. $\mathrm{K}$-means clustering is applied with $\mathrm{K}$ equal to 2, to the pixels belonging to that parallelogram. A pixel is called and outliner, if the difference between the centers of each cluster is larger than 1.5. Branches are removed if half of the pixels belonging to that branch are outliners. Bootstrap iterative closest point algorithm is employed for classification and a pose estimation step is utilized to decide the appropriate threshold value to be used by iterative closest point algorithm under a certain pose. The new algorithm outperformed the old one and PCA.

A step by step flowchart for the new algorithm proposed by Buddharaju and Pavlidis [71] is given in Figure 3.12.

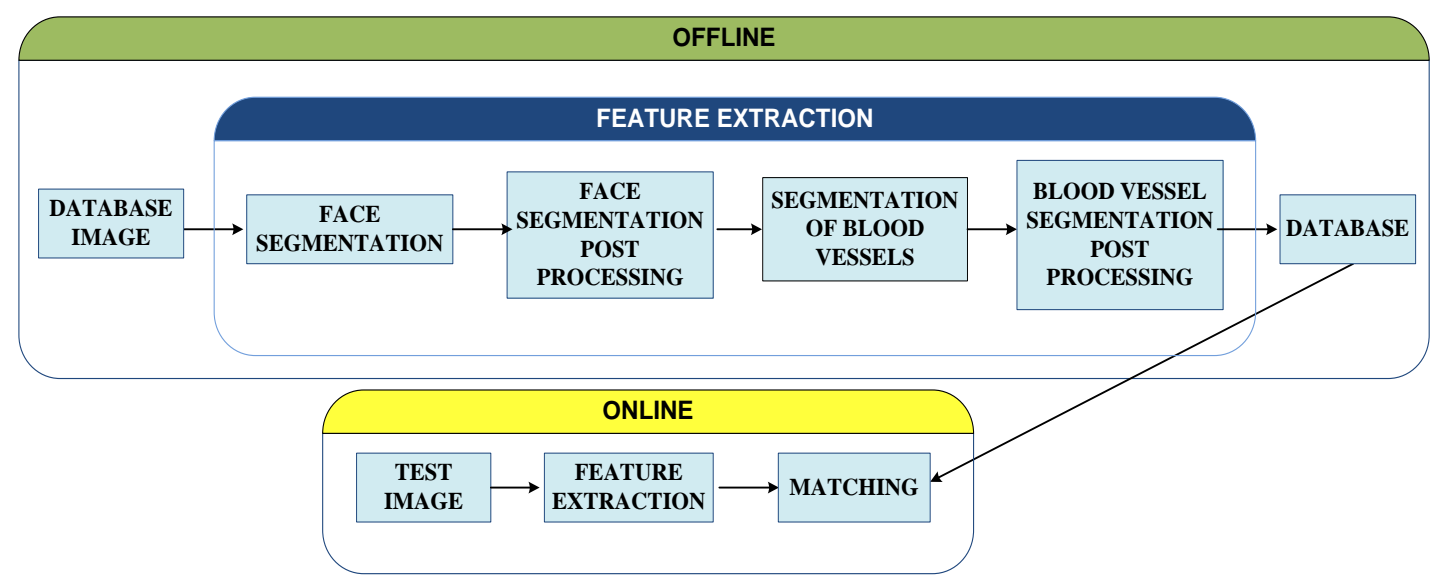

Figure 3.12 Flowchart for the improved algorithm proposed by Buddharaju and Pavlidis [71] 
More recently Ghiass et al. [72], [73] took a different approach in obtaining the vascular network. The method is called vesselness filter, which is introduced by Frangi et al. [74] for the utilization on 3D MRI data. The motive for this approach was to overcome the scale problem that is encountered with the traditional vascular network segmentation method. Vesselness filter is given below in Equation (3.34). $\mathrm{R}_{\mathrm{A}}$ and $\mathrm{S}$ parameter are given in Equation (3.35). $\lambda 1$ and $\lambda 2$ are eigenvalues of the Hessien matrix computed at a certain image locus and a scale. $\left|\lambda_{1}\right|<\left|\lambda_{2}\right|$ is assumed. $\beta$ and $\mathrm{c}$ are parameters that control sensitivity of $\mathrm{R}_{\mathrm{A}}$ and $\mathrm{S}$.

$$
\begin{gathered}
V(s)= \begin{cases}0 & \text { if } \lambda_{2}>0 \\
\left(1-e^{\frac{R_{A}}{2 \beta^{2}}}\right) \times\left(1-e^{\frac{S}{2 c^{2}}}\right) & \text { otherwise. }\end{cases} \\
\left.R_{A}=\frac{\left|\lambda_{1}\right|}{\left|\lambda_{2}\right|} \text { and } S=\sqrt{\left(\lambda_{1}{ }^{2}+\right.} \lambda_{2}{ }^{2}\right) .
\end{gathered}
$$

If this process is applied to different scales, the vesselness response is taken as the maximum result obtained across those scales. For the matching part utilizing a similarity function is proposed and it is calculated as in the Equation (3.36) where $I_{n 1}$ and $I_{n 2}$ are vesselness responses and the terms with the hats are their mean values respectively.

$$
p\left(\mathrm{I}_{\mathrm{n} 1}, \mathrm{I}_{\mathrm{n} 2}\right)=\frac{\sum_{\mathrm{i}, \mathrm{j}}\left(\mathrm{I}_{\mathrm{n} 1}(\mathrm{i}, \mathrm{j})-\overline{\mathrm{I}}_{\mathrm{n} 1}\right)\left(\mathrm{I}_{\mathrm{n} 2}(\mathrm{i}, \mathrm{j})-\overline{\mathrm{I}}_{\mathrm{n} 2}\right)}{\sqrt{\sum_{\mathrm{i}, \mathrm{j}}\left(\mathrm{I}_{\mathrm{n} 1}(\mathrm{i}, \mathrm{j})-\overline{\mathrm{I}}_{\mathrm{n} 1}\right)^{2} * \sum_{\mathrm{i}, \mathrm{j}}\left(\mathrm{I}_{\mathrm{n} 2}(\mathrm{i}, \mathrm{j})-\overline{\mathrm{I}}_{\mathrm{n} 2}\right)^{2}}} .
$$

The utilization of confidence levels is also proposed for the representation of the vascular network. Confidence level is a number in the interval between one and zero rather than binary representation. In binary representation one pixel is either a part of the vascular network or not. New representation offers more flexibility. This method proved to be effective, as better results are obtained with scale changes. Also 
the use of active appearance model is proposed for producing synthetic images to overcome deformations caused by change in poses. The reported results are impressive. This method outperformed both methods proposed by Buddharaju et al. [69], [71]. However it cannot be told that the improvement is because of the vesselness approach or it is because of the use of AAM, preprocessing and confidence levels. It cannot be concluded, other than different scale images, vesselness approach is more effective than vascular network approach.

In their later work Ghiass et al. [73] utilized an ensemble of AAM fitted for the specific pose and facial appearances.

Another work on vascular networks is proposed by Cho et al. [75]. It is based on the approach that Buddharaju proposed. LWIR spectrum band is utilized. As a part of the enhancement scheme, first median filters are employed. Then the image is processed linearly so that the image histogram covers all values in grayscale levels. The next step is to segment the face and first sobel edge detector is utilized. This enhances major edges. The latter step is to employ morphological operations to remove small artifacts and connect major edges. This leads to a closed shape, which is then flood filled to obtain the mask. That mask is multiplied with the original image to obtain the segmented face. After face segmentation the next step in the algorithm is feature extraction. White top hat operation is used to obtain the vascular network. A post processing step is employed so that the false branches are removed. Deciding the bifurcation points, like the ones in fingerprint, is next step. Bifurcation points are the branching points in the vascular network. The spatial positions of the bifurcation points constitute a feature vector for the use of matching step. For matching step Modified Hausdorff Distance (MHD) is utilized. MHD investigates the spatial relation between two sets without forming a one to one correspondence. MHD is also more robust to outliner pixels.

Three images are selected from the training data and MHD's of those three images to the test image are calculated. The MHD to consider for matching is the average of 
those three distances. The smaller the distance is, the higher the degree of similarity is between two images. The authors utilized this algorithm for verification purposes. With the aforementioned method an equal error rate of 18 percent is obtained. Then two more coordinates are appended to that feature vector, namely $\mathrm{x}$ and $\mathrm{y}$ coordinates of the center of mass of pixels that belong to face. This improved the performance of the system significantly with an equal error rate of $6.7 \%$. A false rejection rate of 0.101 and a false acceptance rate of 0.012 is also reported. 


\section{CHAPTER 4}

\section{ALGORITHMS FOR INFRARED FACE RECOGNITION}

In this thesis work, recognition task is under consideration. Four different methods are implemented concerning infrared face recognition. The first one of those four methods is PCA. It is a well established method that is often utilized for performance comparison. It is utilized as the base method in this work. The second method is the blood perfusion method. It is a hot topic in infrared face recognition. The recent works utilize this method since it is a method specifically designed to overcome some of the limitations experienced by thermal face recognition systems. In second method blood perfusion is utilized in conjunction with PCA. The third method is vascular network method. It is also one of the most popular and promising methods in infrared face recognition. This method utilizes physiological information under the skin. This makes the method more robust as those information are observed to be unique for each person and do not change according to surrounding conditions. Even the growth of the vessels is predictable. The fourth method is proposed first time in this thesis work. The method is based on utilizing vascular network method with the blood perfusion transform. The motivation was to investigate whether the advantages of blood perfusion are present when it is utilized with vascular network method.

\subsection{PCA Method Implementation}

The flowchart of the algorithm is given in Figure 4.1. 


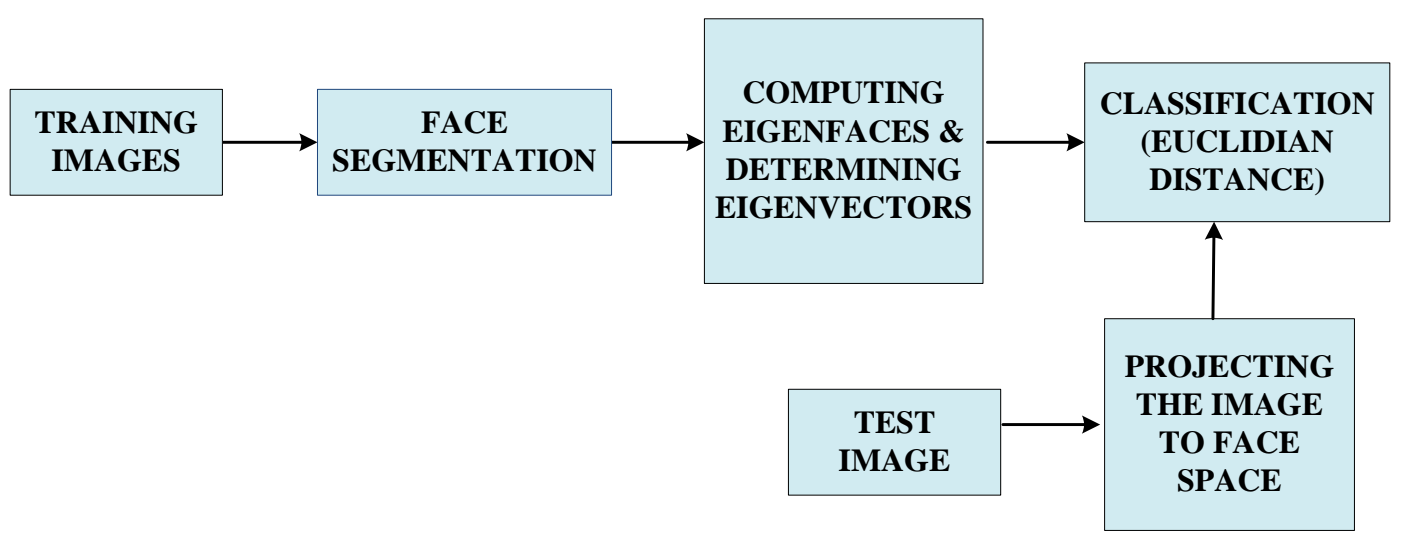

Figure 4.1 Flowchart of the PCA method described in the thesis work

In our implementation the aforementioned procedure for PCA is followed. An image is taken for each subject. First the images are rearranged as a column vector. Those column vectors are utilized together to form a vector that contains every image. Average face image is calculated from the column vectors obtained from the images. Mean vector is subtracted from the unified matrix. As explained in general PCA section, computational requirement of calculating the eigenvalues of the $\delta^{\mathrm{T}} \delta$ matrix is much less compared to calculating the eigenvalues of $\left(\delta \delta^{\mathrm{T}}\right)$ matrix. The obtained eigenvalues are sufficient as they are linearly related with the original eigenvalues. Next step is to decide the number of sufficient eigenvectors. In order to decide the significant eigenvectors to describe the training set of images appropriately, Kaiser's rule is employed. It is one of the most frequently used techniques in deciding the principal components. The rule is fairly simple. If the eigenvalue that corresponds to the eigenvector is greater than one, that eigenvector is considered to be a significant eigenvector. Given a test image, the image is first projected to the face space. Than in deciding which class does that image belongs to, Euclidian Distance is utilized. The image class with the smallest distance is returned as the recognized face. 


\subsection{PCA Method Applied with Blood Perfusion Transform}

The flowchart of the algorithm is given in Figure 4.2.

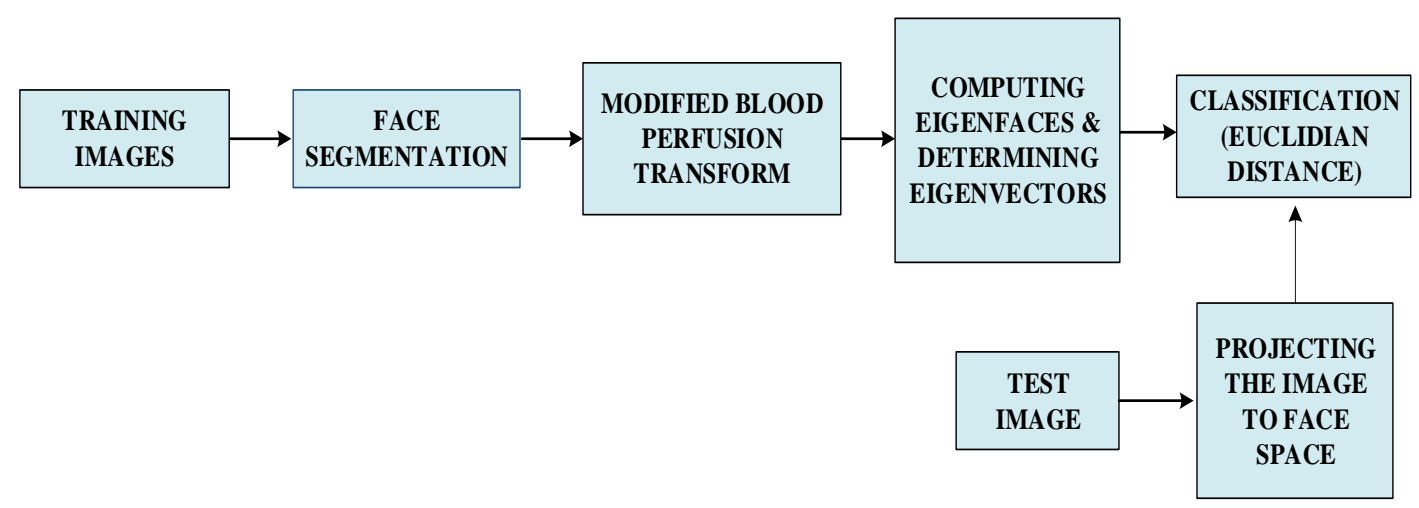

Figure 4.2 Flowchart of PCA method applied with blood perfusion transform

The second method implemented in this thesis work is blood perfusion. The modified blood perfusion model is preferred for several reasons. The mathematical representation of the transform was given in Equation (3.21) in the previous chapter. Modified blood perfusion model offers the same set of advantages with the original blood perfusion model. Modified blood perfusion is significantly less expensive in terms of computation requirements. Also modified blood perfusion model does not alter the image unlike the Pennes blood perfusion model where the thermal image is significantly altered and the resulting image looks dotted. This is preferable in the context that later in the thesis work a method, which utilizes the underlying vessel structure will be proposed on top of this model. The method relies on applying vascular network method to modified blood perfusion transform of the image. This method is introduced for the first time in this thesis work and will be explained in the following sections of this chapter. So it is important to preserve the facial structure intact.

The second method, namely the PCA method applied with blood perfusion transform, works as follows. Given an image, modified blood perfusion transform is 
calculated. For every subject an image is taken to form training image gallery. The resulting data after applying modified blood perfusion transform is called blood perfusion transform of the thermal data. Training gallery images' perfusion transform are fed to PCA in order to calculate the eigenvalues of the covariance matrix. Kaiser's rule is applied in order to decide the significant eigenvectors. When a test image is presented, first modified blood perfusion is calculated for this test image. Test image's projection to the perfusion face space is calculated. Then in order to identify the class of the image Euclidian distance is utilized. The training image with the smallest distance to the test image is returned as the recognized face. Note that PCA approach in this method is the same with the one that is used in the first method. The only difference between the first and the second method is that in this second method PCA is applied to blood perfusion transform of the thermal image rather than thermal image itself.

\subsection{Vascular Network Method Implementation}

In this section the third method implemented in this thesis work, which is the vascular network method, is described. The vascular network method implemented in this thesis follows the work of Cho et al. [75] with few differences. Utilized face segmentation methods are different. Also in this thesis work recognition task is under consideration, unlike the work of Cho et al.[75] which considers the authentication task. Three different feature vector approaches are investigated for this method. Those feature vector approaches are referred as A, B and C. The flowcharts of all three feature vector approaches for this method are given in Figure 4.3, Figure 4.4 and Figure 4.5. 


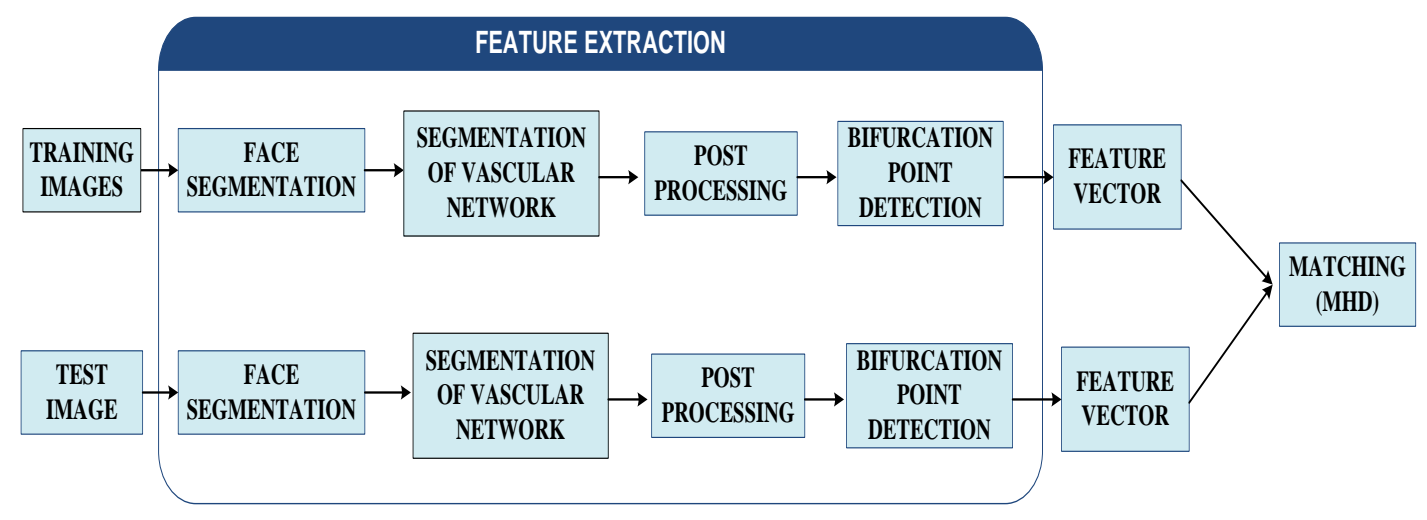

Figure 4.3 Flowchart of the vascular network method feature vector approach A

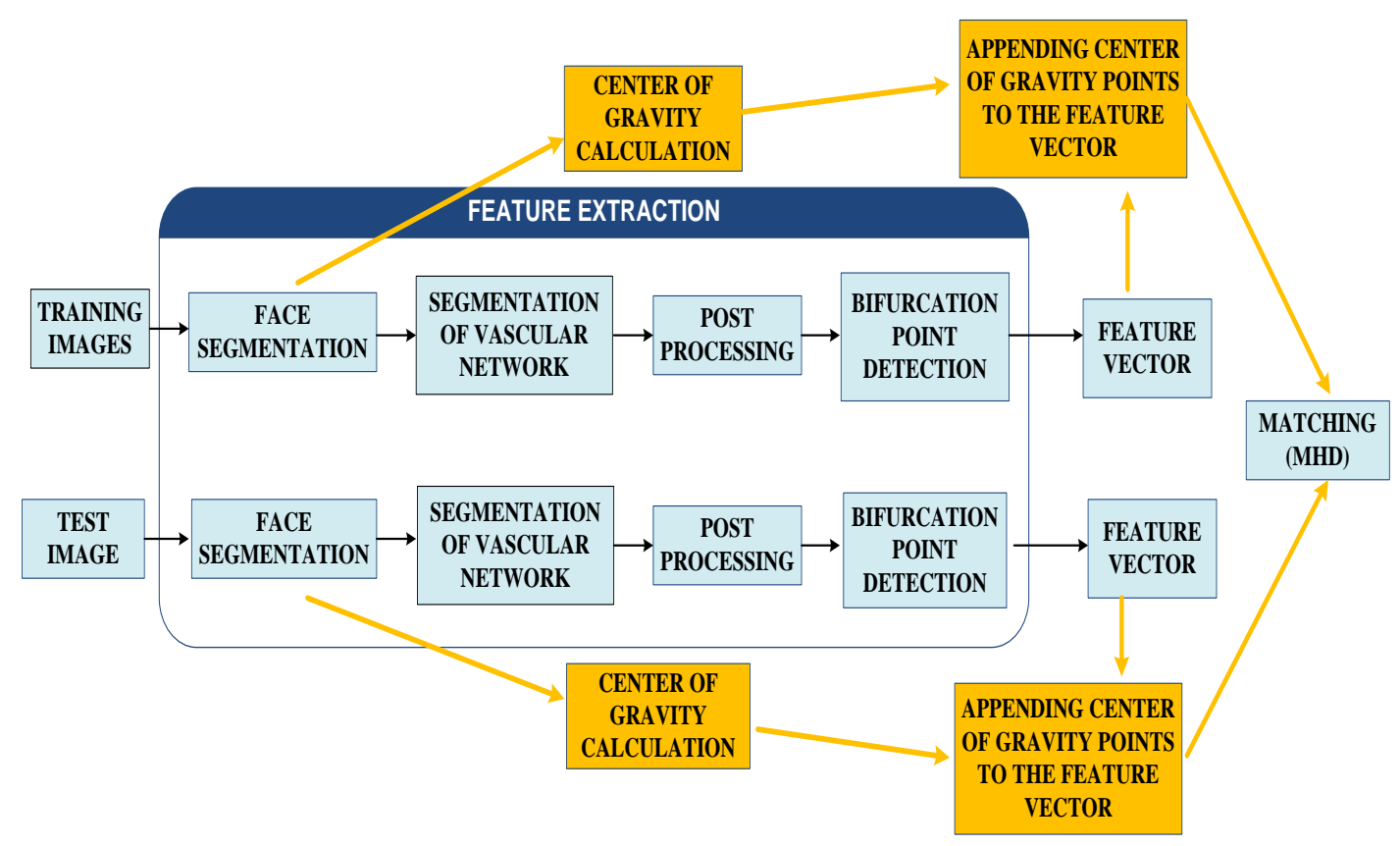

Figure 4.4 Flowchart of the vascular network method feature vector approach B 


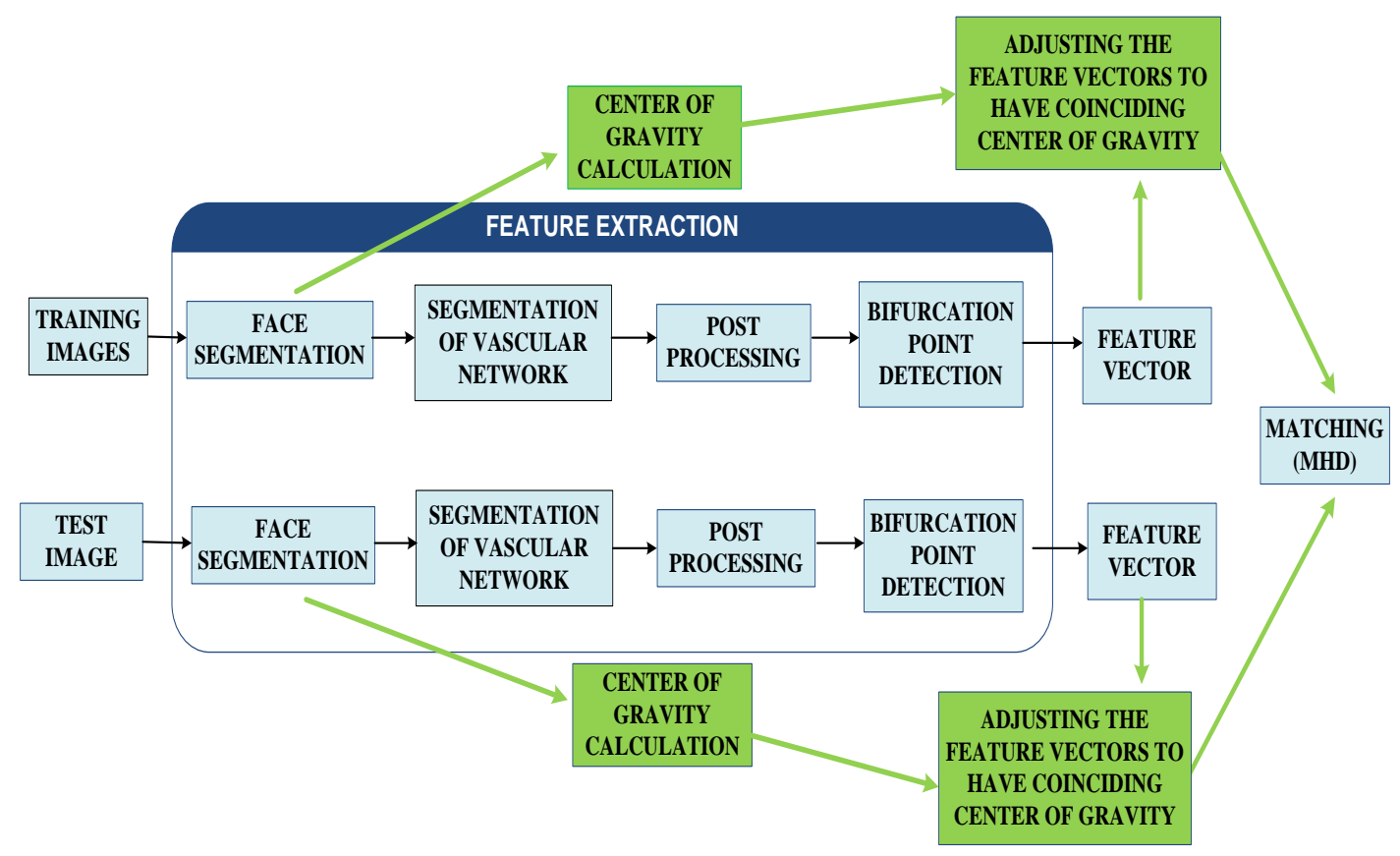

Figure 4.5 Flowchart of the vascular network method with proposed feature vector approach $\mathrm{C}$

The first step in the algorithm is face segmentation. The utilized segmentation method is explained thoroughly in chapter 3. After face segmentation, the latter steps follow the work of Cho et al. [75]. White top hat operation is utilized to segment the vascular network. It is the preferred algorithm in many of the works including the Buddharaju's original vascular network method. As explained in former chapter, it is a series of morphological operations. A 3 by 3 structuring element is utilized. Mathematical representation of the procedure can be found in Equation (3.28).

The vascular network is obtained at this step but it requires further processing. An example vascular network can be seen in Figure 4.6. 


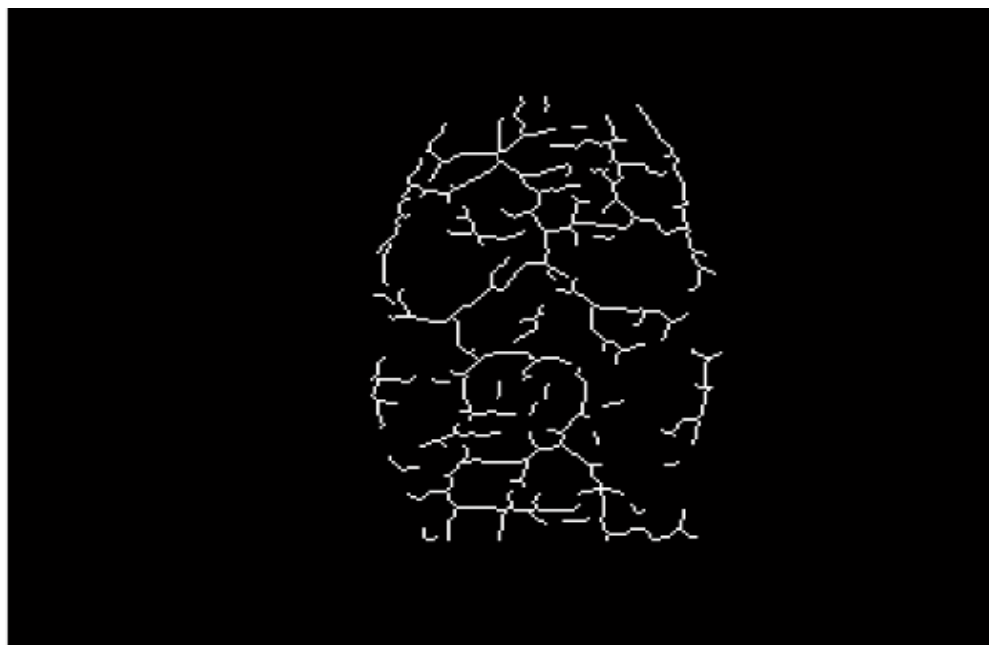

Figure 4.6 An example vascular network

Local orientation of each point in vascular network is calculated. The obtained vascular network is thinned to one pixel thickness. This is done in order to find the exact branching points. The short spurious branches are removed. The branches in the vascular network that contain less than ten pixels are removed. Bifurcation points are extracted from the vascular network. Bifurcation points are the branching points of the vascular network that are found by investigating the 8 neighborhood around a point belonging to vascular network. The points in the vascular network are represented with one and the other pixels with zero. If the sum of the pixels in the 8 neighborhood for a pixel in the vascular network is bigger than two, that point is called a bifurcation point. For matching images MHD is utilized. The mathematical representation is given in Equation (4.1), where $\mathrm{M}^{\mathrm{p}}$ and $\mathrm{T}^{\mathrm{p}}$ are sets of bifurcation points and $\mathrm{N}_{\mathrm{m}}{ }^{\mathrm{p}}$ is the number of members in the set $\mathrm{M}^{\mathrm{p}}$. Three templates are selected from the training images and MHD's are calculated. The MHD to consider for matching is the average of those three distances. The distance with the training images are calculated for a given test image. The smaller the distance is the higher the degree of similarity is between two images. The person with the smallest calculated distance is returned as the recognized person. 


$$
\begin{gathered}
\mathrm{h}\left(\mathrm{M}^{\mathrm{P}}, \mathrm{T}^{\mathrm{P}}\right)=\frac{1}{N_{m}^{P}} \sum_{\begin{array}{r}
m_{i}^{P} \in M^{P} \\
t_{i}^{P} \in T^{P}
\end{array}} \min \left|m_{i}^{p}-t_{i}^{p}\right|, \\
\mathrm{H}\left(\mathrm{M}^{\mathrm{P}}, \mathrm{T}^{\mathrm{P}}\right)=\max \left(h\left(M^{P}, T^{P}\right), h\left(T^{P}, M^{P}\right)\right) .
\end{gathered}
$$

Three different feature vector approaches will be presented for this method. First one (A) directly utilizes a feature vector that only contains spatial positions of the bifurcation points. The feature vector $\mathrm{V}$ has the form given in Equation (4.2), where $\mathrm{N}$ is the number of bifurcation points of that facial image.

$$
V=\left(\left(x_{1}, y_{1}\right),\left(x_{2}, y_{2}\right), \ldots\left(x_{N}, y_{N}\right)\right)
$$

Modified Hausdorff Distance between such feature vectors is utilized for classification.

In the second feature vector approach (B), a different feature vector is utilized. The new feature vector also contains the spatial positions of the bifurcation points, like the first one. In addition, the coordinates of the center of gravity of the face is also appended to this vector. The new feature vector has the form given in Equation (4.3), where $\mathrm{N}$ is the number of bifurcation points of that facial image; $\mathrm{x}_{\mathrm{g}}$ and $\mathrm{y}_{\mathrm{g}}$ are geographical gravity centers.

$$
V=\left(\left(x_{1}, y_{1}, x_{g}, y_{g}\right),\left(x_{2}, y_{2}, x_{g}, y_{g}\right), \ldots\left(x_{N}, y_{N}, x_{g}, y_{g}\right)\right)
$$

$\mathrm{x}_{\mathrm{g}}$ and $\mathrm{y}_{\mathrm{g}}$ are the mean of spatial coordinates of the pixels belonging to the segmented face regions. Equation (4.4) illustrates how those points are calculated.

$$
\begin{aligned}
& x_{g}=\frac{1}{N} \sum_{I\left(x_{i}, y_{i}\right) \in \text { face regions }} x_{i}, \\
& y_{g}=\frac{1}{N} \sum_{I\left(x_{i}, y_{i}\right) \in \text { face regions }} y_{i} .
\end{aligned}
$$


As in the first feature vector approach feature vectors are utilized with MHD for classification. Second feature vector approach, like the first feature vector approach, is proposed in the work of Cho et al. [75].

A third feature vector approach (C) is proposed in this thesis work. The addition of center of mass coordinates increases the recognition rates. However, it is not a very useful property. In fact those terms dominate the other terms and consequently the overall result. It increases the recognition rate because the images of the same person in the same session tend to have the same center of gravity. Consider an image and shift its center coordinates. It is fairly possible the image recognized will not be the correct image because of the shift in the center coordinates. So a new feature vector approach is proposed for the first time in this thesis work. This feature vector is formed by bifurcation points only but first those points are adjusted. The gravity center of the images are mapped to the same point and every bifurcation point are adjusted accordingly. Consider two images represented with letters A, B. The corresponding bifurcation points are given in Equation (4.5).

$$
\begin{aligned}
V^{A} & =\left\{\left(x_{1}^{A}, y_{1}^{A}\right),\left(x_{2}^{A}, y_{2}^{A}\right), \ldots\left(x_{N}^{A}, y_{N}^{A}\right)\right\} \text { with gravity center at }\left(x_{g}^{A}, y_{g}^{A}\right), \\
V^{B} & =\left\{\left(x_{1}^{B}, y_{1}^{B}\right),\left(x_{2}^{B}, y_{2}^{B}\right), \ldots\left(x_{N}^{B}, y_{N}^{B}\right)\right\} \text { with gravity center at }\left(x_{g}^{B}, y_{g}^{B}\right) .
\end{aligned}
$$

The bifurcation points in image $\mathrm{B}$ will be shifted $\left(\mathrm{x}_{\mathrm{g}}^{\mathrm{A}}-\mathrm{x}_{\mathrm{g}}{ }^{\mathrm{B}}, \mathrm{y}_{\mathrm{g}}{ }^{\mathrm{A}}-\mathrm{y}_{\mathrm{g}}{ }^{\mathrm{B}}\right)$ pixels. This is done in order to map the gravity centers of the two images to the same point. The corresponding feature vector for image B can be seen in the Equation (4.6).

$$
\begin{gathered}
V^{B}=\left\{\left(x_{1}^{B}-x_{g}^{B}+x_{g}^{A}, y_{1}^{B}-y_{g}^{B}+y_{g}^{A}\right),\left(x_{2}^{B}-x_{g}^{B}+x_{g}^{A}, y_{2}^{B}-y_{g}^{B}\right.\right. \\
\left.\left.+y_{g}^{A}\right), \ldots\left(x_{\mathrm{N}}^{B}-x_{g}^{B}+x_{g}^{A}, y_{\mathrm{N}}^{B}-y_{g}^{B}+y_{g}^{A}\right)\right\} \\
\text { with gravity center at }\left(x_{g}^{A}, y_{g}^{A}\right) .
\end{gathered}
$$

This is a better way to compare two vascular networks, since in this case two vascular networks will be overlapping with coinciding centers. So the distances obtained in this case are much more accurate than they are in the other two feature vector approaches of this method. Feature vector approach $\mathrm{C}$ significantly 
outperforms the feature vector approach A. The feature vector approach B outperforms the feature vector approach $\mathrm{C}$; but as discussed earlier the reason is not that it possess more discriminative power; but merely the fact that the images for the same person has very similar center of gravity points for the same session data. The center of gravity terms dominates in the hausdorff distance calculation; the bifurcation points in the equation lose their significance. In terms of discriminative power this is not a desired property.

\subsection{Vascular Network Method Applied to Blood Perfusion Transform of the Thermal Image}

The fourth method in this thesis work, to best of our knowledge, is proposed first time in this thesis work. The method relies on vascular network method for classification. The application of vascular network technique to the blood perfusion transform of the thermal image is investigated in this method. This has not been done previously in any work before. Again three different feature vector approaches are investigated and they are referred as A, B and C. The flowcharts of all three feature vector approaches for the proposed method are given in Figure 4.7, Figure 4.8 and Figure 4.9.

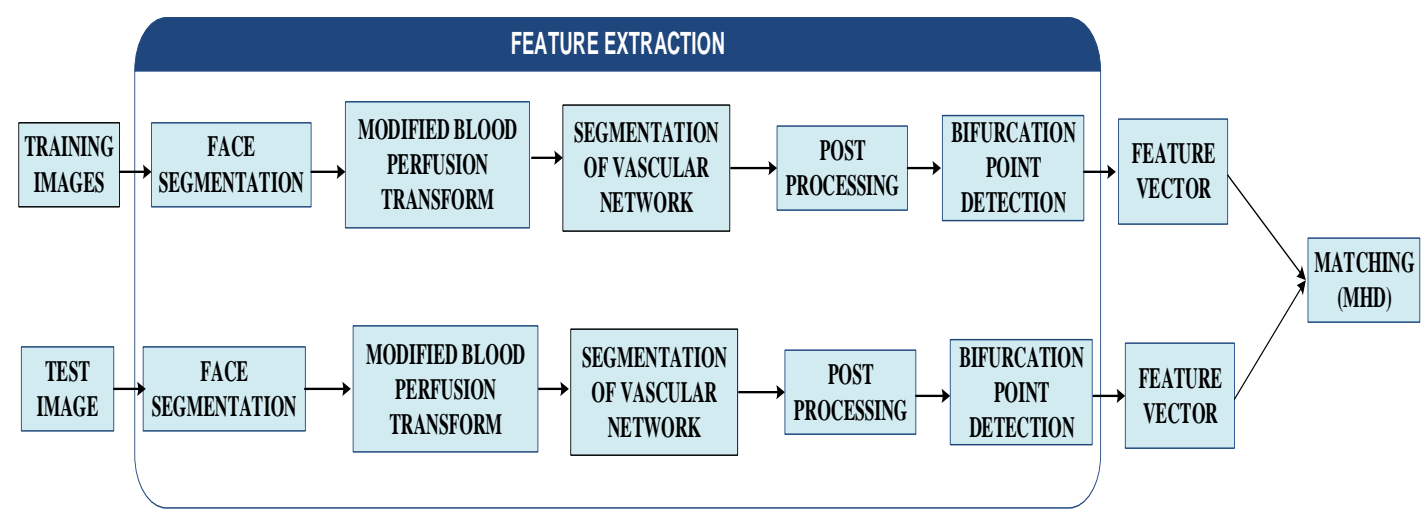

Figure 4.7 Flowchart of the proposed method feature vector approach A 


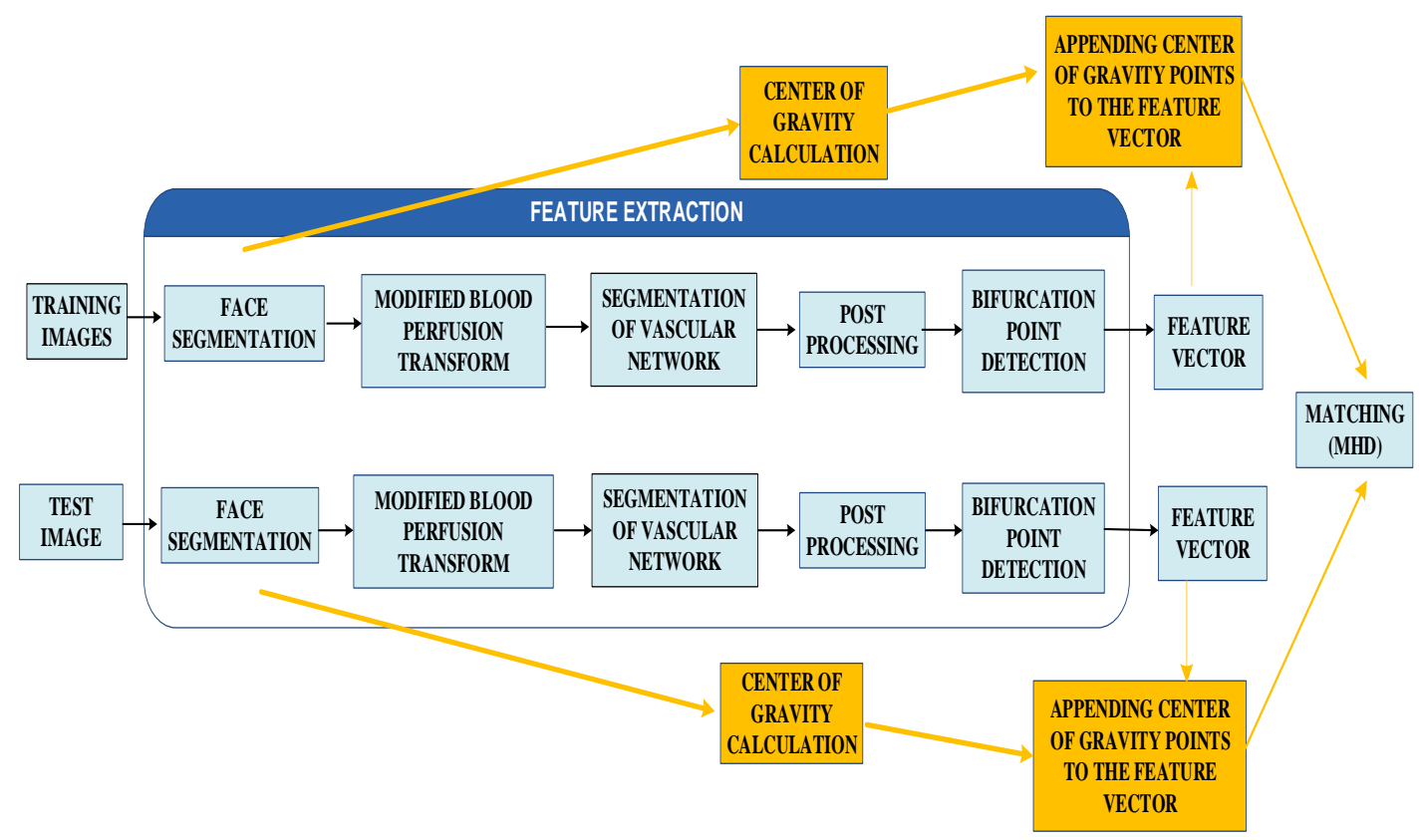

Figure 4.8 Flowchart of the proposed method feature vector approach B

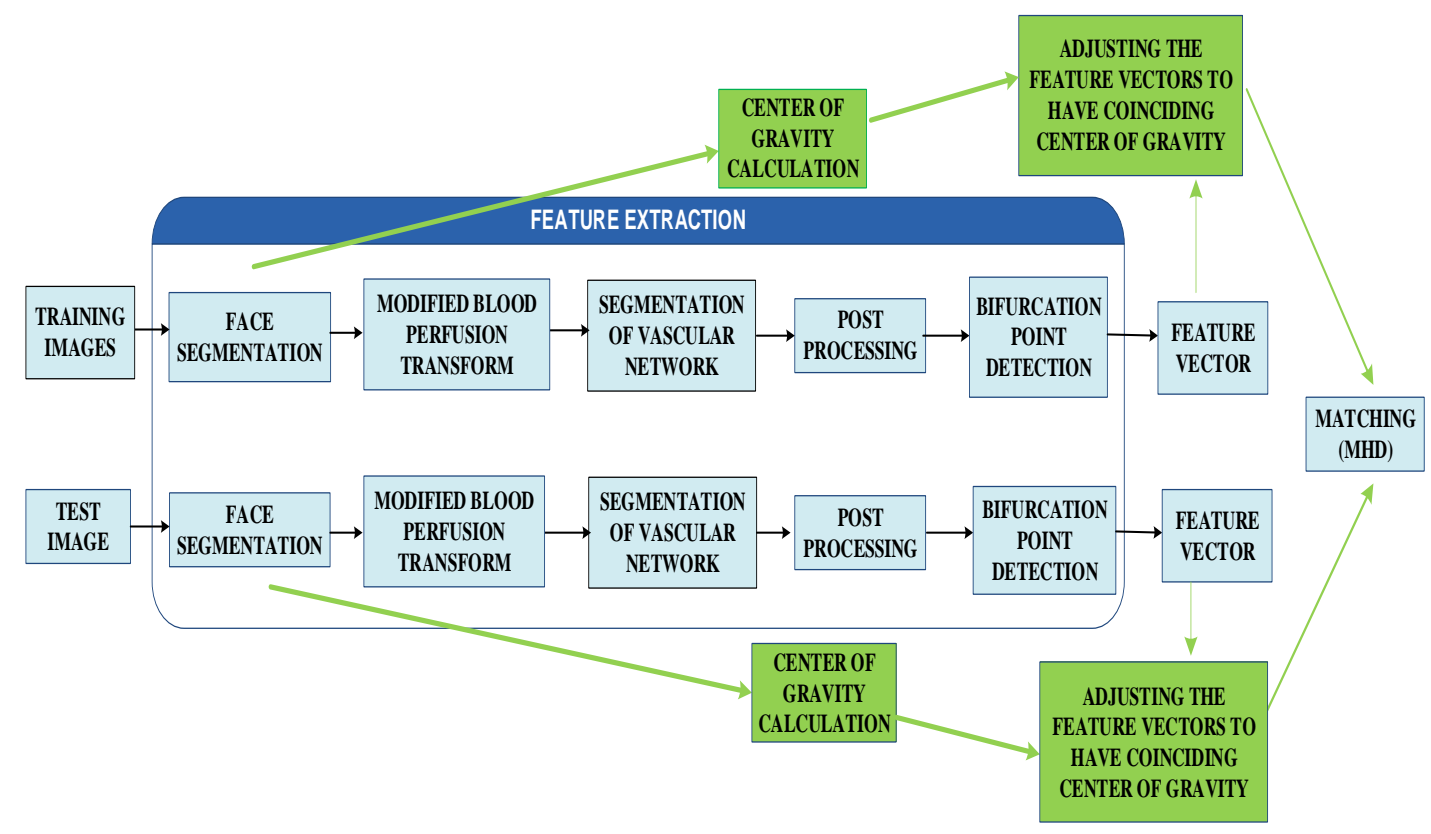

Figure 4.9 Flowchart of the proposed method feature vector approach $\mathrm{C}$ 
In theory the application of vascular network method to perfusion transform of the thermal data is feasible. As stated in the blood perfusion section in chapter 3, blood perfusion highlights the high temperature regions in the face. This is achieved as a result of the nonlinear nature of the transform. The transform suppresses contrast for low temperature regions and enhances them for high contrast regions. Blood vessels have higher temperature than the surrounding tissues. This leads to a small but recognizable contrast with the surrounding tissues. Vascular network is based on this principle and blood perfusion transform is shown to increase the contrast in high temperature regions. Considering this two characteristics, these methods seem to be in line with each other.

Another important characteristic of blood perfusion technique specifically modified blood perfusion technique, which is the blood perfusion type selected for utilization with vascular networks, is that the transform does not alter the image characteristics. The transform is a point wise transform that keeps the structure of the image intact. It only adjusts the dynamic range and contrast. On the other hand, Pennes blood perfusion model differs from the other two perfusion models in this sense. Pennes blood perfusion model is not suitable for utilization with the vascular network method. The transform for Pennes model is not point wise. Upon visual inspection, the idea that the model is not suitable for utilization with vascular network method becomes evident with a resulting dotted image obtained from Pennes blood perfusion model. Segmenting the vascular network from such a dotted image is not feasible.

One other important characteristic of the blood perfusion is that the transform reduces in class differences. The transform does so while increasing the between class differences. This is a desired characteristic as well and aids the vascular network method for the purpose of classification. Recognition of a facial image is in fact finding the class that the facial image belongs to. This is done by utilizing between class differences. In class differences on the other hand, are differences 
between the images of the same person. Therefore, in class differences are not useful for the purpose of recognition.

The resulting image obtained from applying vascular network to blood perfusion transform of the thermal image also seems to produce same type of vascular network with the vascular network directly obtained from thermal image. Vascular networks obtained from thermal image and blood perfusion transform of the thermal image can be seen in Figure 4.10. The method is plausible theoretically and visual inspection supports the theoretical reasoning.

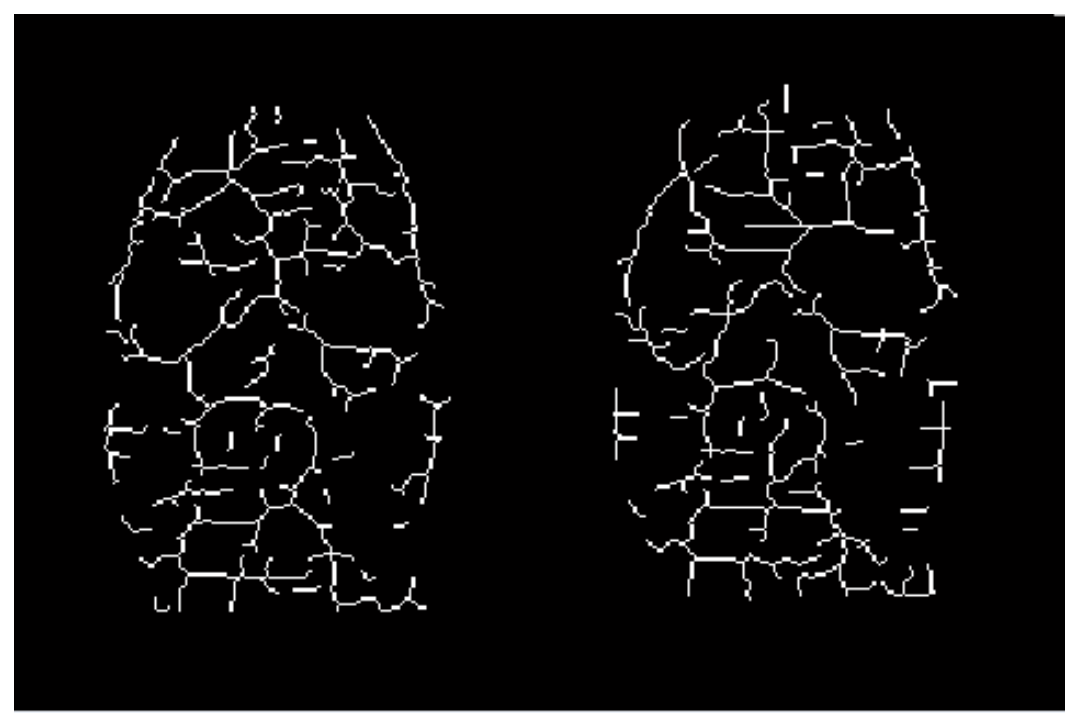

Figure 4.10 Vascular networks obtained from the same person utilizing thermal image (left) and blood perfusion transform of the thermal image (right)

When the proposed algorithm was tested the obtained results were encouraging. The application of vascular network method to perfusion transform of the thermal image led to significantly better results for both IRIS and Terravic databases. These results is presented in the following chapters in detail.

The proposed method starts with the application of modified blood perfusion transform. Blood perfusion transform of the test images are calculated first. Then the 
vascular network method described in previous method is followed step by step. First using morphological operations vascular network is obtained. Then post processing step is applied to this vascular network. The vascular network is thinned, short branches are removed and bifurcation points are obtained with the same procedure. For classification modified Hausdorff distance is utilized.

For our proposed method, 3 different feature vector approaches are investigated as in the previous vascular network method. These three feature vector approaches are exactly the same feature vector approaches that are presented in the vascular network method. The first (A) and second (B) feature vector approaches are introduced by Cho et al. [75]. The third feature vector approach (C) is also proposed in this thesis work for the first time. The first one (A) is the base method that utilizes a feature vector formed only by spatial $\mathrm{x}$ and $\mathrm{y}$ coordinates of bifurcation points. The second feature vector approach (B) utilizes a feature vector with four elements. These elements are spatial coordinates of bifurcation points, as in approach A, and the spatial coordinates of the center of gravity of the facial regions. In the third feature vector approach (C) is feature vectors are formed by adjusted bifurcation points. The gravity centers of two images are adjusted to coincide. The bifurcation points are also adjusted according to the difference between two gravity centers. The coinciding vascular networks are then compared for classification. We believe that this is the most robust way to compare two vascular networks for classification. 


\section{CHAPTER 5}

\section{EXPERIMENTAL RESULTS}

In this chapter implemented methods, which are explained thoroughly in chapter 4, are compared according to their recognition performances. Results are obtained from two separate available databases namely IRIS database and Terravic database.

\subsection{Databases}

Two databases are utilized in this thesis work, namely 'IRIS Thermal/Visible Face Database' and 'Terravic Facial IR Database'. Both databases are in LWIR band also called the thermal band.

'IRIS Thermal/Visible Face Database' is an available database and it contains 4228 images. The database contains images of 29 persons. The images presented in the database are indoor images. This database is very challenging for the purpose of face recognition. The database contains images that can test the limitations regarding face recognition for both modalities. It contains images for every person that have various rotations, changing illumination conditions, at least four different expressions, existence of glasses and hats which are challenging conditions for one or both of the modalities. In this thesis work infrared spectrum is concerned. For infrared band glasses are opaque so there are occlusions caused by the existence of glasses. Infrared spectrum images are not affected by changing illumination conditions as explained in the previous chapters. Expression changes and existence 
of disguise is still challenging for infrared spectrum images, although to a lesser degree compared to visible images. 203 images are selected to be utilized with the aforementioned 4 methods. That number represents 7 images per person. Some images and challenging conditions are given as an example in Figure 5.1.

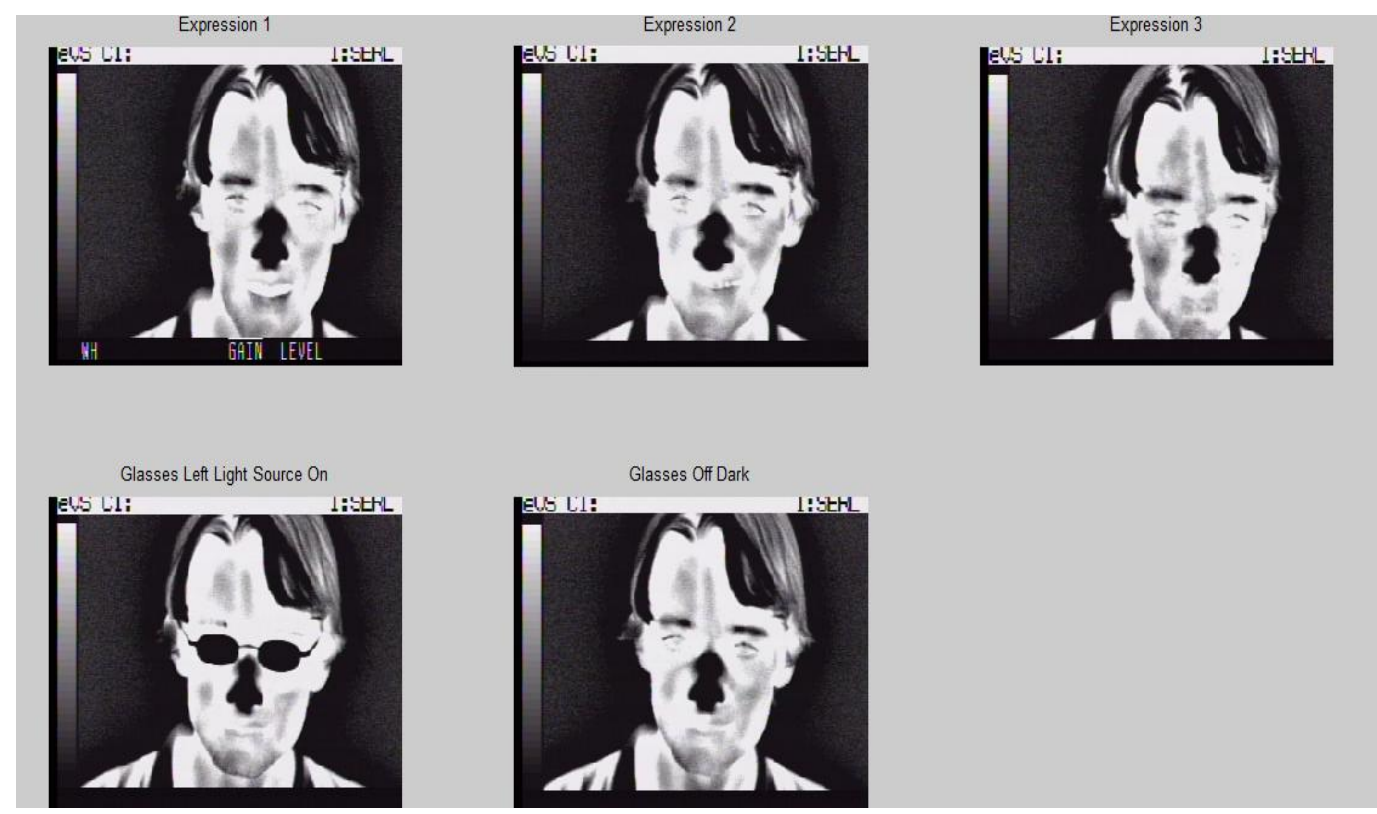

Figure 5.1 Various challenging conditions for images in IRIS database

“Terravic Facial IR Database' is the second database utilized in this thesis work. It consists of 24508 images that belongs to 20 persons. The database contains mostly indoor images. Some of the subjects' images are taken in outdoor conditions. Existence of glasses and hats, various rotations for every person present challenges for the purpose of face recognition. The illumination and expression conditions are not tested as in 'IRIS Thermal/Visible Face Database'. In 'IRIS Thermal/Visible Face Database' for every person there exist at least four different expressions and illumination changes such as light from left side, light from right side, light from both sides and no light. Those are not present in 'Terravic Facial IR Database'. Also the images in 'Terravic Facial IR Database' have less detail than the ones in 'IRIS Thermal/Visible Face Database'. There is a significant quality difference in images 
of both databases. For visual comparison an image from each database are given side by side in Figure 5.2. 270 images are selected to be utilized in the testing process of the aforementioned 4 methods. That number represents 15 images per person. Note that images that belong to 18 persons are utilized since the images that belong to the other two persons are corrupted in the downloaded database.
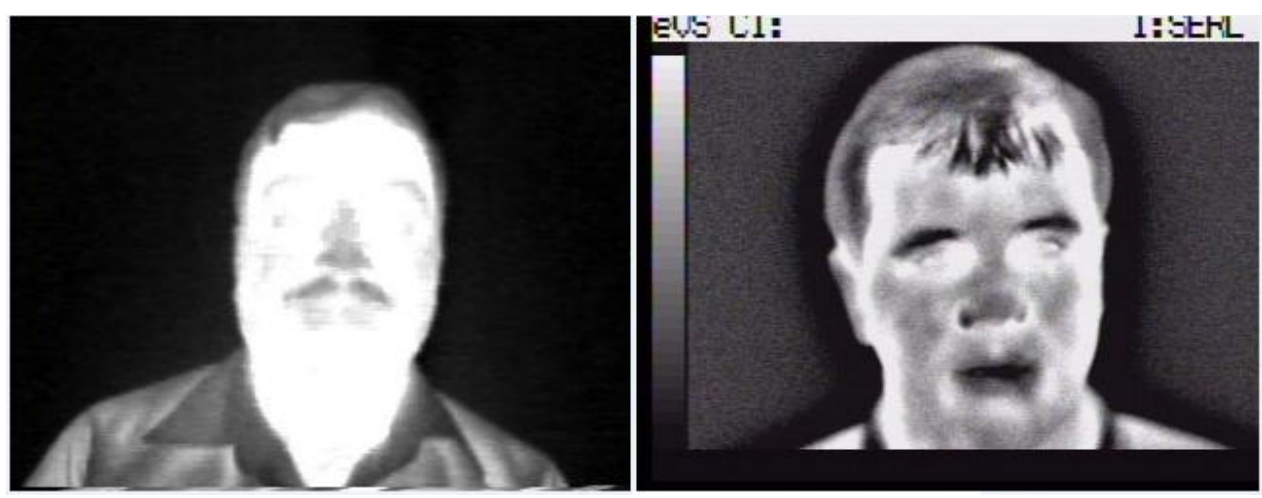

Figure 5.2 One image from each database are given side by side (Terravic image on the left side, IRIS image on the right side)

\subsection{Comparison Metrics}

Mainly utilized comparison metric in literature is rank-1 recognition rate. In this thesis work it is also the main comparison metric. It can be described as the probability that the recognized person by the algorithm is actually genuine person. In many papers it is the only metric presented in terms of accuracy.

False acceptance rate (FAR) and false rejection rate (FRR) are also presented for the implemented methods. In order to understand these error rates the definitions given below are required.

True Positive : The given test image is the person under consideration and also declared by the algorithm as that person. 
False Negative : The given test image is the person under consideration but declared by the algorithm as another person.

False Positive : The given test image is not the person under consideration but declared by the algorithm as that person.

True Negative : The given test image is not the person under consideration and also declared by the algorithm as another person.

True positive, true negative, false positive and false negative definitions can be summarized on the Table 5-1.

Table 5-1 Definitions of true positive, true negative, false positive and false negative

\begin{tabular}{|l|l|l|l|}
\cline { 3 - 4 } \multicolumn{2}{c|}{} & \multicolumn{2}{|l|}{ PREDICTION } \\
\cline { 3 - 4 } \multicolumn{2}{l|}{ MATCH } & \multicolumn{2}{l|}{ MISMATCH } \\
\hline \multirow{2}{*}{ ACTUAL } & MATCH & True Positive & False Negative \\
\cline { 2 - 4 } & MISMATCH & False Positive & True Negative \\
\hline
\end{tabular}

The FAR and FRR can be described as:

FAR is the probability that a non-matching person is defined wrongly as a matching person in the database. This rate is found as in Equation (5.1).

$$
F A R=\frac{F P}{F P+T N}
$$


FRR is the probability that a matching person is defined wrongly as a non-matching person in the database. This rate is found as in Equation (5.2).

$$
F R R=\frac{F N}{F N+T P}
$$

In our implementation the closest person is accepted as identified person. So for every test image one identified person is returned. It is either correct or wrong. That should be kept in mind when the error rates are evaluated.

\subsection{Results}

Results are presented in two sections divided according to the database that they are obtained. First the results for each method in the IRIS database are presented. In the second part, results for each method in Terravic database are presented.

\subsubsection{IRIS Database Results}

In this section, results obtained in IRIS database will be presented. Frontal images are utilized to obtain the following results. The focus was chosen as frontal images as they are the most challenging pose in infrared and visible spectrum face recognition. In literature algorithms report higher recognition rates for profile images. It is a result of the structure of the face. The frontal images do not give as much distinctive information as the profile images. Rank-1 recognition rates, error rates and computational time can be found in Table 5-2. 
Table 5-2: Results obtained for the implemented methods in IRIS Database

\begin{tabular}{|c|c|c|c|c|}
\hline Method & $\begin{array}{c}\text { Rank-1 } \\
\text { recognition rate }\end{array}$ & FAR & FRR & $\begin{array}{c}\text { Time } \\
\text { (seconds) }\end{array}$ \\
\hline PCA & $82.3 \%$ & 0.0633 & 0.177 & 0.16 \\
\hline Blood Perfusion + PCA & $84.2 \%$ & 0.0563 & 0.158 & 0.18 \\
\hline Vascular Network Method & $84.7 \%$ & 0.0545 & 0.153 & 1.05 \\
\hline Approach A & $99.5 \%$ & 0.00002 & 0.005 & 1.20 \\
\hline Vascular Network Method & $88.2 \%$ & 0.00422 & 0.118 & 1.13 \\
\hline Approach B & $96.5 \%$ & 0.00012 & 0.035 & 0.68 \\
\hline $\begin{array}{c}\text { Vascular Network Method } \\
\text { Approach C }\end{array}$ & $99.5 \%$ & 0.00002 & 0.005 & 0.80 \\
\hline $\begin{array}{c}\text { Blood Perfusion + Vascular } \\
\text { Network Method Approach A }\end{array}$ & $90.6 \%$ & 0.00334 & 0.094 & 0.812 \\
\hline $\begin{array}{c}\text { Blood Perfusion + Vascular } \\
\text { Network Method Approach B }\end{array}$ & $\begin{array}{c}\text { Blood Perfusion + Vascular } \\
\text { Network Method Approach C }\end{array}$ & & & \\
\hline
\end{tabular}

\subsubsection{PCA Method}

IRIS database contains challenging images as stated earlier. Early works that utilized PCA often presented high recognition rates in the high ninety percents. As an example Socolinsky et al. [26], [27], [28] and Selinger et al. [29] utilized PCA with LWIR images and reported results in the range of 95 percent. It is argued that the reason that these methods performs so well in infrared spectrum is the lack of 
challenging data for infrared spectrum. This proved to be the truth as later works reported much lower recognition rates. Buddharaju et al. [47] reported recognition rates ranging from $69.56 \%$ to $78.84 \%$ with images that have illumination and expression variances. In IRIS database there are also images for many subjects that contain glasses. This is another limitation of infrared spectrum. In IRIS database Yin et al. [78] employed PCA approach and reported recognition rates ranging from $51.61 \%$ with 5 dimensions to $83.21 \%$ with 30 dimensions. Our reported results are in the same range. It should be noted that the algorithm applied in PCA method is not the most sophisticated algorithm. PCA here is implemented to as the baseline algorithm. Also in this thesis work we are much more interested in the application of PCA with perfusion transform to observe whether utilizing perfusion transform improves the performance or not.

In our experimental works we obtained rank-1 recognition rate as $82.3 \%$. This is in the same range with the works in literature. The number of significant eigenvectors identified by Kaiser's rule is 27. PCA is a viable method that can be employed with infrared images.

\subsubsection{PCA Method with Blood Perfusion Transform}

In this method PCA is applied to the modified blood perfusion model data instead of directly applying it to thermal data. The obtained perfusion images and original thermal images from IRIS database can be seen in Figure 5.3, Figure 5.4 and Figure 5.5 . 


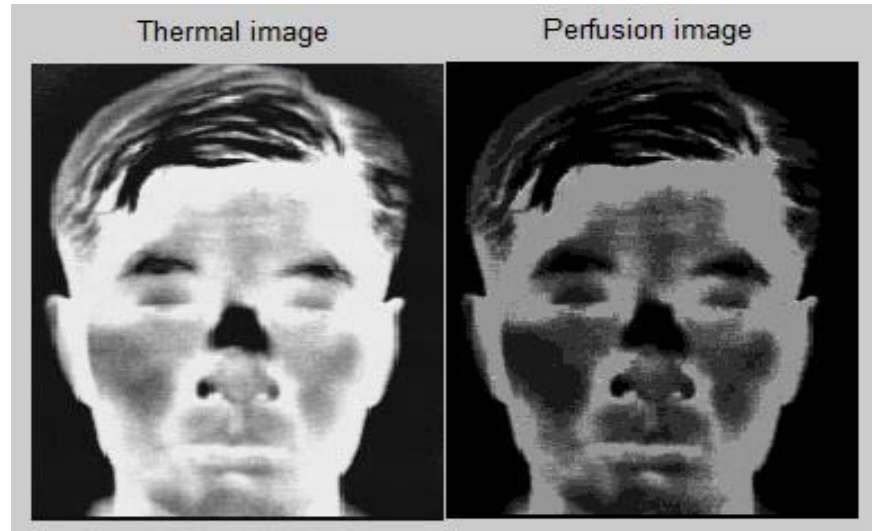

Figure 5.3 Original image and blood perfusion image

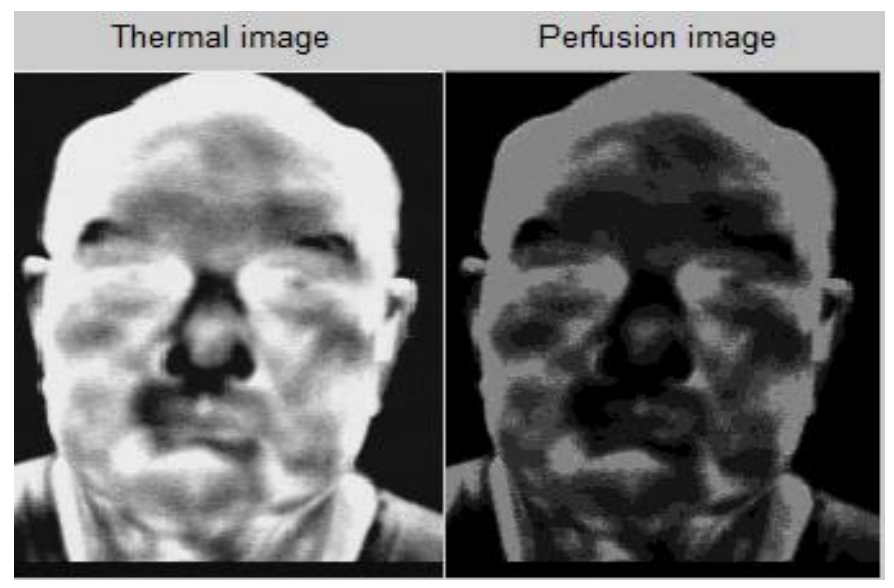

Figure 5.4 Thermal image and perfusion image

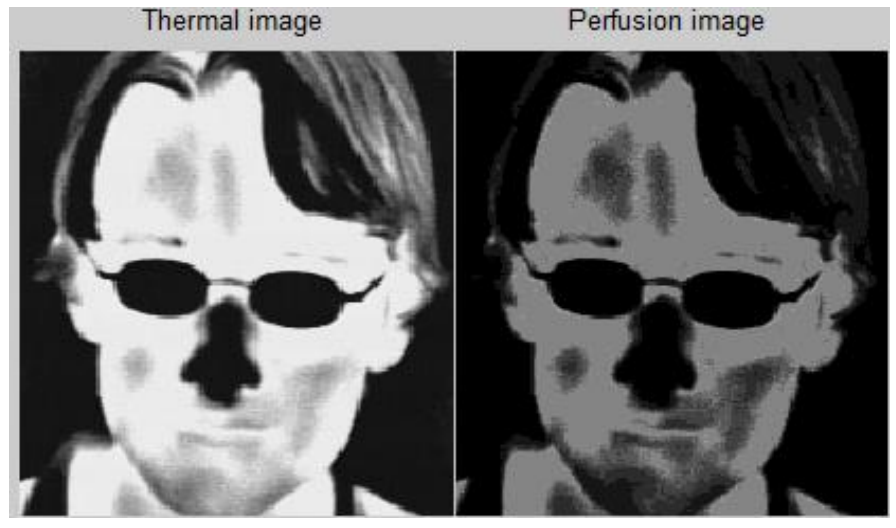

Figure 5.5 Thermal image and perfusion image 
In literature the works that presented a comparison between the perfusion model data and the thermal data for the same algorithm, reported better results for the perfusion model data. As an example Wu et al. [79] reported higher recognition rates for PCA with perfusion model data in their propriety database. PCA with thermal data recognition rate is $92.4 \%$ and with perfusion model data recognition rate is 94.4\%. This shows PCA is applicable to blood perfusion model data as well and in fact better results are obtained with the use of blood perfusion transform. The fact that PCA is applicable to perfusion model data is shown in many works that does not utilize PCA as classification scheme but utilize PCA as a space dimension reduction and representation tool before classification step. Xie et al. [80] utilized Fisher Linear Discrimination (FLD) Analysis to classify images. Before FLD PCA is employed for space dimension reduction. The recognition rate is $90.6 \%$ with thermal images and it is $97.8 \%$ with modified blood perfusion transform of the thermal images. $\mathrm{Wu}$ et al. [76] employed PCA and FLD as space dimension reduction tool and as classifier they utilized RBF neural network. Many different conditions are tested and in all of the tested conditions the use of perfusion transform of the thermal data outperformed the direct use of thermal data.

In our experimental work we obtained rank-1 recognition rate as $84.2 \%$. Our results comply with the results in the literature. Recognition rate is higher with blood perfusion transform of the thermal data than it is with thermal data. Recognition rate for thermal data was $82.2 \%$ as a reminder for the reader. Also FAR and FRR values are lower when the transform is used. Blood perfusion transform increases the contrast for high temperature regions and suppresses the contrast for low temperature regions. The variance in low temperature regions is high and they are usually caused by environmental effects. The variance in high temperature parts is low because of temperature regulation. So blood perfusion transform increases between class difference and decreases within class difference. Also blood perfusion transform of the thermal data is shown to be more robust than thermal data under varying ambient temperature, existence of glasses, the variance in thermal image 
caused by inhaling or exhaling, variance caused by metabolism and hair style in the work of Wu et al. [76]. Glasses, the effect of breathing patterns, different hair styles for the same person caused by imperfect segmentation are present in our tested database. These mentioned properties of blood perfusion transform improved the recognition rate of PCA, as expected.

\subsubsection{Vascular Network Method}

As stated earlier, three different feature vector approaches are investigated regarding this method. The approach referred as feature vector approach $\mathrm{A}$ is the base version proposed by Cho et al. [75]. In this feature vector approach feature vector only consists the coordinates of bifurcation points. The approach referred as feature vector approach $\mathrm{B}$ is the version which feature vectors are 4 dimensional. It is also proposed by Cho et al. [75]. Two dimensions are coordinates of bifurcation points, as in base version, and other two dimensions are the coordinates of the center of gravity of the facial region. The approach referred as feature vector approach $\mathrm{C}$ is proposed first time in this thesis work. In this feature vector approach the center of gravity of two vascular networks are adjusted to coincide. The bifurcation points are also adjusted according to the adjustment done for the gravity center. The idea was simple; the distances obtained when the centers are at the same point are more accurate than the distances obtained when the centers at two different positions.

The work proposed by Cho et al. [75] is a verification system. They did not report a recognition rate because of this fact. Error rates are reported as they are the meaningful metrics in verification systems. Buddharaju et al. [69] reported recognition rates ranging from $79.46 \%$ to $82.65 \%$ under different expression and illumination conditions in University of Notre Dame database. The vascular network is extracted in a very similar manner. The difference is in that work the ratio of number of matched branching points to the total number of branching points is utilized as the classifier. In this thesis modified Hausdorff distance is utilized. In 
their later work, Buddharaju et al. [71] improved the algorithm with a post processing step designed to remove fake branches and with the utilization of dual bootstrap iterative closest point algorithm as the new matching algorithm. The performance of the system increased and a maximum recognition rate of $88.87 \%$ is obtained. These two works utilize a threshold for the obtained score in order to declare a person recognized. The method proposed by Cho et al. [75] was selected because of the lower error rates.

Recognition rate for feature vector approach $\mathrm{A}$ is $84.7 \%$, for feature vector approach $\mathrm{B}$ it is $95.5 \%$ and for feature vector approach $\mathrm{C}$ it is $88.2 \%$. The recognition rates obtained for the vascular network method is comparable to the results obtained in the literature, if not higher. Proposed feature vector approach $\mathrm{C}$ performed better than feature vector approach $\mathrm{A}$ with respective recognition rates of $88.2 \%$ and $84.7 \%$. This is an expected result. In proposed feature vector approach $\mathrm{C}$ the distances obtained from coinciding vascular network offer more one to one correspondence between bifurcation points. The distance obtained between two points from two different vascular networks has a higher probability to be the actual corresponding distance. Feature vector approach $\mathrm{B}$ has the highest recognition rate with $99.5 \% .99 .5 \%$ recognition rate is caused by the difference in the coordinates of the gravity centers. The hausdorff distance of a recognized face is not a big number, most of the time smaller than 10 . The distance caused by the difference of the gravity centers dominates the overall result. Two images that belong to the same person with one image's center shifted a few pixels is most likely to be classified as different people. In order to test that a 4 pixel by 4 pixel shift is introduced. This may happen in a real world scenario with imperfect segmentation of the face or rotation. The recognition rate for feature vector approach B dropped from $99.5 \%$ to $83.3 \%$. Whereas the recognition rate for feature vector approach $\mathrm{C}$ dropped only from $88.2 \%$ to $83.3 \%$.

All of the recognition rates are higher compared to PCA methods given in previous sections. Also the error rates are lower than the PCA based methods. This is also an 
expected result. Vascular network employs physiological information which is more robust and does not change with varying conditions. The vascular network method is also more robust than PCA in the existence of glasses and expression changes. PCA representations change dramatically in those conditions but physiological information is not affected as dramatically as those representations. Vascular network is a more sophisticated algorithm that has more discriminative power. The feature based methods usually perform better in recognition task compared to holistic approaches and vascular network is one such method. In Figure 5.6 the steps of the algorithm and obtained vascular network can be seen.

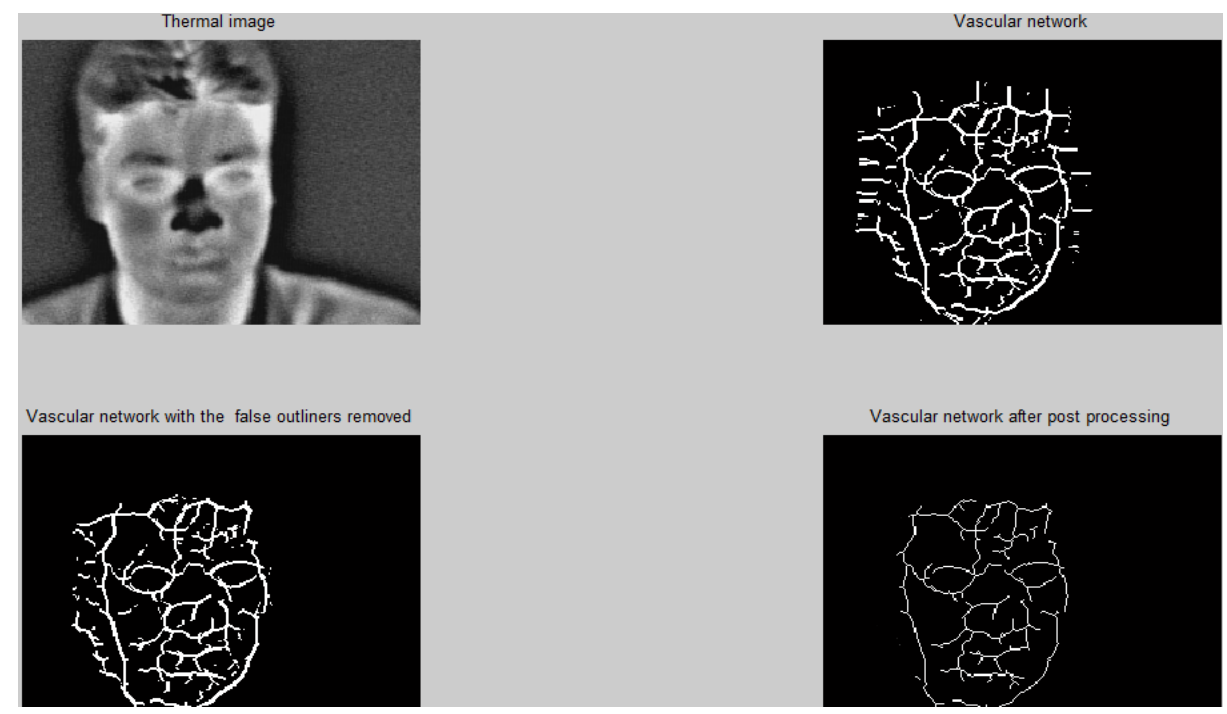

Figure 5.6 Steps of the algorithm and obtained vascular network

\subsubsection{Vascular Network Method Applied to Blood Perfusion Transform of the Thermal Data}

This method is introduced for the first time in this thesis work, to best of our knowledge. Utilization of the blood perfusion transform of thermal data leads to better performance for some methods compared to direct utilization of thermal data. The underlying idea for this method was to investigate whether this improvement is 
also present, if the blood perfusion transform of the thermal data is utilized with vascular network method. The rationale behind the method was thoroughly investigated in chapter 3 . The results obtained in IRIS database, actually in both databases, supported the idea of utilizing blood perfusion transform in conjunction with vascular network method. In IRIS database we obtained significantly better results with our proposed method for both feature vector approach A and feature vector approach $\mathrm{C}$. In the proposed method when feature vector approach B is utilized $99.5 \%$ recognition rate is obtained. When feature vector approach $\mathrm{A}$ is utilized in our proposed algorithm rank-1 recognition rate of $90.6 \%$ is obtained. Whereas the recognition rate for the same feature vector approach in vascular network method is $84.7 \%$. In our proposed algorithm when feature vector approach $\mathrm{C}$ is utilized, which is the feature vector approach proposed in this thesis work, the rank-1 recognition rate is $96.5 \%$. The corresponding recognition rate for feature vector approach $\mathrm{C}$ in vascular network method is $88.2 \%$. The feature vector approach $\mathrm{B}$ has the highest recognition rate. However, when a $4 \times 4$ shift is introduced to the center of gravity point the recognition rate for feature vector approach $\mathrm{C}$ decreases more than approach $\mathrm{B}$. The recognition rate drops from $99.5 \%$ to $95.1 \%$ for approach $\mathrm{C}$ and for approach B it drops from $96.5 \%$ to $95.1 \%$.

It is evident from the results that blood perfusion transform increases the performance of the vascular network method. The error rates also decrease with the use of blood perfusion transform. The steps of the proposed algorithm and obtained vascular network can be seen in Figure 5.7. 


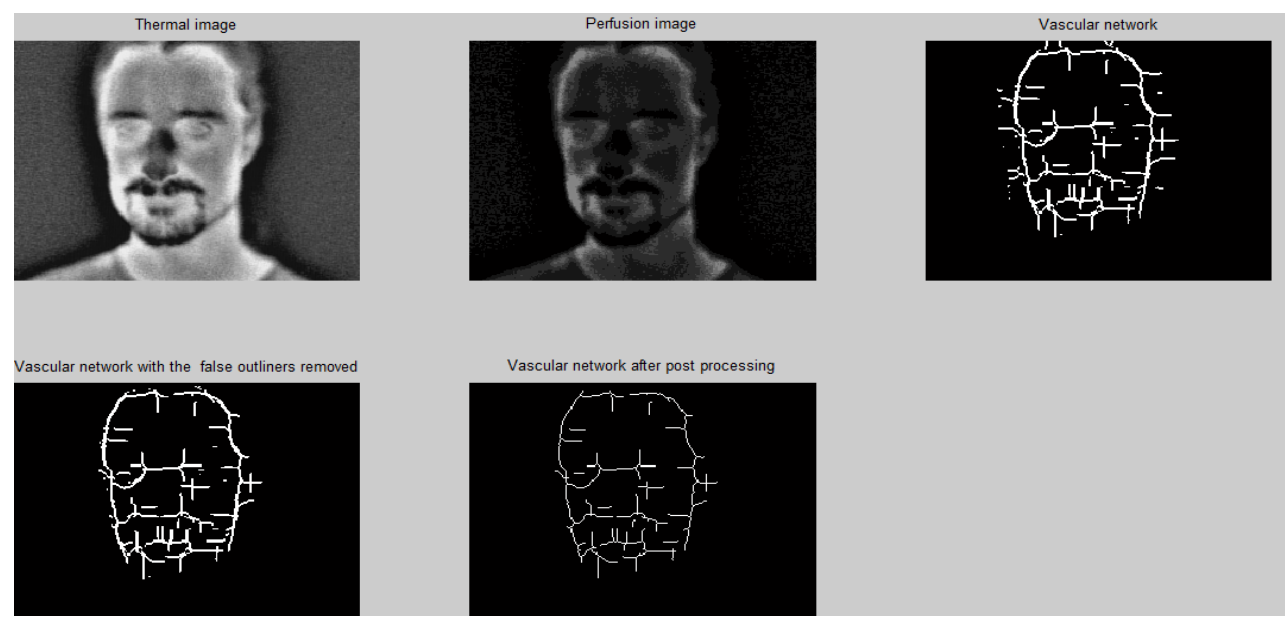

Figure 5.7 Steps of the proposed method and obtained vascular network

Another reason why proposed algorithm performs better than the vascular network method, other than the ones stated in chapter 3, may be that this proposed algorithm can be more successful in terms of reducing the fake branches in the vascular network. Fake may not be the most appropriate wording here but from the visual inspection and theoretical knowledge it is known that there are fewer branches in vascular networks obtained from the blood perfusion transform of the thermal data. Those branches may be the ones that are more significant, which leads to a better recognition performance. In literature fake branches are often cited as the main cause of degradation of performance.

Another observation made is that the difference between the second closest image and recognized image increases with the utilization of perfusion data. With thermal data for feature vector approach $\mathrm{C}$ the distance obtained for the second closest person is $10.8 \%$ higher than the distance obtained for the recognized person. With the use of blood perfusion transform, as proposed in this thesis work, that distance difference rose to $13.4 \%$. With less bifurcation points more distance between the recognized person and second closest person is obtained when the blood perfusion transform is used. This supports the idea that the proposed method has more 
discriminative power. Also it is a useful characteristic, if the algorithm is desired to be utilized with a threshold based recognition scheme.

\subsubsection{Terravic Database Results}

All of the aforementioned algorithms are tested in Terravic Database as well. Rank-1 recognition rates, error rates and computational time for frontal images can be found in Table 5-3.

Table 5-3 : Results obtained for the implemented methods in Terravic Database

\begin{tabular}{|c|c|c|c|c|}
\hline Method & $\begin{array}{c}\text { Rank-1 } \\
\text { recognition rate }\end{array}$ & FAR & FRR & $\begin{array}{c}\text { Time } \\
\text { (seconds) }\end{array}$ \\
\hline PCA & $77.8 \%$ & 0.0131 & 0.222 & 0.14 \\
\hline Blood Perfusion + PCA & $79.3 \%$ & 0.0122 & 0.207 & 0.15 \\
\hline Vascular Network Method & $82.2 \%$ & 0.0105 & 0.178 & 1.45 \\
\hline Approach A & $98.1 \%$ & 0.0011 & 0.019 & 1.31 \\
\hline $\begin{array}{c}\text { Vascular Network Method } \\
\text { Approach B }\end{array}$ & $90.0 \%$ & 0.0059 & 0.100 & 1.35 \\
\hline $\begin{array}{c}\text { Vascular Network Method } \\
\text { Approach C }\end{array}$ & $86.7 \%$ & 0.0078 & 0.133 & 1.33 \\
\hline $\begin{array}{c}\text { Blood Perfusion + Vascular } \\
\text { Network Method Approach A }\end{array}$ & $98.1 \%$ & 0.011 & 0.019 & 1.23 \\
\hline $\begin{array}{c}\text { Blood Perfusion + Vascular } \\
\text { Network Method Approach B }\end{array}$ & $91.1 \%$ & 0.052 & 0.089 & 1.31 \\
\hline $\begin{array}{c}\text { Blood Perfusion + Vascular } \\
\text { Network Method Approach C }\end{array}$ & 939 & & \\
\hline
\end{tabular}




\subsubsection{PCA Method}

Recognition rate for PCA method in the Terravic Database is $77.8 \%$. The number of significant eigenvectors decided by Kaiser's rule is 17 . There exist 18 different persons in Terravic database for our experimental work. The obtained recognition rate is lower than what is obtained in IRIS database. This was an anticipated result as the images in IRIS contain more detail. Those details offer a better PCA representation.

The obtained recognition rate is similar to what has been reported in the literature. Abas and Ono [81] compared their developed algorithm with eigenface method in Terravic database and the recognition rate they obtained for PCA was in the vicinity of $80 \%$. The number of eigenvectors or the algorithm for deciding significant eigenvectors are not stated. Also test to training image ratio is very low compared to the work in this thesis. The test to training ratio for their work is at most $4: 1$.

\subsubsection{PCA Method with Blood Perfusion Transform}

In this method PCA is applied to blood perfusion transform of the thermal data. Obtained rank-1 recognition rate is $79.3 \%$. PCA with blood perfusion transform of the thermal data performs better than PCA with thermal data. The comments made about the improvement of PCA with utilization of blood perfusion transform of the thermal data in IRIS database can be stated here as well. Images from Terravic database and corresponding perfusion images can be seen in Figure 5.8, Figure 5.9. 


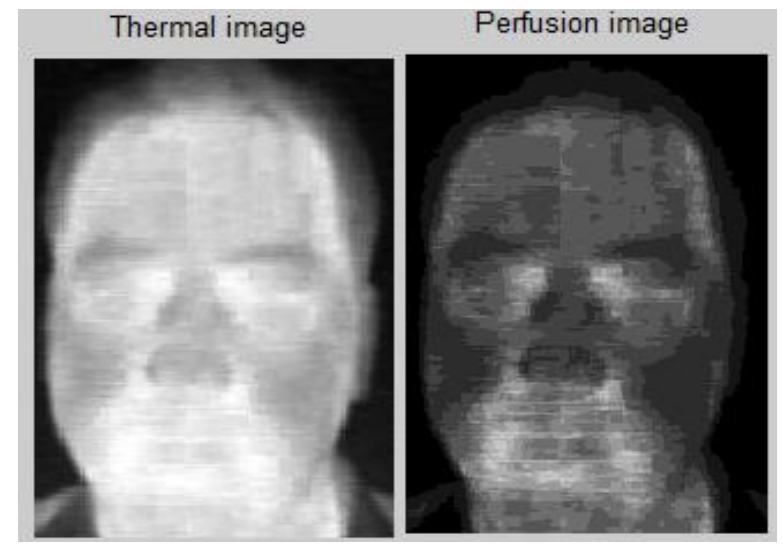

Figure 5.8 Thermal image and corresponding perfusion image

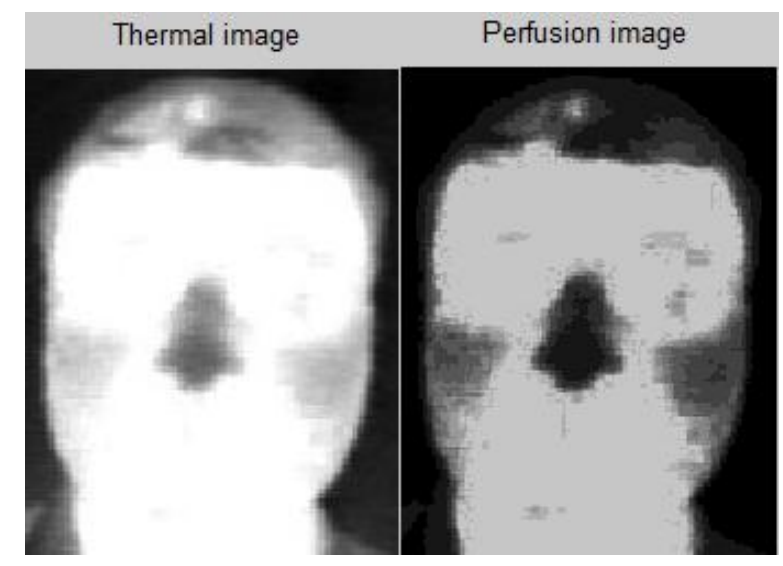

Figure 5.9 Thermal image and corresponding perfusion image

One important property of Terravic database is that it contains outdoor images for four subjects. The remaining subjects' images are indoor images. Blood perfusion method assumes indoor conditions. The ambient condition is assumed steady without wind and sun effect. Those cannot be guaranteed for outdoor images. That is the reason why the blood perfusion algorithm does not cause a significant improvement in performance. The improvement is more statistically significant in IRIS database, where all images are taken indoors. This effect can be seen even 
more clearly with the comparison of the results obtained in IRIS and Terravic databases for the next two methods.

\subsubsection{Vascular Network Method}

An image from the Terravic database, steps of the algorithm and resulting vascular network are illustrated in Figure 5.10.

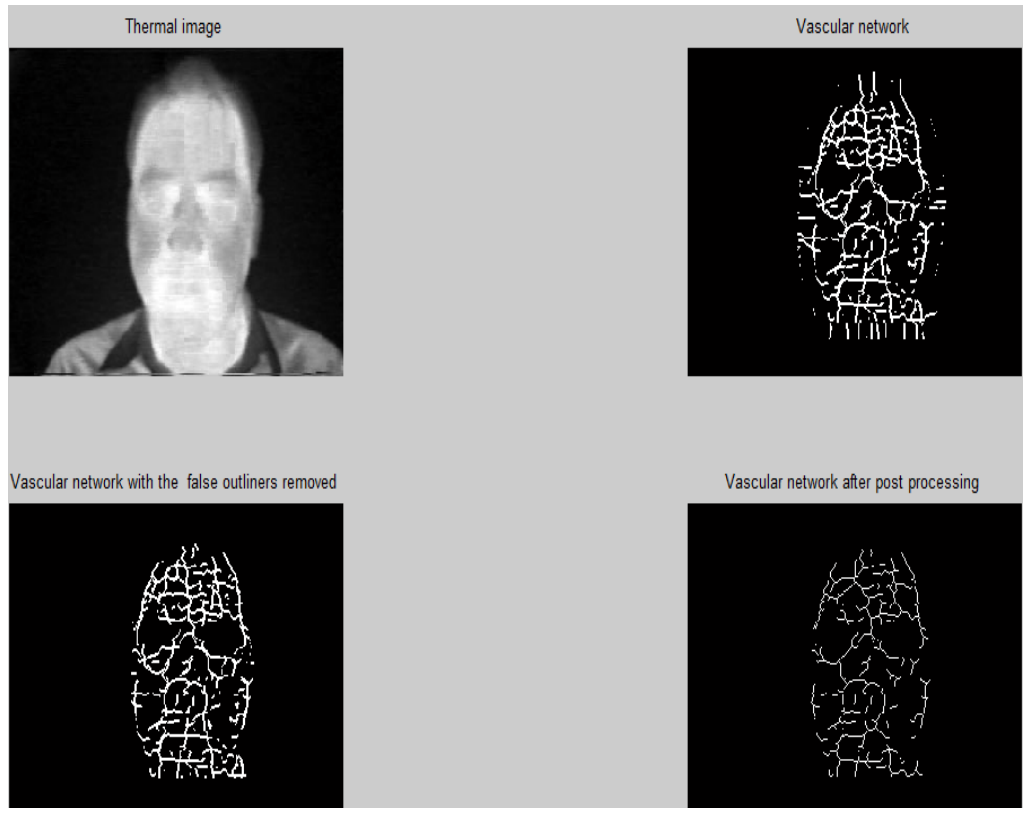

Figure 5.10 Steps of the vascular network method and obtained vascular network

Rank-1 recognition rates for vascular network method are given in Table 5-3. In Terravic database the best performing feature vector approach is approach $\mathrm{B}$ with recognition rate of $98.1 \%$, as the case in IRIS database. Feature vector approach $\mathrm{C}$ performs better than approach A with corresponding recognition rates of $90.0 \%$ and $82.2 \%$. When the $4 \times 4$ shift is introduced for the center of gravity the feature vector approach B fails again. The recognition rate for feature vector approach B decreases from $98.1 \%$ to $85.6 \%$. For approach $\mathrm{C}$ it only decreases from $90.0 \%$ to $87.4 \%$. 
All of the recognition rates are higher compared with the PCA methods given in previous sections. Also the error rates are lower. The comments made in IRIS database about this improvement also apply in Terravic database.

Comparing the results obtained in IRIS database with the ones in Terravic database, it is observed that Terravic database recognition rates are lower than IRIS database for vascular network. This was an anticipated result since the images in IRIS database contain more details and vascular network method utilizes these details. Vascular network approach utilizes small contrasts caused by the fact that the blood vessels are warmer than surrounding tissues. This small contrast is better detected in a higher quality image. The extraction of vascular network is a more accurate process in IRIS database. The vascular network method performs better with a better camera, thus better images.

\subsubsection{Vascular Network Method Applied to Blood Perfusion Transform of the Thermal Data}

An image from the Terravic database, steps of the proposed algorithm and resulting vascular network are illustrated in Figure 5.11. 


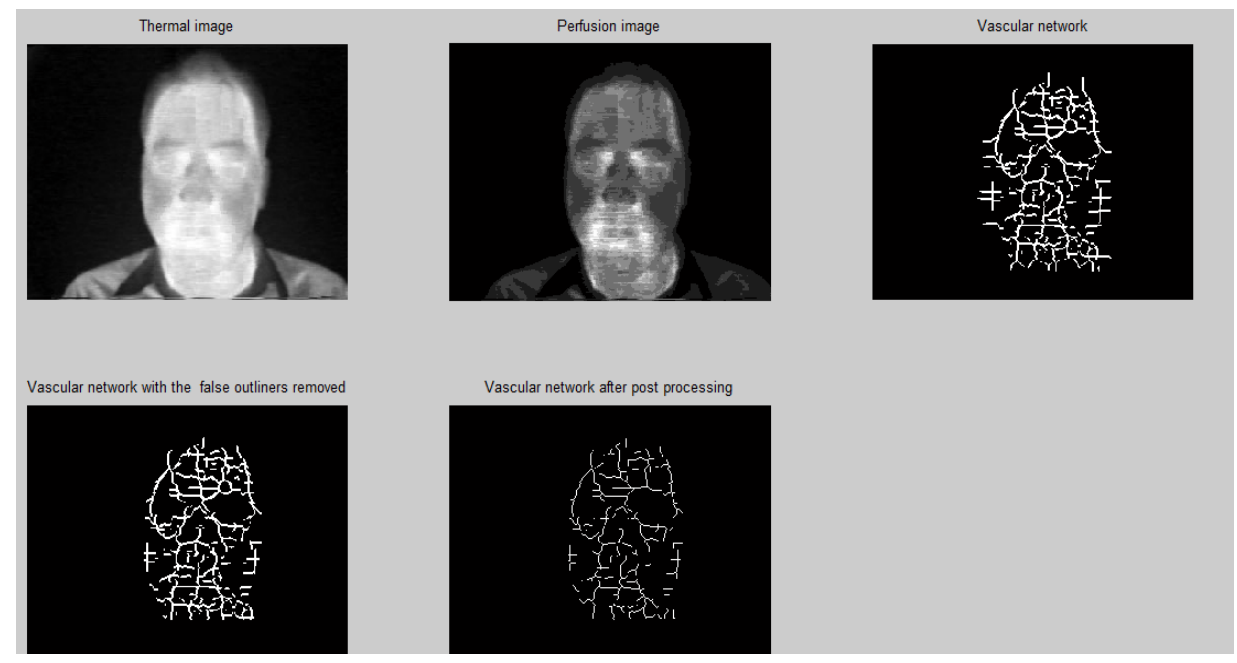

Figure 5.11 An image from the Terravic database, steps of the proposed method and obtained vascular network

Rank-1 recognition rates for vascular network method with blood perfusion transform of the thermal data are given in Table 5-3. Three feature vector approaches are investigated for the proposed method as well. In Terravic database the best performing feature vector approach is approach $\mathrm{B}$ with recognition rate of $98.1 \%$, as it is the case in IRIS database. Also feature vector approach C performs better than feature vector approach A with corresponding recognition rates of $91.1 \%$ and $86.7 \%$. Again when the $4 \times 4$ shift is introduced feature vector approach B fails. The recognition rate for feature vector approach B decreases from $98.1 \%$ to $90.0 \%$. Whereas the recognition rate for approach $\mathrm{C}$ only decreases from $91.1 \%$ to $90.0 \%$.

The proposed method is based on the utilization of blood perfusion transform with vascular network method. For all of the feature vector approaches better performance is obtained with the use of blood perfusion transform of the thermal data rather than direct use of the thermal data. This fact complies with the results obtained in IRIS database and supports the idea of utilizing blood perfusion transform with vascular network method. The distance difference between the recognized person and the second closest person increases with the use of blood 
perfusion transform. This suggests more discriminative power. For feature vector $\mathrm{C}$ without blood perfusion the distance for second closest person is $24.8 \%$ higher than the distance for the recognized person. That difference increases to $27.2 \%$ with the use of blood perfusion. As observed in IRIS database proposed algorithm offers more discriminative power with less bifurcation points.

As stated earlier the improvement with blood perfusion is less compared to IRIS database because of the outdoor images. Outdoor images degrade performance of blood perfusion. There is still an increase in performance, though; as majority of the images are still taken at indoor conditions. Also the recognition rates are not as high as they are for IRIS database, since images in Terravic database contain fewer details. 


\section{CHAPTER 6}

\section{CONCLUSION AND FUTURE WORK}

\subsection{Conclusion}

In this thesis, work, different methods for infrared, specifically LWIR, face recognition have been implemented and compared in terms of their recognition performances.

The methods are tested in IRIS and Terravic databases, both of which present challenging conditions for the recognition task.

The focus in infrared face recognition is shifted to methods that are designed specifically for infrared spectrum rather than applying visible spectrum algorithms to infrared images. Blood perfusion and vascular network are two such methods. In this thesis work it is illustrated that those methods can be utilized in conjunction.

Blood perfusion decreases within class variance and increases between class differences. Because of the nonlinear nature of blood perfusion, the high temperature regions are highlighted. Those regions are the regions that contain valuable information to classify a given person. Also the structure of a thermal facial image is not deformed with the blood perfusion transform. Those characteristics of blood perfusion transform make it useful for the purpose of face recognition. We have observed in this thesis that PCA and vascular network methods give better results 
with the utilization of the perfusion transform of the thermal data rather than the thermal data.

The vascular network approach with blood perfusion transform of the thermal data is proposed for the first in this thesis. The aforementioned characteristics of blood perfusion transform made the proposed method theoretically feasible and the results obtained from two databases comply with the theory. The proposed method performed best in both datasets. Proposed method has more discriminative power with less bifurcation points. Recognition rate of the proposed method is significantly high, over ninety percent. This suggests that the proposed method is a promising thermal face recognition technique and has good discriminative power. The vascular network based methods, both with thermal data and blood perfusion transform of the thermal data, outperformed PCA based methods.

In vascular network method and in the proposed method three different feature vector approaches are investigated. The base feature vector is formed by bifurcation points only. The second feature vector contains gravity center and bifurcation points. The third feature vector approach is also proposed for the first time in this thesis. In the proposed feature vector approach only bifurcation points are used but first two feature vectors are adjusted so that the centre of gravity points of both segmented faces are mapped to the same point. This feature vector approach leads to more accurate distances for classification and it is more robust to center of gravity shifts.

\subsection{Future Work}

The studied methods may be implemented with user defined thresholds as well. The classification decision in that implementation will be made with a comparison to the threshold. If the distance is below the threshold value, the person is indentified. Also the utilization of the proposed method as a verification system can be investigated. 
The pose estimation part can be made automatic as well. The images are first manually classified according to rotations. This task may be done automatically with rotation estimation. Further improvement can be made by compensating the deformation of vascular network caused by the rotation. Active appearance models can also be utilized to produce images with the exact rotation that the test image has. Ghiass et al. [73] utilizes a similar approach.

Another possible future work might be the extension of the proposed method to vesselness approach proposed by Ghiass et al. [72]. It could be investigated whether vesselness approach performs better with perfusion transform or not.

Bifurcation point concept of vascular network is based on fingerprint recognition. Some of the algorithms used in fingerprint recognition such as graph matching and distance ratios may also be applied to the vascular networks obtained from the face. This investigation may also be another possible future work. 


\section{REFERENCES}

[1] R. Bolle. Guide to biometrics. Springer Science \& Business Media, 2004.

[2] J.D. Woodward, N.M. Orlans, and P.T. Higgins. Biometrics: [identity assurance in the information age]. McGraw-Hill/Osborne New York, 2003.

[3] R. King, 'Biometric Research Note: Commercial Applications for Biometrics Growing', Biometric Update, 2012. [Online]. Available:

http://www.biometricupdate.com/201208/biometric-research-notecommercial-applications-for-biometrics-growing/. [Accessed:01- Feb- 2015].

[4] 'Global Biometric Systems Market Forecast \& Opportunities, 2018', TechSci Research, 2013.

[5] A. K. Jain, A. Ross and S. Prabhakar. An Introduction to Biometric Recognition. IEEE Trans. on Circuits and Systems for Video Technology, 14:4-20, 2004.

[6] W. Zhao, R. Chellappa, P. Phillips, A. Rosenfeld. Face recognition: A literature survey. ACM Computing Surveys (CSUR), 35(4):399-458, 2003.

[7] R.S. Ghiass, O. Arandjelović, A. Bendada and X. Maldague. Infrared face recognition: A comprehensive review of methodologies and databases. Pattern Recognition, 47(9):2807-2824, 2014.

[8] K. Bowyer, K. Chang and P. Flynn. A survey of approaches and challenges in 3D and multi-modal 3D+2D face recognition. Computer Vision and Image Understanding, 101(1):1-15, 2006.

[9] S.Z. Li and His Face Team, AuthenMetric F1: A Highly Accurate and Fast Face Recognition System.

[10] M.K. Bhowmik, A.N. Sarma, K. Saha, S. Majumder, G. Majumder, A. Saha, D. Bhattacharjee, D.K. Basu and M. Nasipuri. Thermal Infrared Face Recognition - A Biometric Identification Technique for Robust Security system, Reviews, Refinements and New Ideas in Face Recognition. INTECH Open Access Publisher, 2011.

[11] S.C. Liew. Electromagnetic Waves, Centre for Remote Imaging, Sensing and Processing.http://www.crisp.nus.edu.sg/research/tutorial/em.htm. [Accessed: 24- Feb- 2015].

[12] M. Akhloufi, A. Bendada and J. Batsale. State of the art in infrared face recognition. Quantitative InfraRed Thermography Journal, 5(1):3-26, 2008. 
[13] X. Zou, J. Kittler, and K. Messer. Face recognition using active near-ir illumination. In $B M V C$. Citeseer, 2005.

[14] L.B. Wolff, D.A. Socolinsky, and C.K. Eveland. Quantitative measurement of illumination invariance for face recognition using thermal infrared imagery. In International Symposium on Optical Science and Technology, pages 140-151. International Society for Optics and Photonics, 2003.

[15] G. Friedrich and Y. Yeshurun. Seeing people in the dark: Face recognition in infrared images. In Biologically motivated computer vision, pages 348-359. Springer, 2002.

[16] A. Persson and I. Buschmann. Vascular Growth in Health and Disease. Front. Mol. Neurosci., volume 4. 2011.

[17] I. Pavlidis, and P. Symosek. The imaging issue in an automatic face/disguise detection system. In Computer Vision Beyond the Visible Spectrum: Methods and Applications, 2000. Proceedings. IEEE workshop on, pages 1524, IEEE, 2000.

[18] F.J. Prokoski and R. Riedel. BIOMETRICS: Personal Identification in Networked Society, volume 479. Springer Science \& Business Media, 2006.

[19] D.A. Socolinsky, and A. Selinger, et al. Thermal face recognition in an operational scenario. In Proceedings of the 2004 IEEE Conference on Computer Vision and Pattern Recognition, volume 2, pages II-1012. IEEE,2004

[20] S.Q. Wu, W. Song, L.J. Jiang, S-L. Xie, F. Pan. W.Y. Yau, S. Ranganeth. Infrared face recognition by using blood perfusion data', In Audio- and Video-based Biometric Person Authentication, pages 320-328, 2005.

[21] S Wu, Z. Fang, Z. Xie, and W. Liang. Blood Perfusion Models for Infrared Face Recognition. InTech Open Access Publisher, 2008.

[22] S. Li, R. Chu, S. Liao and L. Zhang. Illumination Invariant Face Recognition Using Near-Infrared Images. Pattern Analysis and Machine Intelligence, IEEE Transactions on, 29(4): 627-639, 2007.

[23] W.F. Ganong, K.E. Barrett. Review of medical physiology, volume 21. McGraw-Hill Medical, 2005.

[24] R.G. Cutler. Face recognition using infrared images and eigenfaces, Citeseer, 1996.

[25] M. Turk, A.P. Pentland, et al. Face recognition using eigenfaces. In Computer Society Conference on Computer Vision and Pattern 
Recognition,1991. Proceedings CVPR'91, IEEE computer Science Conference on, pages 586-591.IEEE, 1991.

[26] D.A. Socolinsky, L.B. Wolff, J.D. Neuheisel, and C.K. Eveland. Illumination invariant face recognition using thermal infrared imagery. In Proc. IEEE Conference on Computer Vision and Pattern Recognition, pages 1-527, 2001.

[27] D. A. Socolinsky and A. Selinger. Thermal face recognition over time. In Proc. IAPR International Conference on Pattern Recognition, 2004, pp. 4187.

[28] D.A. Socolinsky, A. Selinger. A comparative analysis of face recognition performance with visible and thermal infrared imagery. Technical Report, DTIC Document, 2002.

[29] A. Selinger and D. Socolinsky. Appearance-based facial recognition using visible and thermal imagery: a comparative study. Technical Report, 2001.

[30] J. Kang, A. Borkar, A. Yeung, N. Nong, M. Smith, M. Hayes. Short wavelength infrared face recognition for personalization. In Image Processing, 2006 IEEE International Conference on (ICIP'06), pages 2757-2760, Oct 2006.

[31] X. Zou , J. Kittler, K. Messer. Face recognition using active Near-IR illumination. Proceedings of British Machine Vision Conference, pages 153$154,2005$.

[32] S. Zhao, R. Grigat. An automatic face recognition system in the near infrared spectrum. In Machine Learning and Data Mining in Pattern Recognition pages 437-444. Springer, 2005.

[33] T. Elguebaly and N. Bouguila. A Bayesian method for infrared face recognition. In Machine Vision Beyond Visible Spectrum, pages 123-138. Springer, 2011.

[34] Z. Lin, Z. Wenrui, S. Li, and F. Zhijun. Infrared face recognition based on compressive sensing and PCA. In Computer Science and Automation Engineering (CSAE), 2011 IEEE International Conference on, volume 2, pages 51-54. IEEE, 2011.

[35] Z. Pan, G. Healey, M. Prasad, and B. Tromberg. Multiband and spectral eigenfaces for face recognition in hyperspectral images. In Defense and Security, pages 144-151. International Society for Optics and Photonics, 2005. 
[36] Y. Yoshitomi, T. Miyaura, S. Tomita, and S. Kimura. Face identification using thermal image processing. In Robot and Human Communication, 1997. ROMAN'97, Proceedings., 6th IEEE International Workshop on, pages 374379. IEEE, 1997.

[37] G. Friedrich, Y. Yeshurun. Seeing people in the dark: face recognition in infrared images. In Proceedings of the International Workshop on Biologically Motivated Computer Vision (BMCV'02), volume 2525, pages 348-359, 2002.

[38] B. Abidi. Performance comparison of visual and thermal signatures for face recognition. In The Biometric Consortium Conference, 2003.

[39] S. Z. Li and his face team. AuthenMetric F1: A highly accurate and fast face recognition system. In Proc. IEEE International Conference on Computer Vision-Demos, 2005.

[40] S. Li, R. Chu, M. Ao, L. Zhang, and R. He. Highly accurate and fast face recognition using near infrared images. In Advances in Biometrics, pages 151-158. Springer, 2006.

[41] S. Li, L. Zhang, S. Liao, X. Zhu, R. Chu, M. Ao, and R. He. A near-infrared image based face recognition system. In Proc. IEEE International Conference on Automatic Face and Gesture Recognition, pages 455-460. IEEE, 2006.

[42] D. Goswami, C. H. Chan, D. Windridge, and J. Kittler. Evaluation of face recognition system in heterogeneous environments (visible vs NIR). In Computer Vision Workshops(ICCV Workshops), 2011 IEEE International Conference on, pages 2160-2167. IEEE, 2011.

[43] A. Srivastava, X. Liu, B. Thomasson, and C. Hesher. Spectral probability models for infrared images and their applications to ir face recognition. CVBVS, 2001.

[44] A. Srivastana and X. Liu. Statistical hypothesis pruning for recognizing faces from infrared images. Image and Vision Computing, 21(7): pp. 651$661,2003$.

[45] F. Nicolo and N. A. Schmid. A method for robust multispectral face recognition. in Image Analysis and Recognition, pages 180-190. Springer, 2011.

[46] Z. Xie, S.Wu, G. Liu, and Z. Fang. Infrared face recognition based on radiant energy and curvelet transformation. In Information Assurance and Security (IAS), 2009. Fifth International Conference on, volume 2, pages 215-218. IEEE, 2009. 
[47] P. Buddharaju, I. T. Pavlidis, and P. Tsiamyrtzis. Physiology-based face recognition. In Advanced Video and Singal Based Surveillance, 2005. AVSS 2005, IEEE Conference on, pages 354-359. IEEE, 2005.

[48] X. Chen, P. Flynn, K. Bowyer. PCA-based face recognition in infrared imagery: baseline and comparative studies. In Analysis and Modeling of Faces and Gestures 2003. AMFG 2003. IEEE International Workshop on, pages 127-134. IEEE, 2003.

[49] X. Chen, P. Flynn, K. Bowyer. IR and visible light face recognition. Computer Vision \&Image Understanding, volume 99, pages 332-358, 2005.

[50] X. Chen, Z. Jing, G. Xiao. Fuzzy fusion for face recognition. In Proceedings of the International Conference on Fuzzy Systems and Knowledge Discovery (FSKD'05), pages 672-675, 2005.

[51] P. Buddharaju, I. Pavlidis. Multi-spectral face recognition fusion of visual imagery with physiological information. Face Biometrics for Personal Identification, Signals and Communication Technology, pages 91-108. Springer, 2007.

[52] K. Bowyer, K. Chang, P. Flynn, X. Chen. Face recognition using 2-d, 3-d, and infrared: is multimodal better than multisample?. In Proceedings of the IEEE, vol. 94(11):2000-2012, 2006.

[53] I. Kakadiaris, G. Passalis, T. Theoharis, G. Toderici, I. Konstantinidis, Murtuza N. Multimodal face recognition: combination of geometry with physiological information. In Computer Vision and Pattern Recognition, 2005. CVPR 2005. IEEE Computer Society Conference on, pages 1022-1029. IEEE 2005.

[54] I. Kakadiaris, G. Passalis, T. Theoharis, G. Toderici, I. Konstantinidis, N. Murtuza. 8DTHERMO CAM: combination of geometry with physiological information for face recognition. In Computer Vision and Pattern Recognition, 2005. CVPR 2005. IEEE Computer Society Conference on, volume 2, pages 1183. IEEE, 2005.

[55] A. Gyaourova, G. Bebis, I. Pavlidis. Fusion of infrared and visible images for face recognition. Proceedings of the European Conference on Computer Vision (ECCV'04), volume 4, pages 456-468, 2004.

[56] S. Singh, A. Gyaourova, G. Bebis, I. Pavlidis. Infrared and visible image fusion for face recognition. Proceedings of the SPIE: Biometric Technology For Human Identification, volume 5404, pages 585-596, 2004.

[57] R. Singh, M. Vatsa, A. Noore. Hierarchical fusion of multi-spectral face images for improved recognition performance. Information Fusion, In Press, 2007. 
[58] G. Bebis, A. Gyaourova, S. Singh, I. Pavlidis. Face recognition by fusing thermal infrared and visible imagery. Image \& Vision Computing, volume 24, pages 727-742, 2006.

[59] H. Hariharan, A. Koschan, B. Abidi, A. Gribok, M. Abidi. Fusion of visible and infrared images using empirical mode decomposition to improve face recognition. in Image Processing (ICIP'06), IEEE International Conference on, pages 2049-2052, 2006.

[60] J. Heo. Fusion of visual and thermal face recognition techniques: a comparative study. Master Thesis, The University of Tennessee, Knoxville 2003.

[61] O. Arandjelovic, R. Hammoud, R. Cipolla. On person authentication by fusing visual and thermal face biometrics in Video and Signal Based Surveillance, 2006. AVSS'06, IEEE International Conference on 2006, pages 50-50. IEEE, 2006.

[62] T. Bourlai, N. Kalka, A. Ross, B. Cukic, L. Hornak. Cross-spectral face verification in the short wave infrared (SWIR) band. In Pattern Recognition IAPR,2010 20th International Conference on, pages 1343-1347, 2010.

[63] B. Klare and A. K. Jain. Dynamic scene shape reconstruction using a single structured light pattern. In Pattern Recognition IAPR, International Conference on, pages 1513-1516, 2008.

[64] T. Bourlai, A. Ross, C. Chen, and L. Hornak. A study on using mid-wave infrared images for face recognition. In SPIE Defense Security and Sensing, 2012.

[65] S. Filipe, L.A. Alexandre. Thermal Infrared Face Segmentation: A New Pose Invariant Method. 6th Iberian Conference on Pattern Recognition and Image Analysis, IbPRIA, pages 632-639. Springer, 2013.

[66] T. F. Chan, L.A. Vese. Active contours without edges. IEEE Transactions on Image Processing, 10(2):266-277, 2001.

[67] M. Eryilmaz. Face segmentation in thermal images. Master Thesis, Middle East Technical University, Ankara, 2015.

[68] E.J. Candes, X. Li, Y. Ma, J. Wright. Robust Principal Component Analysis?. Journal of ACM (JACM), 58(3):11, 2011.

[69] P. Buddharaju, I. T. Pavlidis, P. Tsiamyrtzis, M. Bazakos. Physiology-based face recognition in the thermal infrared spectrum. In Pattern Analysis and Machine Intelligence, IEEE Transactions on, 29(4):613-626. IEEE, 2007. 
[70] Y. Pinar and F. Govsa. Anatomy of the superficial temporal artery and its branches: its importance for surgery. Surgical and Radiologic Anatomy, 28(3):248-253, 2006.

[71] P. Buddharaju and I. Pavlidis. Physiological face recognition is coming of age. In Computer Vision and Pattern Recognition, 2009. CVV) 2009. IEEE Conference on, pages 128-135, June 2009.

[72] R. S. Ghiass, O. Arandjelovi_c, A. Bendada, X. Maldague. Vesselness features and the inverse compositional AAM for robust face recognition using thermal IR. CoRR, abs\1306,1609, 2013.

[73] R. S. Ghiass, O. Arandjelovi_c, A. Bendada, X. Maldague. Illuminationinvariant face recognition from a single image across extreme pose using a dual dimension AAM ensemble in the thermal infrared spectrum. CoRR, abs $\backslash 1306,1822,2013$.

[74] A.F. Frangi, W.J. Niessen, K.L. Vincken, M.A. Viergever. Multiscale vessel enhancement filtering. Medical Image Computing and Computer-Assisted Intervention- MICCAI'98, pages 130-137, 1998.

[75] S. Cho, L. Wang, and W. J. Ong. Thermal imprint feature analysis for face recognition. in ISIE, pages 1875-1880, 2009.

[76] S. Wu, Z. Gu, K.A. Chia, S.H. Ong. Infrared Facial Recognition Using Modified Blood Perfusion. In Information, Communications \& Signal Processing, 6th International Conference on, pages 1-5, Dec 2007.

[77] Z. Xie, S. Wu, C He, Z. Fang, J. Yang. Infrared face recognition based on blood perfusion using bio-heat transfer model. In $C C P R$, pages 1-4, 2010.

[78] H.F. Yin, X.J. Wu, X.Q. Sun. Client Specific Image Gradient Orientation for Unimodal and Multimodal Face Representation. Multimodal Pattern Recognition of Social Signals in Human-Computer-Interaction, pages 15-25. Springer International Publishing, 2015.

[79] S. Q. Wu, L. Z. Wei, Z. J. Fang, R. W. Li, X. Q. Ye. Infrared face recognition based on blood perfusion and sub-block DCT in wavelet domain. in Wavelet Analysis and Pattern Recognition, 2007. ICWAPR'07. International Conference on, volume 3, pages 1252-1256. IEEE, 2007.

[80] Z. Xie, G. Liu, S. Wu, Z. Fang. Infrared face recognition based on blood perfusion and fisher linear discrimination analysis. pages 85-88, 2009.

[81] K.H. Abas, O. Ono. Multilayer Infrared-Based Face Identification System for Security Vehicle-Robot Vision. International Journal of Simulation, Systems, Science \& Techno, 11(5), 2010. 